UNDERSTANDING TEACHER PERSPECTIVES ABOUT INSTRUCTIONAL TECHNOLOGY FROM AN ECOLOGY OF RESOURCES FRAMEWORK

\author{
A Dissertation \\ presented to \\ the Faculty of the Graduate School \\ at the University of Missouri-Columbia \\ In Partial Fulfillment \\ of the Requirements for the Degree \\ Doctorate of Education \\ by \\ CHRISTINE KRAFT DIGGS \\ Dr. Sarah Diem, Dissertation Supervisor \\ DECEMBER 2014
}


(C) Copyright by Christine Kraft Diggs, 2014 All Rights Reserved 
The undersigned, appointed by the Dean of the Graduate Faculty, have examined the dissertation entitled:

\section{Understanding Teacher Perspectives about Instructional Technology from an Ecology of Resources Framework}

presented by Christine Kraft Diggs, A candidate for the degree of doctor of education, and hereby certify that, in their opinion, it is worthy of acceptance.

Dr. Sarah Diem, Advisor

Dr. Noelle Arnold

Dr. Julie Caplow

Dr. M. Carol Maher 


\section{ACKNOWLEDGEMENTS}

I would like to express my deep appreciation and gratitude to my advisor, Dr. Sarah Diem, for the patient guidance and mentorship she provided to me. Her assistance was invaluable to my success in completing this research, and her excellent feedback greatly improved its quality. I would also like to thank my committee members, Drs. Noelle Arnold, Julie Caplow, and Carol Maher for the friendly guidance and thought-provoking suggestions they offered me.

I would also like to thank my professors and fellow doctoral students for their support, feedback, and friendship, especially the "Berg” group, whose stimulating conversation and shared laughter enriched my educational journey. I would also like to express my sincere appreciation for the teachers and administrators who willingly shared their time and experiences with me. It was a true pleasure spending time at their school and in their classrooms.

I would like to thank my staff and friends who supported and encouraged me over the years. I would especially like to thank my sister Carolyn for her weekly "pep emails” while I was writing this dissertation. And to all of my brothers, sisters and daughters, thank you for believing in me. Finally, I must acknowledge the innumerable sacrifices made by my husband, Craig, who lovingly took care of our household while I pursued this degree. This achievement would not have been possible without Craig's unwavering patience and support. 


\section{TABLE OF CONTENTS}

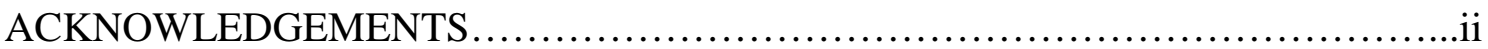

LIST OF FIGURES.................................................................

LIST OF TABLES..............................................................

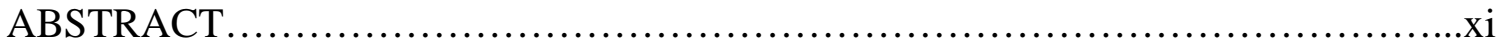

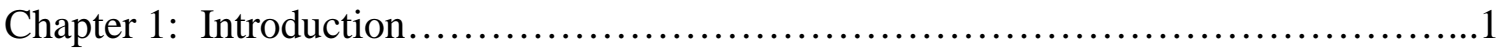

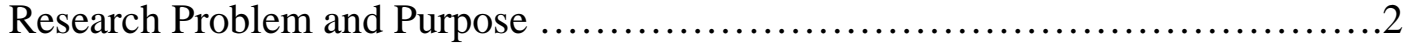

Research Questions......................................................4

Conceptual Framework.....................................................4

Positionality.................................................................

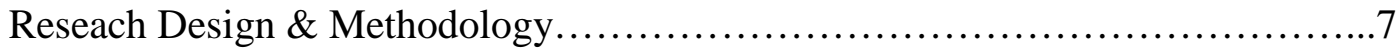

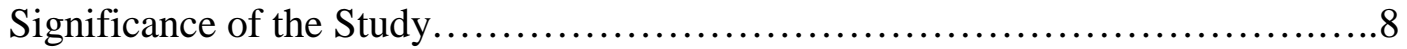

Definition of Key Terms................................................9

Conclusion............................................................... 10

Chapter 2: Literature Review.................................................12

Educational Technology during the Past 20 Years............................13

The rise of the Internet...........................................13

Benefits and perils of the Internet..........................14

Move from device centric focus to curriculum centric focus.............15

Technology as a tool for learning................................16

Global impact of technology $\ldots \ldots \ldots \ldots \ldots \ldots \ldots \ldots \ldots \ldots \ldots \ldots \ldots \ldots \ldots$

Implementing Innovations and its Related Challenges.......................20

Diffusion of Innovations.......................................20 
Disruptive innovations. .22

The role of internal dissonance........................22

The role of perceived usability......................22

Importance of shared vision.........................23

The role of opinion leaders............................24

The role of motivation...............................25

The role of the environment...........................25

Implementing educational innovation.................................26

Adult Learning as a Vehicle for Change....................................27

Learning and change...........................................27

Adult learning............................................28

Motivation for learning.................................28

Reflection and learning..................................29

The role of experience in learning..........................30

Pedagogy and change...................................30

Exploring Instructional Technology Use Through Sociocultural Learning

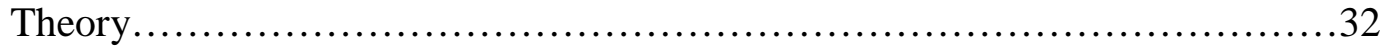

The social nature of learning....................................33

The Zone of Proximal Development................................33

Mediation and internalization......................................34

Situational influences.......................................35

Social capital..........................................35

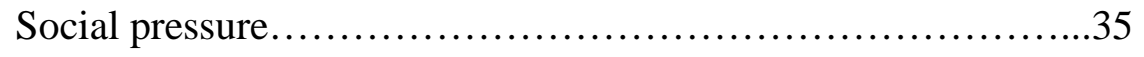

Degree of trust..........................................36 
Personal influences.........................................36

Affective factors..........................................37

Cognitive factors.......................................37

Risk perceptions........................................38

The Learner Centric Ecology of Resources Framework..................39

Teachers situated as learners in the center of the context.........40

Context as consisting of four relational dimensions of resources..41

Filters affecting the teacher and the four dimensions............43

Filters affecting the people dimension...................43

Filters affecting the tools dimension...................44

Filters affecting the knowledge and skills dimension......44

Filters affecting the environment dimension.............45

Studying filters affecting technology use...............46

Complex interactions among dimensions and learners............47

Summary................................................49

Chapter 3: Research Design and Methodology.................................52

Purpose of the Study................................................53

Research Questions...................................................54

Research Design....................................................54

Participants and Setting...............................................56

Data Collection........................................................58

Data collection tools...........................................58

Interviews..............................................59 


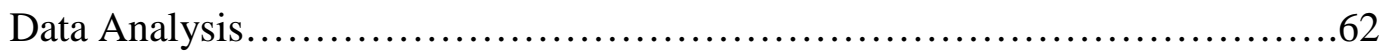

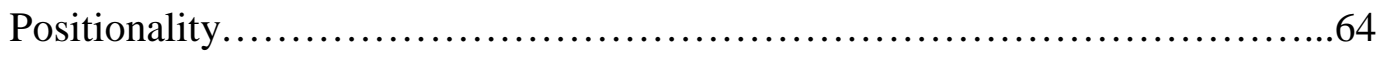

Human Subjects' protection and other ethical considerations....................65

Trustworthiness..........................................................66

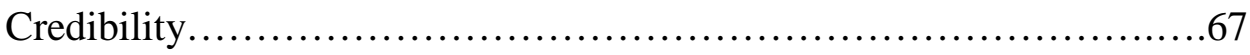

Dependability ................................................67

Transferability..................................................68

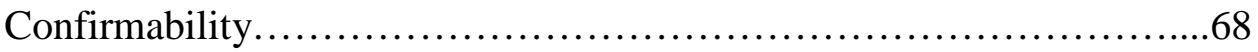

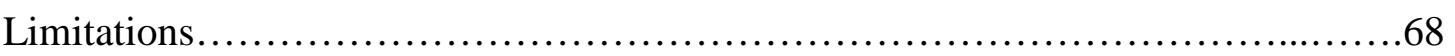

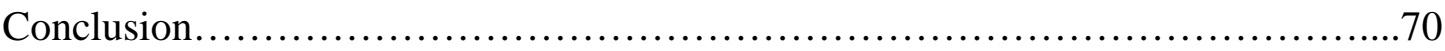

Chapter 4: Findings................................................................

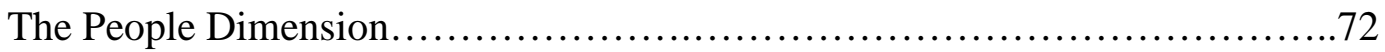

Technical support staff members are helpful............................72

Colleagues and students help with strategies............................74

Collaboration among teachers facilitate successful laptop use.............76

Expectations are implied through provision of resources..................80

Teachers appreciate leaders respect for their judgment...........83

Professional Learning Teams facilitate laptop use.........................84

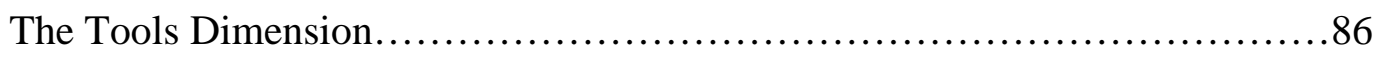

Laptops improve delivery of instruction............................... 86

Laptops eliminate need for scheduling access to computers................90

Laptops facilitate inquiry based, student-centered instruction..............91 
Effective instruction is facilitated by print and electronic tools

Effective instruction sometimes requires devices for each student..94

Availability of multiple technology tools enables active learning........95

Students with no Internet access or devices at home..................98

Software and device compatibility challenges.......................99

The Knowledge and Skills Dimension................................101

Knowledge about modeling approach facilitates laptop use............104

Teacher knowledge is sufficient but they are open to more learning.....105

The Environment Dimension.............................................107

Professional Learning Team time enables collaboration and learning....107

Block scheduling facilitates learning and laptop use..................110

School culture represents sharing, diversity and academic excellence.....111

Professional development sessions encourage learning and sharing........113

The Teacher as Learner...............................................115

Teachers want to improve and challenge themselves..................115

Teachers want to improve instruction for their students................116

Learning by doing............................................118

Learning through collaboration and observation.......................119

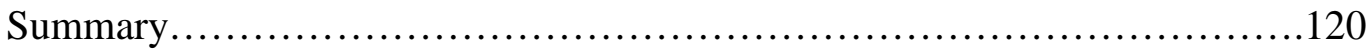

Chapter 5: Discussion, Conclusions and Implications...........................123

Discussion.............................................................

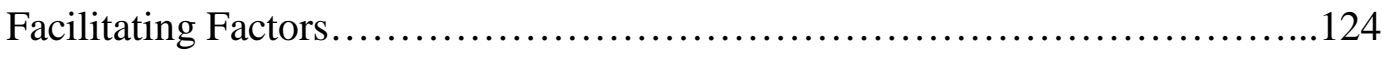

Professional Learning Teams (PLTs) ............................124 
Value of Peer Relationships

Technology as a Tool for Learning..................................127

The Eductional Context.............................................128

The Role of Explicit Expectations.................................129

Barrier Factors....................................................130

The Digital Divide.............................................130

Issues of Connectivity and Compatibility.........................131

Conclusions........................................................132

Implications for Future Research and Practice...........................135

Summary .........................................................138

Appendix A: Teacher Interview Protocol................................140

Appendix B: Classroom Observation Guide..............................142

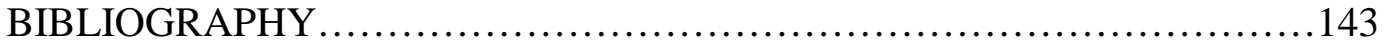

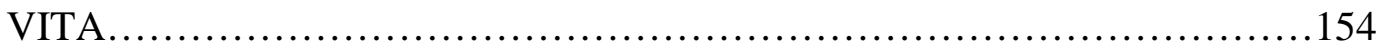




\section{LIST OF FIGURES}

Figure 1. Sociocultural Learning and the Ecology of Resources Framework.......48 


\section{LIST OF TABLES}

Table 1. Comparison of Student Test Scores of Modeling vs Traditional Instruction.........80 


\begin{abstract}
Technology spending for our K-12 schools continues to increase as schools transition from an emphasis on print resources towards an increasing amount of digital resources. Despite the permeation of technology in education, instructional use of technology by teachers remains sporadic. This dissertation reports on an exploratory, single case study which examined seven high school science teachers' views on the factors affecting their instructional use of technology, and their process of learning as adults.
\end{abstract}

Using the holistic ecology of resources framework combined with sociocultural learning theory, this study situated teachers as learners at the center of their educational context in order to explore their perspectives about the laptops they received in place of new textbooks. Interviews and classroom observations were used to investigate the convergence of teachers’ personal and situational influences affecting their use of instructional technology tools. This study identifies facilitative and barrier factors affecting teacher learning and their use of technology in their classrooms.

Findings show professional learning teams (PLTs) were the primary facilitating factor enabling teacher collaboration and learning as they used the laptops to enhance implementation of their science curriculum. Findings also show barriers exist in relation to connectivity and compatibility issues and the digital divide. This research finds it requires the whole school community working as an integrated system, rather than in isolation, for teachers to successfully use technology tools to reach and engage students in their $21^{\text {st }}$ century environment. 


\section{Chapter 1: Introduction}

Educational technology spending averages 56 billion dollars per year for our nation's schools (Johnson, 2012). Over the course of the past 20 years, emphasis on technology in education has grown significantly, moving from having a device-centric focus to a curriculumcentric one (Diem, 2010; Fullan, 2012). Despite the permeation of technology in every part of our society, instructional use of technology by teachers remains sporadic. A recent large international study on technology in education found a deficit exists between the rich digital environment students inhabit and their educational world, where teachers have been slow to embrace technology. Despite school districts' significant investments in educational technology, teachers continue to exhibit a reluctance to embrace the use of technology resources in their teaching (Luckin et al., 2012). Many current studies focused on technology’s impact on learning investigate how technology supports current instructional practices rather than how technology can serve as a tool to transform practice (Luckin, et al., 2012).

Transformative learning leads to change (Cranton, 1994), and change happens as previously held concepts are replaced with new ones (Schon, 1967). Examining teachers as learners, especially in relation to technology, can help to illuminate the process of learning as a method to transform teacher practices. It is important to develop a better understanding of what prevents teachers from adopting $21^{\text {st }}$ century instructional practices in order to better meet the needs of their $21^{\text {st }}$ century students. Although teachers have access to an ever-increasing number of technology devices and resources, teacher instructional use of additional laptops for one Midwestern school district has ranged from frequent to minimal, or has been non-existent, according to the chief of academics for the district (personal communication, November 7 , 2012). 
The superintendent of this school district has said no printed textbooks will be purchased when it is time to replace them (personal communication, October 2, 2012). Reallocating textbook funds to technology has cost the school district approximately one quarter million dollars (personal communication, December 10, 2012). Further, technology spending will continue to grow as school districts transition from an emphasis on print resources towards an increasing amount of digital resources. Yet concurrently, school leaders struggle to encourage teacher use of instructional technology tools with their $21^{\text {st }}$ century students. Instructional leaders from the Midwestern school district in this study would like to develop a better understanding of why some teachers embrace using the additional technology resources, while others do not. They would also like to know what they can do to encourage a transformation in teacher instructional practices towards their using technology tools more readily in their teaching.

\section{Research Problem and Purpose}

This study investigated the convergence of teachers' personal and situational influences affecting their use of instructional technology tools. Researchers have studied factors affecting teachers' use and resistance to use of instructional technology, but few studies have situated teachers at the center of their context (Luckin, 2010). Situating teachers as learners in the center of the professional context allowed for a holistic investigation of teacher pedagogy. While many studies illustrate the social nature of learning (Lantolf \& Appel, 1994; Luckin, 2008), few have examined teacher perspectives about their own learning and instructional technology use as a continuous change process. This study examined how factors combine to interact together to affect technology use within the teachers' ecological context, situating teachers at the center of the context. 
Previous research studies focused on fixed stages of change or levels of teacher technology use do not account for the continuous and cyclical nature of learning (Levin \& Wadmany, 2008). Numerous studies classify barriers to technology use as either intrinsic perceptions or extrinsic factors (Finger \& Houguet, 2009; Friedrich \& Hron, 2011; Howard, 2011), whereas it is better to combine these classifications and study the relationship among them (Levin \& Wadmany, 2008). A unity exists between situational and personal influences (Lantolf \& Appel, 1994; John-Steiner \& Mahn, 1996). Context is a key consideration, as is the role of teachers' life experiences and personal factors (Rogers, 2003). Factors such as gender, socioeconomic status, and number of years teaching interact with situational influences and factors, comprising facilitative and barrier filters affecting the dimensions central to the learner (Luckin, 2010).

The purpose of this study was to explore, understand, and describe high school teachers' views regarding the use of technology in their teaching practices specifically in science classrooms where laptops replaced new textbooks. The goal of this study was to seek understanding rather than to evaluate practice. It is important to develop a better understanding of teacher perspectives and pedagogy related to having access to additional instructional technology resources, especially in cases of limited use.

This study also sought to identify leadership actions and contextual factors needed to encourage teachers to use technology resources as instructional tools. Through examining teacher learning, we can help educational leaders better understand strategies to employ in order to have teachers transcend traditional teaching methods. Specifically, this study identifies and examines various filters as potential barriers to transforming practice and discusses what educational leaders can do to reduce those barriers. Also, this study provides recommendations for district and building leaders about what they can do to facilitate teachers' use of the 
additional technology resources, especially as the Midwestern school district under investigation increases its reliance on digital instructional resources. Drawing on research from the diffusion of innovation and adult learning literature, this study sought to explore teacher learning as a way to transform instructional practices related to technology.

\section{Research Questions}

The research questions that guided this study were:

1. How does the implementation of laptops in one high school's science classrooms affect teacher instructional practices?

2. What factors do teachers identify as facilitating or hindering their learning and instructional use of laptops in high school science classrooms?

\section{Conceptual Framework}

This study was guided by the ecology of resources framework (Luckin, 2010) situated within Vygotsky’s sociocultural learning theory (Wang, 2003) to frame the exploration of teacher use and non-use of the laptops in their teaching. Sociocultural learning theory encompasses the exploration of both the social and personal nature of learning as a cyclical process involving the complex relationship of culture and human interactions within specific contexts (John-Steiner \& Mahn, 1996; Lantolf \& Appel, 1994; Levin \& Wadmany, 2008; Luckin, 2008; Mahn \& John-Steiner, 2002). It provides the opportunity to examine intrinsic factors concurrently with extrinsic factors. Additionally, the ecology of resources framework allows for the consideration of context in relation to teacher technology use in the classroom, situating teachers as learners at the center of four relational dimensions affected by filters (Luckin et al., 2012). According to Luckin (2010), filters serve in oppositional roles as facilitators or barriers to the use of technology in teaching. Dimensions in which this study 
explored facilitative or barrier filters included: people, tools, knowledge and skills, and the environment. It is vital to understand each of the four dimensions and their interaction, as well as the teacher perspectives within the context as a whole.

Serving as the first dimension, people includes the school staff, those in the home setting, as well as those who comprise the school community. A community with a culture resistant to change is one example of a potential filter. Not understanding the powerful forces of human interactions on culture can cause us to become victim to them (Schein, 2004). Tools, the next dimension, can range from pencils and paper to computers, each supporting aspects of the learner’s cognitive processes (Lajoie, 2000; Shim \& Li, 2006). Filters within this resource can include time, money, and the availability of tools, according to Luckin (2010). As the third dimension, knowledge and skills are the content of what is to be learned. The curriculum is an example of a potential filter, because what schools teach must be limited in some way (Luckin, 2010). However, it must also be relevant. And as the fourth dimension, the environment includes settings, whether at home, school, or within the community. Possible filters include existing rules within those locales, as well as the nature of the environment itself (e.g., nurturing, rigorous, or culturally relevant) (Wang, 2003).

Exploring teacher use and resistance to use through the lens of Luckin's (2010) dimensions and the filters within them provided the ability to explore teacher perspectives in relation to their professional context. Luckin’s (2010) ecology of resources framework allowed me to focus my understanding on technology use in relation to the context of teaching, while investigating teacher perspectives holistically rather than in isolation. Integrating concepts of sociocultural learning theory within this framework enabled a more nuanced understanding of social interactions among teachers as adult learners, and the complex interactions among 
learners, dimensions, and filters (Friedrich \& Hron, 2011; Levin \& Wadmany, 2008; Li, 2010;

Luckin, 2010). According to Levin and Wadmany, we need more studies examining teacher use of instructional technology tools using concepts from sociocultural learning theory as a cyclical process of mediation and internalization within the teachers' context. Teachers' use of technology in their teaching progresses on a continuum, varying according to several factors. Concepts from sociocultural learning theory and the ecology of resources framework can combine to allow for the examination and exploration of the internal and external influences on teacher instructional technology use (Luckin, 2008; Luckin, 2010).

\section{Positionality}

My research perspective is situated within the constructivist paradigm. Thus, in my study I expected theories to emerge from my data in the form of patterns and relationships, as described by Grix (2004). When I interviewed the teachers, it was vital for me to clarify my role as a technology director and to address and identify possible bias as a result of that role. Due to my role and previous associations with the school district, it was even more essential for me to emphasize to participants my authentic interest in understanding and describing their viewpoints as I was guided by the theoretical and conceptual lens for this research. I was receptive to their perspectives as I listened intently. As the sole researcher of this study, I made every attempt to seek understanding from others in as unbiased a manner as possible, realizing I was developing understanding as I listened and carefully reflected upon the information I gathered from the participants (Weiss, 1994).

The choices I made to limit the scope of the information I attended to were guided by sociocultural learning theory (Lantolf \& Appel, 1994) and the ecology of resources framework (Luckin, 2010), as opposed to my role as a technology director. My assumptions and perspective 
working as a technology director helped me to better attune to the data I analyzed, as I remained diligently aware of my own assumptions and personal experiences. All researchers have biases, and we must strive to acknowledge them and limit them by employing research methods to minimize them (Stake, 2010). I also remained adaptive and open minded so I could view unexpected situations as learning opportunities as part of my study (Yin, 2014).

\section{Research Design \& Methodology}

This study provides an in-depth investigation of a problem related to practice. By utilizing a qualitative case study (Yin, 2014), this research provided the opportunity to more deeply understand teachers' range of use of classroom laptops in their teaching. By focusing on only one school setting, it allowed for a more nuanced, in-depth understanding of teachers’ perspectives related to the facilitative and barrier filters within their context. I utilized a single case study methodology to examine the common occurrence of teacher reluctance to embrace instructional technology practices (Yin, 2014). A case study methodology particularly suited this study because it sought to understand the process of educational technology use, rather than only describing various factors that may influence teachers' technology use. As called for by Guzey and Roehrig (2012), this study reveals further connections among factors affecting classroom practices and teacher learning, especially related to teacher motivation, beliefs, and knowledge. Using a holistic conceptual framework, this qualitative case study of science teachers from a large high school provides a better understanding of teacher perspectives related to using technology as a tool to transform their educational practices. Also, it helps to identify what school district leaders can do to increase facilitative filters and to decrease barriers to helping teachers make this change. 
Participants in this study were high school science teachers from a Midwestern school district that received enough laptops to achieve a two student to one device ratio within their classrooms. Through individual interviews, I explored teachers' views on receiving additional laptops instead of new print textbooks. I also conducted multiple classroom observations of those same teachers. It was important to talk with teachers to fully understand and describe their perspectives and pedagogy related to instruction and educational technology use. I supplemented the interview data with classroom observations, following strategies outlined by Wolfinger (2002) such as collecting field notes, and by attending to what is most noteworthy to my investigation.

\section{Significance of the Study}

This study adds a new perspective to the body of literature related to investigating teachers' use of instructional technology. By utilizing a holistic context within sociocultural learning (Lantolf \& Appel, 1994) and the ecology of resources framework (Luckin, 2008), and analyzing intrinsic and extrinsic factors in combination, it allows for a more integrated, deeper understanding of teacher perceptions related to their use of instructional technology resources in the classroom. As Levin and Wadmany (2008) posit, we need more studies examining instructional technology use as a learning process for teachers. Luckin et al. (2012) stress the need for teachers to be able to reach and engage students within their digital environment. By identifying facilitative filters within the dimensions comprising teachers' professional context, this study helps to inform educational leaders how to encourage teachers' use of instructional technology. Additionally, by identifying barrier filters within the teachers' context, it helps to address the problem of limited use, or nonuse of the additional laptops. Ultimately, this study reveals connections among the factors affecting classroom practices related to instructional 
technology use, especially related to teacher motivation, beliefs, and knowledge (Guzey \& Roehrig, 2012).

This qualitative case study addresses a problem from practice and helped one Midwestern school district's leaders, including the chief academic leader, curriculum directors, building leaders, and technology leaders better understand teacher pedagogy related to the additional technology in which they invested. It helped those leaders understand the differences in the range of use they had observed among the teachers who received access to the additional technology tools, while it helped them to understand what they could do as educational leaders to reduce potential barriers inhibiting teacher use of instructional technology. As Fullan (2012) stresses, “Technology is way too powerful for us not to have a plan.” (p. 72).

\section{Definition of Key Terms}

Several key terms were used throughout this study to describe teacher perspectives regarding instructional technology use within sociocultural learning and the ecology of resources framework. The following definitions will help readers more clearly understand their meaning. Definitions for more familiar terms indicate how they are being used within this specific study.

Context: The main component of Luckin's (2008) conceptual framework. It consists of four interrelated and filtered dimensions as it provides support systems and resources for learners. The dimensions include people, tools, knowledge and skills, and the environment.

Digital resources: Includes electronic information and tools, including but not limited to text books, web sites, reference databases, software, podcasts, images, videos, and devices such as desktops, laptops, and tablets, including iPads (McLeod \& Lehmann, 2012).

Instructional technology: The term to describe the use of digital tools and resources to improve teaching and learning in educational settings (ISTE, 2008). 
Mediation: In relation to sociocultural learning theory, mediation refers to the process of learners' interactions with specific external contexts as it continuously affects the internalization of learning (Levin \& Wadmany, 2008).

School culture: Includes a combination of environmental and social influences on the people within the educational environment, forming a collection of shared basic assumptions about how members of the group think, feel, act and perceive their environment (Schein, 2004).

\section{Conclusion}

Our schools need to experience an increased return on investment from the large sums being spent on instructional technology (Cuban, 2001; Johnson, 2011). As one Midwestern school district transitioned to an increased use of digital resources by providing additional laptops in high school science classrooms, it was vital for district leaders to understand teachers' use of instructional technology, especially because a wide range of use had been observed by district leaders (personal communication, November 7, 2012).

Many researchers have studied factors affecting teachers' use of instructional technology, but few studies have examined teacher perspectives within the center of their context (Luckin et al., 2012). Situating teachers as learners allowed for the opportunity to examine teacher viewpoints about technology use on a continuum, rather than according to fixed stages or levels of use as previous research has done (Levin \& Wadmany, 2008). Applying Luckin’s (2008) learner centric ecology of resources framework allowed this study to examine intrinsic and extrinsic factors in combination, while previous studies investigated these factors separately (Levin \& Wadmany, 2008).

By combining major concepts from sociocultural learning theory (John-Steiner \& Mahn, 1996; Lantolf \& Appel, 1994) and Luckin’s (2008) ecology of resources framework, I was able 
to explore teachers' cyclical nature of learning to use technology instructionally, while considering the teachers' context holistically, along with the relationships among the dimensions and filters affecting that context. This research contributes to the body of literature related to investigating instructional technology use from a holistic context (Luckin, 2010; Zhao \& Frank, 2003). It also contributes towards a deeper understanding of teacher perceptions related to the use of instructional technology in the classroom. Specifically, this qualitative case study sought to better understand science teachers' perspectives related to use of the additional laptops by using a combination of teacher interviews and multiple classroom observations. It addresses a problem from practice for school district leaders who were seeking to understand teacher practices regarding the additional technology in which the district had invested large sums of money. 


\section{Chapter 2: Literature Review}

The United States spent 9.7 billion dollars on technology for K-12 schools in 2013 (Center for Digital Education, 2013). However, according to Cuban (2001), “...computers in the classroom have been oversold by promoters and policymakers and underused by teachers and students.” (p.195). A recent international study on the impact of technology in education concluded a discrepancy exists between the digital environment students encounter in day to day life and their educational world, where teachers have been slow to embrace technology (Luckin et al., 2012). The researchers stress a critical need for the filters, or factors, facilitating and preventing teachers from using technological tools in the classroom to be explicitly examined, and subsequently addressed by all stakeholders. Among academics and practitioners there is an overwhelming call for schools to find ways to transform older teaching practices in order to harness the technology tools available to today’s students (Diem, 2010; Fullan, 2012; Keller \& Pearson, 2012; Kotrlik \& Redmann, 2009; McLeod \& Lehmann, 2012).

The resources available to today's students are changing, along with the way students interact with information. As Warlick posits, "We are preparing for a new generation of learners within a new information environment for a future that we cannot clearly describe” (McLeod \& Lehmann, 2012, p.1). An increasing number of school districts are transitioning from print to digital resources. Digital content is now readily available for use in today's classrooms (Ertmer, Ottenbreit-Leftwich, Sadik, Sendurur \& Sendurur, 2012). As school districts reallocate resources to electronic tools, schools need teachers willing and able to use these tools.

It is important to develop a better understanding of teacher perspectives and pedagogy related to the additional technology tools, especially in cases of limited use. This case study examined teacher views and practices related to the use of technology as a tool to enable better 
instruction. This examination from the individual teacher level helps us to describe the phenomena more accurately, and as Evans (1996) says, "Honor the lived realities of the educators” (p. 91).

This literature review discusses the relevant literature and research studies within the topics relevant to this study (Galvan, 2012) as well as the theoretical and conceptual frameworks which guided this case study. Beginning with a synopsis of technology in education over the past 20 years, I discuss the challenges inherent with introducing innovations and the change process itself, while examining change from a growth perspective and discussing adult learning as a process of growth. Finally, I explore sociocultural learning theory as it relates to teachers thinking about their own learning, and how that lens can inform this case study from the ecology of resources conceptual framework.

\section{Educational Technology during the Past 20 Years}

Over the past 20 years there have been significant changes to the technology tools available to today's students. Advancements and changes to both devices and educational applications are advancing at exponential rates. The digital revolution experienced in schools mirrors that of society. Much like the Industrial Revolution, our world is changing rapidly as it transitions into a post-industrial society where information, entertainment, and communication are driving new forms of technology (Best \& Kellner, 2001, p. 1). The new machine age is exponential (Brynjolfsson \& McAfee, 2011) and technology is evolving at an incredibly fast rate (Best \& Kellner, 2001; Fullan, 2012; McLeod \& Lehmann, 2012). Both machine-based software applications and web-based Internet applications are now available for use in education.

The rise of the Internet. During the 1990's, the move to increase Internet access to schools was facilitated by the E-Rate program (FCC, 2013). School districts and the federal 
government invested large sums of money in order to insure all students had Internet access. The main emphasis of educational technology during the 1990's centered on the number of schools having Internet access and the number of devices available for student use. While the focus has been on providing access to devices and Internet access, research shows there is not necessarily a positive correlation between increasing the number of devices and teacher use in instruction (Cuban, Kirkpatrick \& Peck, 2001). As a result, many question the impact of technology on student achievement.

The No Child Left Behind act of 2001 sought to improve student achievement through the use of technology in our K-12 schools (Diem, 2010; Kotrilk \& Redman, 2009). There was demand at both the governmental and societal levels to increase the number of computers in classrooms as far back as 2003, despite debate about the educational value of them (Zhao \& Frank, 2003). By 2004, almost every state either adopted or referenced the National Education Technology Standards (NETS) for teachers and students, including alignment with curriculum and instruction (Bielefeldt, 2012; Kotrilk \& Redman, 2009). By 2006, 98\% of public school classrooms reported having Internet access (Diem, 2010). These statistics reinforce how the device-centered focus of the 1990's continued through the first part of the $21^{\text {st }}$ century, maintaining the emphasis on ratios despite the establishment of technology standards.

Student to computer ratios reached an all-time low by 2010 (Gray, Thomas, \& Lewis, 2010). However, a 2011 large scale survey of integrating technology in literacy instruction found access to equipment as a major barrier (Hutchison \& Reinking). In the short time since that survey was performed, technology devices have shifted from being complex and expensive to being mobile and simple in usability (Garcia, 2012; Hedberg, 2010; McLeod \& Lehmann, 2012). More recently, there is an increasing number of one-to-one computer projects (Fleischer, 
2012). These efforts are eliminating traditional barriers of access to technology in education.

The current trend is to enable wireless (WiFi) capabilities in schools and even on school busses in order to provide ubiquitous Internet access (Best \& Kellner, 2001; Center for Digital Education, 2013).

Benefits and perils of the Internet. The U. S. Department of Education reported several benefits of technology use in the classroom in 2008 (Diem, 2010). The Internet is valuable as a source of most current scientific information (Boles, 2011). However, at the same time, there is an undisciplined nature to the Internet (Fullan, 2012). The very essence of the Internet leads to a situation where it can be viewed as a valuable resource, or a source of possible harm and threat, especially to children.

Today's youth have grown up with the Internet. According to Diem (2010),

Over the years, American youth have proven that they are extremely knowledgeable and skilled at using technology especially the Internet and synchronous and asynchronous communication. Technology has become a part of their daily lives both at home and at school. (p. 161)

They are growing up in the era of "just in time answers" thanks to the availability of the Internet (McLeod \& Lehmann, 2012). There is a need for critical literacy to judge authenticity of information (Hennessy, Ruthven, \& Brindley, 2005; McLeod \& Lehmann, 2012). Boundaries between authors and readers have blurred in terms of creation and consumption of information. Being able to assess the accuracy of information is critical in today's society. Technology is redefining literacy (Diem, 2010), furthering the need to teach students to be critical consumers of information and to address this need in their educational curriculum.

Move from device centric focus to curriculum centric focus. There has been an evolution within the past few years to move away from the device centered focus of the early 1990's towards a more curriculum centered focus on technology (Center for Digital Education, 
2013). There is also an increasing move towards digital content and curriculum (Fullan, 2012); however, Hutchison and Reinking (2011) found it was perceived as playing a supplemental role in literacy instruction rather than a central role. Technology continues to be viewed as an add-on to instruction in the majority of schools today.

There is a definite need to focus on technology to improve instruction rather than finding a way to use the technology (Center for Digital Education, 2013). There is also a need to move towards interdisciplinary instruction, especially in the science area with the STEM initiative for science, technology, engineering, and math (Keller \& Pearson, 2012). The focus must be about using modern tools to improve learning and increase thinking at a higher level (Diem, 2010; Ertmer \& Ottenbreit-Leftwich, 2010; Fullan, 2013; Lajoie, 2000; Luckin et al., 2012). Additionally, the new Common Core State Standards call for students to critically evaluate and use $21^{\text {st }}$ century text, and to produce information using technology (Avila \& Moore, 2012; Dalton, 2013; Drew, 2013). Ubiquitous access to computers and one-to-one computing environments are resulting in changing relationships between students, teachers, and administrators (Spires, Oliver, \& Corn, 2011). When technology is used as a learning tool, studies are showing an increase in student achievement (Lei \& Zhao, 2007) and the facilitation of understanding of science concepts and improved scientific reasoning skills (Dani \& Koenig, 2008).

Technology as a tool for learning. With the increased number of student devices, Internet access, and ever-increasing ubiquitous wireless access in schools, there is greater opportunity to engage students using technology tools. Technology tools not only support learning and engage students; they also enrich learning experiences by allowing opportunities for 
critical thinking, inquiry, and discovery (Diem, 2010; Fullan, 2012). Today more than ever before there is an increasing number of tools available for educational use. I discuss each of these tools below.

Web 2.0 tools. Web 2.0 tools are web pages allowing for more interaction and collaboration. They provide tools for the classroom, facilitating active involvement rather than passive consumption of information. These web pages include social media tools, enabling people to connect and form groups to meet and collaborate virtually with others from around the world (Betrus, 2012; McLeod \& Lehmann, 2012). They also include such resources as Twitter, a text-based communication tool, Blogs, which are web-based journals, and RSS readers which are Internet based feeds of information customized by and delivered to individuals (McLeod \& Lehmann, 2012).

Podcasts and webinars. Podcasts and webinars allow for video enabled meetings delivered via the Internet (Boles, 2011; McLeod \& Lehmann, 2012). They can connect learners with information either via audio or video transmission, enabling students to access information from teachers or experts when needed or desired. These technology tools help to facilitate anywhere, anytime learning.

Online courses. Online courses are also known as virtual schooling, and the term can include blended courses which combine face-to-face instruction with online instruction. Blended courses mean that 30 to $80 \%$ of instruction is delivered via the Internet (Christensen et al., 2008). Computer-based delivery of instruction is one of the fastest growing trends (Moeller \& Reitzes, 2011). Some researchers predict it will comprise half of all k-12 education by the year 2020 (McLeod \& Lehmann, 2012). However, online learning at this point is mainly about access, not instructional methods (Fullan, 2012). 
"Flipped" classrooms. The term "flipped" classrooms refers to technology-enabled structuring of instruction so teacher lecture or delivery of information occurs outside the classroom, often as assigned homework. It allows face-to-face class time to be dedicated towards allowing for more collaborative use of time, whether among students, or between teachers and students (Fullan, 2012). The "sit and get" delivery of instruction occurs outside the classroom via technology delivered methods. According to Fullan (2012), school leaders need to be placing an increased emphasis towards encouraging teachers to restructure their use of instructional time.

The Khan Academy. The Khan Academy consists of a collection of over 3,000 effective instructional math videos have been viewed over 130 million times (Fullan, 2012). They provide video-enabled instruction via an expert on mathematical topics where students can watch the content over and over again until they understand the concepts. Fullan describes the Khan Academy as an excellent example of instructional delivery via $21^{\text {st }}$ century technology.

Educational gaming. Educational gaming as tool for delivering instruction provides repetition, reward, and reason to produce engaging content for students to problem-solve and participate in experiential learning (McLeod \& Lehmann, 2012). Gaming applications are helpful for educators to keep in mind for certain objectives and for training on certain topics, and McLeod and Lehmann advocate for their use at all academic levels. Through the use of engaging instructional games, we can increase feedback for teachers and students while providing rich learning experiences involving higher level thinking skills (Fullan, 2012). There are increasing efforts to expand its use in PK-12 education (Fullan, 2012; McLeod \& Lehmann, 2012). 
Global impact of technology. While technology tools can enrich and extend learning experiences for students, there is a capitalistic and global nature to the increasing number of technology tools being consumed by our schools, as evidenced by the 56 billion dollars of annual expenditures (Johnson, 2012). While school districts invest funds to provide access to technology, there are large numbers of students who do not have access to the Internet or devices at home (Diem, 2010). Diem uses the term digital divide to describe those who have access to technology tools at home or in general, and those who do not. Machado-Casas and Ruiz (2012) found this digital divide exists at school, and also at home, creating a, "multigenerational rift between parents and their children.” (p. 4)

From the global technology perspective, there are countries and cultures less technologically developed (Machado-Casas \& Ruiz, 2012). According to their research, Machado-Cass and Ruiz found this digital divide is exacerbated for immigrant families in the United States lacking access to technology while their students have access to technology at school. As a result, the global digital divide becomes a familial divide, creating communication challenges within families. Understanding the impact of these digital divides is important for educational leaders. However, it is also a call for them to explore ways to bridge these digital divides (Machado-Casas \& Ruiz). According to the Maastricht Global Education Declaration (2002), we all must develop an awareness of the complexities related to technology and its social, ecological, political, and economic issues. As Machado-Casas and Ruiz (2012) posit, “The differences and complexities of technology knowledge also must be considered as factors influencing education” (p. 11). Schools must continue to address the digital divide and the global nature of technology as they expand use of these tools in schools. 
As a tool for learning, technology allows for personalized learning and differentiated instruction (Fullan, 2012; Moeller \& Reitzes, 2011). According to Christensen et al., "Integrated software, more easily than textbooks, can incorporate pathways for different types of learners, as methods for teaching in these different ways become understood.” (p. 132). This type of individualized instruction is what many educational leaders have been attempting for decades, and it is possible now more than ever using technology tools. However, simply having the tools and access to the Internet and digital content does not mean teachers will use them (Cuban et al., 2001; Guzey \& Roehrig, 2012; Fullan, 2012). According to Fullan (2012), technology has permeated every part of society except education.

Only 8\% of teachers fully integrate technology into the classroom (Moeller \& Reitzes, 2011). Consequently, a mere $43 \%$ of students feel prepared to use technology in their work life (Moeller \& Reitzes, 2011). Additionally, only 60\% of teachers report using technology in their classroom (Moeller \& Reitzes, 2011). The organizational support for the use of technology in schools is badly underdeveloped (Moeller \& Reitzes, 2011). Our schools are not adequately preparing students to address society's needs for highly skilled workers in new and continuously evolving fields (Kotrlik \& Redmann, 2009). It is actually more about knowledge than technology (Brynjolfsson \& McAfee, 2011). Technology alone is not enough (Brynjolfsson \& McAfee, 2011; Christensen et al., 2008). The way schools use computers places an emphasis on devices rather than instruction, leading to a failed implementation (Christensen et al., 2008).

Researchers have been studying the implementation of new technologies for years. Investigations into why teachers have been slow to embrace new innovations have been 
documented by Evans (1996). As all areas of society call for a transformation in educational practices, it is important for us to better understand the nature of change and its implications for the people in schools affected by it.

\section{Implementing Innovation and its Related Challenges}

Diffusion of innovations. Change cannot be achieved without understanding the people involved (Rogers, 1962). Schon says, "Belief in the stable state serves primarily to protect us from apprehension of the threats inherent in change.” (p. 11). It is natural to fear change (Fullan, 2012), therefore resistance to change is natural as well (Zhao \& Frank, 2003). While resistance to change is a natural response, people vary in how they react to it (Evans, 1996; Schon, 1971). The rate of change is important and we must respect it for each individual (Evans, 1996). How people respond to change is affected by personality, life experience, and career experience (Evans, 1996). Response to change is not affected by age or years of teaching, and may or may not correlate with gender (Mueller, Wood, Willoughby, Ross, \& Specht, 2008). However, Kotrlik and Redman (2009) found a positive correlation with gender. In their survey of 539 teachers, they found female teachers used instructional technology practices more than male teachers.

Resistance to change has been found to be especially prevalent in schools because it puts pressure on existing teacher practices (Cohen, 1987; Cuban, 1986; Zhao \& Frank, 2003). We need to acknowledge that both a need for continuity and change are interwoven in our schools (Cuban, 1986). Because change is often viewed as loss (Evans, 1996), we must acknowledge that perspective, and then help encourage the capacity for adaptation. The "dissenting mavericks” in a group can be helpful to avoid group think (Fullan, 2008; Janis, 2011). There can 
be value in exploring their skepticism while encouraging change. Three types of obstacles or barriers to change include inertia, skepticism, and indifference, therefore requiring a push towards it and support (Fullan, 2012).

When supporting and facilitating change, Fullan posits, “...change really isn’t as hard as we thought if we capture people's interest and give them enjoyable, worthwhile experiences.” (2012, p. 77). While this quote may seem to oversimplify the change process, Fullan's point is change must engage the individual in all ways. Implementing change is most effective when it is driven by a problem or a need, especially needs of individuals (Evans, 1996; Rogers, 1996; Schon, 1967). Change fails when it does not get at the beliefs or needs of those who are impacted by it (Evans, 1996). Technology may have the ability to enrich educational experiences for students (McLeod \& Lehmann, 2012), but if teachers do not believe in its benefits, they will not use the technology, and they especially will not use it as a tool for improvement.

Disruptive innovations. The displacement of one innovation or technology with another in order to fulfill a need or improve a process is considered a disruptive innovation (Christensen et al., 2008; Fullan, 2012). Decisions about whether to adopt a new technology in education depend on the individual. It is important to understand motives and how decisions are made by those involved with the innovation (Schon, 1967). A system of dynamic tension exists when a person is introduced to a disruptive innovation (McLeod \& Lehmann, 2012).

The role of internal dissonance. Internal dissonance or tension results in the person experiencing a state of instability and concern because the new information does not match with past experience and knowledge (Christensen et al., 2008; Rogers, 1962; Schon, 1971; Schon, 1983). As the individual attempts to reconcile this tension, the displacement of concepts is 
central to the formation of new concepts, leading to new theories and beliefs as a continuous process (Schon, 1967). However, this reconciliation process must not be more than the person can handle confronting (Schon, 1971). Schon stresses it is also important to maintain a sense of self-respect and self-identity while in the process of change and during this time of cognitive dissonance. Learning to use technology can be humiliating and stressful as well as difficult and painful (Schein, 1992). Efforts must be made to preserve a person's sense of competence. Additionally, the value of the innovation must outweigh the risk or perceived risk for acceptance to occur (Rogers, 1962). If we are encouraging each individual teacher to embrace technology as a way to improve instruction, each person must see the value as greater than the risk and we must respect each person's self-efficacy during the process.

The role of perceived usability. The perceived usability of the innovation and selfefficacy may increase acceptance (Holden \& Rada, 2011; Ertmer \& Ottenbreit-Leftwich, 2010). Howard's mixed-methods study (2011) found teachers weighed the time cost of using instructional technology tools against their perceived benefit of the tools. When integrating knowledge and pedagogy to produce change, the innovation should be: a) irresistibly engaging; b) elegantly efficient and easy to use; c) technologically ubiquitous; d) steeped in real life problem solving (Fullan, 2012). Mueller et al. (2008) found specific, task-relevant, real life classroom applications of technology were key factors to teacher use where the perceived usefulness was more important than organizational support. Hsu (2012) found a positive correlation between a teacher's ability to use technology and their use of it in the classroom, especially if they know how to use it effectively in their content areas.

Compatibility with beliefs also increases acceptance and use (Hutchison \& Reinking, 2011; Mueller et al., 2008; Rogers, 1962). In some cases we need to help people confront 
realities and beliefs they would prefer to ignore and to lessen their anxiety and fear. Evans calls this process, "unfreezing” (1996, p. 56). Evans says that during the process of change we must be open to the realities of others (1996). Schein describes the need to clarify beliefs and assumptions, especially implicit ones (1985). Shared beliefs and assumptions among individuals are the essence of group culture (Bolman \& Deal, 2008; Schein, 1985; Schein 1992).

Importance of shared vision. Shared vision, shared experiences and systems for diffusion of the innovation are critical when implementing change (Fullan, 2008; Park \& Ertmer, 2008; Rogers, 1962; Schein, 1992; Schon, 1971). The role of school culture on change cannot be underestimated (Ertmer \& Ottenbreit-Leftwich, 2010; Evans, 1996; Kitchenham, 2009; Schein, 1985). Clarity of vision building is necessary, in addition to clearly articulating the change (Caffarella, 2002; Evans, 1996; Kopcha, 2010). Li found shared vision and common goals critical to the success of a one-to-one tablet program because of their effect on teacher receptiveness and ownership of the change (2010). Teachers were united around a common effort to improve their instructional methods.

Explicitly stating expectations for the change is also vital. A lack of expectations or feedback was found to be a major barrier to technology enhanced instruction (Park \& Ertmer, 2008). Having a whole-system policy and strategy is a key to success (Fullan, 2012). In their detailed national profile of over 1400 literacy teachers, Hutchison and Reinking (2011) found instructional technology use was viewed as being supplemental, and more technologically oriented than curricular. When performing a case study about one school district's laptop program, Anthony (2012) found instructional technology efforts often exist in isolation of district 
or school-wide improvement efforts, leading to contradictory system linkages. The teachers viewed the technology like an extra effort rather than as being an integral tool for instructional improvement.

The role of opinion leaders. Opinion leaders in the social system play vital role as part of the communication network (Rogers, 1962). From a system perspective, they must not be underestimated. The role of formal and informal networks must also not be overlooked (Schon, 1971). $\mathrm{Li}$ (2010) found the use of informal networks instrumental in facilitating receptiveness to change among teachers. However, all networks must play an important role in addressing opposition and resistance to the innovation, including facilitating substantive conversations about merits of the innovation or change (Evans, 1996). There are always both positive and negative consequences when adopting an innovation (Rogers, 1962). They should be explicitly discussed and shared. Implementing change in schools is not the logical, rational process posited by Taylor (1911) because schools are dynamic organizations with multiple personalities and perspectives. Basic assumptions of an organization, “...operate at the most unconscious, implicit depths” (Rogers, 1996, p. 43) and guide behavior.

The role of motivation. The role of motivation on behavior and the decision to accept change cannot be underestimated (Christensen et al., 2008). Bullying or direct attacks to accept the innovation backfire when it comes to complex change (Christensen et al., 2008; Fullan, 2008). Therefore, individuals must be motivated for the change to occur. Intrinsic motivation plays a more valuable role than extrinsic motivation (Deci, 1975). Mueller et al. (2008) found no significant effect of extrinsic motivation of teachers fully integrating computers in their random sampling of 389 elementary and secondary level teachers. However, intrinsic motivation stimulates and compels the individual (Christensen et al., 2008; Deci, 1975; Deci \& Ryan 1985; 
Fullan, 2012). Guzey and Roehrig (2012) found that teacher intrinsic motivation towards using instructional technology helped them overcome barriers they encountered, even when many of the barriers were environmental. The teachers' own motivation encouraged them to overcome the barriers, leading to their regular use of educational technology tools in their teaching.

The role of the environment. The role of the environment must also not be overlooked when implementing change because it is vital to changing teacher behaviors (Clarke \& Hollingsworth, 2002). Teachers are known for autonomy and privacy (Johnson, 1990). Therefore, the level of trust among workers and in the leader is vital, as well as the stress level of the school, staff morale, staff cohesiveness, and their experience with previous change and innovation (Evans, 1996). Social support and the social context are vital for successful innovation to occur (Fullan, 2008; Zhao \& Frank, 2003).

Social support and networks among professionals are very influential. Clarke and Hollingsworth (2002) found the role of "growth networks" to be instrumental to successful teacher professional growth while Guzey and Roehrig (2012) found professional collaboration vitally important to helping teachers change their beliefs and instructional practice. Cranton (1994) found adults prefer collaborative learning. Their research showed team teaching to be an effective method for helping teachers to change. When implementing technology, Levin and Wadmany (2008) found peer coaching to be a valuable method. Other successful methods include team teaching (Cranton, 1994), mentoring (Kopcha, 2010), peer observations (Ertmer \& Ottenbreit-Leftwich, 2010), modeling (Belland, 2009; Schon, 1983), and video conferencing and video reflection groups (Belland, 2009; Tripp \& Rich, 2012). Each of these approaches further supports the importance of collaboration and the environment's social context when implementing innovation in educational settings. 
Implementing educational innovation. When profiling 40 leading innovators in education, the Center for Digital Education (2013) found all educators stressed, “...that technology does not transform learning itself. It is an aid and a tool to effective, creative instruction that only can come from dedicated instructors that strive for innovation” (p. 9). We often allow the focus on technology to overshadow the need for changing teacher instructional approaches and practices.

We need instructors to serve as change agents and students to serve in the role of knowledge workers (Cranton, 1994; Ertmer \& Ottenbreit-Leftwich, 2010; Fullan, 2012; Hattie, 2009). Teachers are the key to effective technology use in schools. We need for them to serve as guides for students when it comes to technology use. According to Fullan (2012), no matter how many wonderful resources are available from the Internet, we need teachers to help guide students, leading them towards learning how to learn.

A willingness to learn and build commitment for change is vital for implementing innovation in schools (Fullan, 2008; Evans, 1996). Fullan talks about "change knowledge” (Fullan, 2012) which involves having the knowledge, skill and pedagogy to successfully put something new into practice. Developing a learning organization striving for continuous improvement should be our goal (Anthony, 2012; Marquardt, 2011). The best way to help others confront change is through continuous learning and education (Evans, 1996). As we strive to encourage teachers to change instructional practices using technology as a tool, we must help them understand their own learning as a method for change.

In his book, Stratosphere, Fullan (2012) calls for a convergence of technology, pedagogy, and change knowledge in order to bring authentic change to schools. As schools strive for improvement, especially related to teacher pedagogy, they are most apt to achieve it through 
learning (Marquardt, 2011). Fullan (2012) describes a new paradigm for how we think of pedagogy. It is different than the traditional definition of teacher methods and practices related to the art and science of teaching. Rather, Fullan's (2012) new pedagogy involves learning how to learn. According to Fullan (2102), by learning how to learn, both teachers and students can be better equipped to handle rapid change.

\section{Adult Learning as a Vehicle for Change}

Learning and change. If change happens as previously held concepts are replaced with new thoughts and concepts, then for adults to change, there must be exposure to new ideas and learning for transformation to occur. Change can be viewed as a growth and learning opportunity for teachers (Clarke \& Hollingsworth, 2002). Transformative learning leads to change (Cranton, 1994) and learning organizations are those best able to respond to change (Marquardt, 2011; Schein, 1992). The goal of adult learning is to actively and collaboratively reconcile new ideas with existing ideas and past experience (Cranton, 1994; Mezirow, 1991; Schon, 1983).

Adult learning. For optimal success, adult learning must be voluntary and self-directing (Even, 1987; Hennessy, et al., 2005; Knowles, 1970; York-Barr, Sommers, Ghere, Montie, 2006). The role of choice and self-direction cannot be underestimated (Beder, 1985; Cervero \& Wilson, 1994; York-Barr et al., 2006). In terms of participation, acquisition, and outcome, adult learning is voluntary. Researchers have found if learning is forced, non-learning and resentment result (Knowles, 1970; Wolcott, 1977). Just like with implementing change, you cannot force adults to accept a learning objective or change agenda but rather must engage each individual.

Motivation for learning. For many individuals, motivation to learn comes from the desire to successfully perform their professional roles and not necessarily because anything is broken 
(Beder, 1985; York-Barr et al., 2006). Additionally, Knowles found adult motivation for learning becomes more problem-centered with maturity (Knowles, 1970). Like with change, it is most successful when driven by a need that is compelling for the individual, whether the need be driven by a problem or desire to succeed professionally. In their three year study, Guzey and Roehrig (2012) found intrinsic motivation for each individual teacher was the most important factor for changing their professional practices to include technology.

It is important to recognize, "the idiosyncratic and individual nature of teacher professional growth” (Clarke \& Hollingsworth, 2002, p. 947). Just like when implementing change, we must acknowledge fear as a part of adult learning (York-Barr et al., 2006). Some people are more likely to be open to learn while others need different levels of support from multiple people such as co-workers, experts, friends and family (Caffarella, 2002). Additionally, adult learners in the teaching profession must make conscious choices about dedicating their time to learn because time is an especially valuable resource in schools (Cuban, 1986; York-Barr et al., 2006). Individual teachers make different decisions regarding the amount of time they are willing to dedicate to their learning. Subsequently, they will make the same decisions regarding their learning about technology.

Reflection and learning. Ideally, teachers are active, reflective participants in their learning (Caffarella, 2002; Clarke \& Hollingsworth, 2002; Davenport, 1984; Fullan, 2008; Jones \& Moreland, 2004; York-Barr et al., 2006). The role of critical reflection and action are vital components for learning and change to occur (Fullan, 2008; Schon, 1983). Guzey and Roehrig (2012) found critical reflection involving active and continuous evaluation of daily instructional practices and student learning increased science teachers' use of instructional technology. Critical reflection brings another dimension to simple reflection, requiring the learner to evaluate 
the new information within some context, ideally evaluating it in relation to current knowledge within their professional setting. Adult learning literature verifies the importance of context and learning within one’s own setting where the content is more relevant (Caffarella, 2002; Fullan, 2008; Killian \& Killian, 1983; York-Barr et al., 2006). Using the lens of teacher as change agent, Ertmer \& Ottenbreit-Leftwich (2010) reviewed the literature detailing the factors necessary for effective technology use among teachers. They found teacher knowledge and school culture combine to affect the teacher's situational context for learning.

Within schools, the teachers' context is their classroom. However, the teachers' isolation within their separate classrooms presents a challenge for reflective, collaborative learning (Schon, 1983). The importance of purposeful peer interactions has been documented within much of the adult learning literature (Ertmer \& Ottenbreit-Leftwich, 2010; Fullan, 2008). Similar to findings in the diffusion of innovation literature, trust and supportive social conditions foster reflective practice among teachers (Hoy, Gage, \& Tarter, 2006; Podeschi \& Pearson, 1986; York-Barr et al., 2006; Zhao \& Frank, 2003). Appropriate social conditions must exist for risk taking, which is an important component of applying learning and adapting it to current experience (Ertmer \& Ottenbreit-Leftwich, 2010).

The role of experience in learning. The role of experience and teachers' divergent experiences are critical (Caffarella, 2002). Prior adult experience is a valuable resource for learning (Knowles, 1970). Likewise, being able to directly apply their learning is vital for adults. Beder (1985) documented the important role of utilizing both formal and informal learning activities with adults, and the need for immediate application. However, professional development alone will not increase teacher use of instructional technology (Mueller et al., 2008). Because adult learning involves reconciling new ideas with existing practices, teachers 
must have a willingness to try new activities and methods as part of their learning. Within a safe, learning-oriented environment, teachers can be encouraged to apply their learning about technology, ideally resulting in changes to instructional practices and pedagogy (Fullan, 2012)

Pedagogy and change. Pedagogy can vary by gender, educational setting, and content discipline (Davenport, 1984), as well as by culture, socioeconomic status and school organizational structures (Harris \& Hofer, 2011). Teachers’ pedagogical stance may be the best determination of their propensity towards using instructional technology (Diem, 2010; Ertmer, 2005; Jones \& Moreland, 2004). Other researchers have found an intersection between technology, pedagogy, and content knowledge (Guzey \& Roehrig, 2012; Harris, Mishra \& Koehler, 2009; Jones \& Moreland, 2004). Fullan (2012) stresses it is important to keep in mind that not all technology is good for pedagogy, and great pedagogy can exist without technology. The key is to examine and improve pedagogy through learning in order to apply methods where technology can enhance instruction.

Changing pedagogy and using technology as a tool can enable $21^{\text {st }}$ century instructional methods involving active student participation and collaboration (Fullan, 2012). If personal development and learning occurs from the interaction of internal subjective forces and external circumstances as posited by Jung (1971), then examining teachers’ views about their learning, pedagogy, and use of instructional technology within their environments can provide insights about how educational leaders can facilitate this change. Zhao and Young (2003) identified the need to focus on teacher level factors affecting their technology use. Because learning and critical reflection are vital for change to occur, and reflection can change pedagogy, it is time to be asking how we can encourage a change in teacher practice towards a more $21^{\text {st }}$ century orientation, rather than how we can increase teacher use of instructional technology. 
Fullan (2012) has advocated for a new pedagogy where teachers focus on learning how to learn, specifically their own learning, as a way to improve instruction. As Schon posits, "We must, in other words, become adept at learning” (1971, p. 30). Sociocultural learning theory provides an effective lens for examining teachers as learners striving to improve their instructional practice and knowledge. It can allow us to develop a deeper understanding of teacher beliefs about their own learning. Additionally, it can help investigate how their learning is affected by personal and situational influences.

\section{Exploring Instructional Technology Use through Sociocultural Learning Theory}

Sociocultural research focuses primarily on the co-construction of knowledge and how learning is internalized, appropriated, and transmitted in formal and informal learning settings (John-Steiner \& Mahn, 1996). Using Vygotsky’s sociocultural learning theory as a lens to explore teachers as adult learners enabled me to consider the importance of context to learning (John-Steiner \& Mahn, 1996; Wang, 2003). It allowed for the investigation of learning embedded within a network of relationships with individuals and the environment (Lin, 2011). While many studies have investigated barriers to teacher use of technology in their instruction, few have been done so using sociocultural learning theory and the ecology of resources framework. Because there is not a simple explanation for why teachers choose to use or not use technology as a tool for instruction, it is important to facilitate a deeper understanding of teacher learning and perspectives regarding instructional technology use (Ertmer, 2005; Hutchison \& Reinking, 2011; Levin \& Wadmany, 2008; Lin, 2011; Sheffield, 2011; Zhao \& Frank, 2003).

Sociocultural learning theory includes the exploration of both the social and cyclical nature of learning, along with the complex relationship of culture and human interactions within specific contexts (Lantolf \& Appel, 1994; John-Steiner \& Mahn, 1996; Levin \& Wadmany, 
2008; Luckin, 2008). Within Vygotsky’s ontological perspective, humans are mediated beings with their environment (Lantolf, 2006; Wang, 2003). John-Steiner and Mahn have applied this theory to learning and development, concluding learning is co-constructed and contextual. Luckin (2010) has also applied these concepts to technology and its use in the classroom, emphasizing the importance of context for developing rich learning activities. The main concepts from sociocultural learning theory include the social nature of learning, the Zone of Proximal Development, and the mediation and internalization of new concepts and learning (John-Steiner \& Mahn, 1996; Lantolf \& Appel, 1994; Luckin, 2008).

The social nature of learning. Social interactions are key components to learning, forming a dynamic relationship between social and individual processes (John-Steiner \& Mahn, 1996; Luckin, 2008). Learning is inherently social as cultural factors and human interactions combine to form the contextual environment for the individual (Lantolf \& Appel, 1994; Levin \& Wadmany, 2008; Luckin, 2008). Both culture and human interactions are important to learning as key components of one’s context (Ertmer \& Ottenbreit-Leftwich, 2010; John-Steiner \& Mahn, 1996; Schein, 1992). The role of context is a key factor in learning for both individuals and groups (John-Steiner \& Mahn, 1996; Luckin, 2008). Schools are social organizations; therefore, a teacher's environment within the contextual school setting can have a significant effect on learning (Zhao \& Frank, 2003). Cultural factors within a setting involve interactions between a person with others, a person with the physical world, and a person with one's own internal mental world (Lantolf, 2006). Vygotsky saw imitation as a method for transforming observed behaviors into learned behaviors (Lantolf, 2006). Levin and Wadmany (2008) use the term enculturation to describe the process of teachers learning through a combination of experience, observation, and direct instruction with others. 
The Zone of Proximal Development. The Zone of Proximal Development (ZPD) is what a learner can do alone, and what a learner can do with assistance from others as well as from tools (Brown, 1992; Lantolf \& Beckett, 2009; Luckin, 2008). Vygotsky viewed the ZPD as the role of more knowledgeable partners assisting with the development of the learner until the point when the assistance is no longer needed. The role of more knowledgeable partners in the development of individuals is important to learning within the ZPD until the learning is internalized (John-Steiner \& Mahn, 1996). In their three year study of views and factors affecting teacher use of instructional technology, Levin and Wadmany (2008) found external supports vital for teachers during the initial stages of their learning.

External supports that can provide scaffolding play a vital role in the learning process (Luckin, 2008). Li (2010) found informal networks very important in supporting teachers with instructional technology use. Zhao and Frank (2003) found the informal help and associations teachers form have as much influence on their technology use as other more frequently examined factors. Most notably, they found help from close colleagues was more significant than help from trainers or other people. What matters the most for teachers is their peers in the local environment (Ertmer \& Ottenbreit-Leftwich, 2010). Thought and conversation also play a key role in the learning process (Lantolf \& Appel, 1994; Levin \& Wadmany, 2008). Over the course of their study, Levin and Wadmany (2008) observed teachers move from dialogue with peers about technology use to personal reflection on their own instructional practices. Consistent with this finding and adult learning literature, collaboration with others has been found to be another facilitating factor to learning (Guzey \& Roehrig, 2012; Luckin, 2010). When scaffolding learning, the right amount of support from others at the right time facilitates learning and helps 
prevent the learner from being overwhelmed (Luckin, 2010). Consistent with diffusion of innovation literature, the rate of change or learning must be appropriate for each individual (Evans, 1996).

Mediation and internalization. For each individual, the nature of learning through the mediation and internalization process involves a cyclical and continuous interaction between themself and their external world (Lantolf \& Appel, 1994; Levin \& Wadmany, 2008; Luckin, 2010). Wertsch (1994) explains mediation from a Vygotskian perspective as, “...how human mental functioning is tied to cultural, institutional, and historical settings since these settings shape and provide the cultural tools that are mastered by individuals” (p. 204). Mediation with the external context continuously affects the internalization of learning (Levin \& Wadmany, 2008). Levin and Wadmany found teachers’ learning and changes in perspective about instructional technology progressed on a continuum rather than by fixed levels or stages. This finding aligns with the mediation and internalization of learning where individuals continuously revise and update the processing of information from their environment, relating it to their personal, internal world.

Situational influences. Situational influences from one’s environment directly influence the mediation process. They include social capital (Li, 2010; Zhao \& Frank, 2003), social pressure (Li, 2010; Zhao \& Frank, 2003), and the degree of trust within the organizational environment ( $\mathrm{Li}, 2010)$. These influences affect the working environment for teachers. Li (2010) posits they exert great influence on collaboration and learning within a school setting.

Social capital. Zhao and Frank (2003) define social capital as the amount of help teachers are willing to provide colleagues within their organizational setting. Li (2010) found social capital to be a pivotal factor in the success of a one-to-one computer initiative. Li defines social 
capital as the informal social structure of the school. While Li's definition of social capital is more general than Zhao and Frank's, the common theme of the informal social network's effect on teacher learning is the salient point, corresponding directly to both the diffusion of innovation and adult learning literature.

Social pressure. Social pressure can involve both exerting pressure on others or conforming to pressure from others (Zhao \& Frank, 2003). Li (2010) examined the informal social processes’ effect on technology use within the school setting. Social pressure proved to be a decisive factor facilitating the use of the technology tools by the teachers in Li's case study of a one-to-one laptop initiative. While the diffusion of innovation literature has found pressure should not come from leaders, this finding shows it may be more productive coming from colleagues.

Degree of trust. Li (2010) also found social trust important to the success of teachers’ willingness to experiment with innovational methods related to the new laptops. The perception of trust within the school setting is also important to the actual overall feeling of trust. According to Hoy et al., (2006), “A culture of trust should provide a setting in which people are not afraid of breaking new ground, taking risks, and making errors” (p. 237). Trust must involve listening to each other and making it safe to share concerns and fears. It involves the confidence one’s colleagues and leaders will show respect and consistency between words and actions (Li, 2010). Consistent with adult learning literature, appropriate social conditions must exist for individuals to feel safe taking risks (Ertmer \& Ottenbreit-Leftwich, 2010).

Personal influences. Current and historical personal influences for individuals also affect the internalization of learning process. The role of life experience is critical (Mahn \& JohnSteiner, 2002). Gender, socioeconomic status, and culture affect a teacher's knowledge and 
practice (Harris \& Hofer, 2011). But there is no correlation to age or number of years teaching (Mueller et al., 2008; Shieh, 2012). For each individual, the interaction between affective feelings and cognitive perceptions play a vital role in the willingness to embrace learning as a vehicle for change (Lantolf, 2006; Mahn \& John-Steiner, 2002). The same holds true for teachers' willingness towards learning to use technology as a tool to improve instruction. Their affective feelings and perspectives play a large part in their learning (Diem, 2010).

Affective factors. Affective factors such as confidence (Mahn \& John-Steiner, 2002), anxiety, and fear play a role in one's willingness to learn, thereby affecting one's openness to change (Diem, 2010; Howard, 2011). For instance, Kotrilk and Redman (2009) found as technology anxiety decreased, teacher technology use increased. A lack of confidence is a normal part of learning, but successful learning can be facilitated by the situational factors of trust and social capital (Levin \& Wadmany, 2008). It is important to remain cognizant of these considerations. Levin and Wadmany performed an in-depth, longitudinal case study to explore teacher views on factors affecting their instructional technology use. Their study uncovered the importance of maintaining sensitivity to teacher views, thoughts, and situational influences in order to improve their learning to use technology instructionally.

Cognitive factors. The cognitive factors of conscious and unconscious thought also influence the internalization process of learning (Mahn \& John-Steiner, 2002). Passion for technology (Ertmer et al., 2012), knowledge and skill (Ertmer et al., 2012), problem solving ability or mentality (Ertmer et al., 2012; Howard, 2011), and beliefs and values (Ertmer, 2005) exert influence on the internalization of learning and change. Numerous studies have examined the significant role teacher beliefs and values play in teacher pedagogy and willingness to learn (Friedrich \& Hron, 2011; Howard, 2011; Levin \& Wadmany, 2006; Levin \& Wadmany, 2008; 
Mueller et al., 2008; Petko, 2012). Hutchison \& Reinking’s (2011) broad, large scale survey of technology integration showed teacher beliefs may not pose obstacles to use but that conclusion warrants further investigation. However, a belief of instructional technology's importance correlates positively with its use (Guzey \& Roehrig, 2012; Hutchison \& Reinking, 2011; Mueller et al., 2008).

A belief in the innovations' importance corresponds to findings from the diffusion of innovation literature. Many themes from that body of research such as motivation, a sense of competence or professional efficacy, and perceived usability correspond to cognitive factors affecting learning. Motivation towards using technology is facilitated by showing evidence of its benefit to students (Ertmer et al., 2012). Knowledge and skill include professional efficacy and a sense of professional competency in using technology tools (Deci \& Ryan, 1985; Finger \& Houguet, 2007; Howard, 2011; Hutchison \& Reinking, 2011; Petko, 2012). The ability to deal with the unexpected is also an important factor (Levin \& Wadmany, 2008) because as Rogers (1962) found, there will be both positive and negative consequences when working with innovations.

Risk perceptions. Howard's study (2011) of teachers' risk perceptions about innovative technologies found teachers had legitimate concerns grounded in their beliefs. These teachers placed a lower value on instructional technology as a result. Likewise, professional efficacy corresponded positively with the use of instructional technology (Howard, 2011; Mueller et al., 2008; Petko, 2012). Overall, a compatibility with cognitive factors increases a teacher's willingness to learn new skills. Specifically, nuances within teacher beliefs and attitudes affect their willingness to use technology in the classroom (Donnelly, D., McGarr, O., \& O’Reilly, J., 2011; Ertmer et al., 2012; Finger \& Houguet, 2009; Howard, 2011; Hutchison \& Reinking, 2011; 
Mueller et al., 2008). In their study of science teachers' use of instructional technology, Donnelly et al. (2011) found teachers' sense of empowerment and ownership increased their technology use while a sense of helplessness and fatalism did not. As Fullan (2007) says, "what teachers do and think...” affects the use of educational innovation (p. 129). The TechnologyEnabled Active Learning (TEAL) study designed to assess the effect of this innovative method for delivering technology-enhanced instruction, found teachers who think knowledge can be imparted to students lean towards emphasizing traditional lectures (Shieh, 2012). After spending two years performing classroom observations of instructional technology use by teachers, Bielefeldt (2012) concluded the amount of time they use instructional technology is directly related to their inclination towards whole group or small group instruction, with technology being more critical to learning when used during small group instruction. Levin and Wadmany (2008) found it is possible to change teacher beliefs and educational practice through learning. They found, “...the use of technology in teaching and the changes that occur in their beliefs and practices are linked to the different patterns of teachers' learning” (p. 253).

When examining teachers as learners, there is a unity between situational and personal influences (Lantolf \& Appel, 1994; John-Steiner \& Mahn, 1996). Vygotsky emphasized the indivisible unity of personal and situational characteristics (Vygotsky, 1978). These influences exist in combination for learners. Context is a key consideration (Luckin, 2008), including how learning is internalized to the point of needing limited mediation for each person. $\mathrm{Li}$ (2010) advocates for the need to deepen knowledge about the complexity of technology diffusion, calling for studies examining the interplay between psychological, social and organizational factors. We must further examine the educational context and the impact of inter-related 
resource elements, as opposed to separate influences, in order to further our understanding about teachers’ use of technology as an instructional tool (Guzey \& Roehrig, 2012; Lin, 2011; Luckin, 2010; Luckin et al., 2012; Zhao \& Frank, 2003).

The Learner Centric Ecology of Resources Framework. The learner centric ecology of resources framework (Luckin, 2008) grounded within sociocultural learning theory (John-Steiner \& Mahn, 1996; Wang, 2003) allows for a more nuanced exploration of teacher instructional use and non-use of the laptops. I used this conceptual framework to investigate teacher perspectives as I situated them as learners within the framework. Through this lens I explored four main concepts: 1) teachers situated as the learners in the center of the context, 2) context as consisting of four relational dimensions of resources, 3) filters affecting the teacher and the four dimensions, and 4) complex interactions among dimensions and learners (Luckin, 2008; Luckin, 2010, Luckin et al., 2012).

Teachers situated as learners in the center of the context. Teachers are situated as learners at the center of the educational context. Researchers have studied factors affecting teachers' use of instructional technology as well as resistance to use, but few studies have situated teachers as the learners at the center of a holistic context (Luckin et al., 2012). It is important to take a holistic view of adult education (Sample \& Kaufman, 1986). Friedman and Hicks (2006) stress the need for researchers to analyze the needs of teachers in order to understand their Internet use in the classroom while other researchers stress the need to understand the underlying factors affecting teacher technology use (Wu, Chang, \& Guo, 2008). Hew and Brush (2007) call for the need to study the larger context of both the school and district levels affecting teachers. Applying activity theory, Anthony (2012) views teacher technology use as a network of inter-related systems affecting its use. He calls for studying the linkage of 
district systems affecting it (Anthony, 2012). The ecology of resources framework considers context in relation to teacher technology use in the classroom, situating teachers as learners at the center of four linked, relational dimensions affected by filters.

Previous studies focused on levels of technology use do not fully allow for the cyclical nature of learning in relation to using technology instructionally (Levin \& Wadmany, 2008). Teacher learning is not a linear process, they posit. Donnelly et al. (2011) call for studying mixed stages to more accurately account for the mixed teacher stances they found in their study of chemistry teachers. The ecology of resources framework allows for investigating the cyclical nature through which personal learning progresses, and how it relates to the dimensions affecting the teachers' context and their evolving use of instructional technology (Lantolf \& Appel, 1994; Levin \& Wadmany, 2008; Luckin, 2010).

Many previous studies have been technocentric but there is a need to focus on the dynamic relationships and pedagogy affecting teacher technology use within their curriculum (Harris et al., 2009). We need to conceptualize instructional technology use as a learning process for teachers (Lawless \& Pellegrino, 2007; Levin \& Wadmany, 2008) and not simply focus on environmental factors (Mueller et al., 2008). Teacher knowledge is a significant factor (Ertmer \& Ottenbreit-Leftwich, 2010). When we view teachers as students within Luckin’s (2008) ecology of resources framework, we can examine how their personal factors interact with the situational factors within the dimensions impacting their learning process (Lantolf \& Appel, 1994; Luckin, 2008). As Luckin (2008) posits, the learner's context is defined as the specific circumstances involving the student and social interactions, with each being oriented both historically and culturally. 
Context as consisting of four relational dimensions of resources. Context is the main component of Luckin's (2010) conceptual framework. Consisting of four dimensions, it provides support systems for student learning while these resources are simultaneously filtered. The filters between the learner and the resources can be helpful filters, or potential barriers. The four interrelated dimensions within the context include people, tools, knowledge and skills, and the environment (Luckin, 2008).

People. The first resource is people and includes the school staff, those in the home setting, and those who contribute to the culture of the school. Human interactions exert the most influence on the learning context (Diem, 2010; Li, 2010; Zhao \& Frank, 2003). Within this dimension, stakeholder involvement is an important key to success (Levin \& Wadmany, 2008; $\mathrm{Li}, 2010)$. Additionally, there is a need for a human infrastructure in support of instructional technology use (Lin, 2011).

Tools. Tools, the second resource, can range from pencils and paper to computers, each supporting aspects of the learner’s cognitive processes (Lajoie, 2000; Shim \& Li, 2006). They include physical artifacts such as hardware and software, as well as both printed and digital information (Finger \& Houguet, 2009; Levin \& Wadmany, 2008). As evidenced by many oneto-one computer initiatives in our schools, technology tools are becoming increasingly available and this increased availability provides for easier access (Johnson, 2011; Luckin et al., 2012).

Knowledge and skills. Knowledge and skills are the content of what is to be learned, with relevancy being an important consideration (Luckin, 2010). High quality professional development is important for teacher growth (Ertmer, 2005). However, Anthony (2012) found some instances where professional development actually inhibited the use of instructional technology. Anthony calls for evaluating learning goals and motives, while advocating for 
individual professional learning goals. Levin \& Wadmany (2008) stress the importance of acknowledging a lack of confidence and uncertainty as normal characteristics of learning. It is important to empower teachers to experiment with knowledge application (Levin \& Wadmany, 2008; Li, 2010; Petko, 2012) and to develop organizational knowledge (Underwood, Smith, Luckin, \& Fitzpatrick, 2008).

Environment. As the fourth resource, the environment includes settings, whether at home, school, or within the community. The teacher's educational environment includes norms, expectations, and school culture (Levin \& Wadmany, 2008). A collegial environment is a key factor in helping teachers mediate and internalize learning related to technology (Finger \& Houguet, 2009; Friedrich \& Hron, 2011; Levin \& Wadmany, 2008). This dimension has a significant influence on teachers' instructional technology use (Guzey \& Roehrig, 2012) as it serves a key role in the mediation process of learning (Luckin, 2008; Luckin, 2010).

Filters affecting the teacher and the four dimensions. A dynamic process exists within the context where multiple filters impact each of the four the dimensions, thereby affecting the mediation and internalization of learning (Luckin, 2008; Luckin, 2010). The filters between the learner and the resources can be helpful filters, or potential barriers. It is important to identify and examine the filters within each dimension while recognizing the same filter can serve in a dual capacity as either a facilitative filter, or as an obstacle, depending on the situation. Luckin (2010) posits each of these filters has a significant impact on the learner and their use of technology tools.

Filters affecting the people dimension. Facilitative filters affecting the people dimension can include (a) mentors, peer coaches and experts (Levin \& Wadmany, 2008); (b) influential others who provide helpful strategies ( $\mathrm{Li}, 2011)$; (c) peers who form a positive informal social 
context (Zhao \& Frank, 2008); and (d) supportive administrators (Guzey \& Roehrig, 2012; Hutchison \& Reinking, 2011). Barrier filters affecting the people dimension can include (a) school leaders, stakeholders, or peers who demonstrate a lack of support or empowerment (Li, 2010); (b) a prevalence of fear (Diem, 2010; Evans, 1996; Fullan, 2012); and (c) a lack of motivation to use technology instructionally (Hutchison \& Reinking, 2011). The people affecting a school environment have the ability to help facilitate teacher use of instructional technology, or they can pose as barriers to it.

Filters affecting the tools dimension. Facilitative filters affecting the tools dimension include (a) the increasing abundance of technology devices (Li, 2010, Luckin et al., 2012; Petko, 2012); and (b) a good fit between the available technology tool and the content area (Harris \& Hofer, 2011). Barrier filters can include (a) the high cost of technology devices (Johnson, 2012); (b) the complexity of some devices or software programs (Fullan, 2012; Howard, 2011); and (c) the amount of time to learn to use devices or software programs (Howard, 2011). The tools available within a school can facilitate teacher use of instructional technology when they are readily available and easy to use, or they can serve as barriers.

Filters affecting the knowledge and skills dimension. Facilitative filters impacting the knowledge \& skills dimension include (a) curriculum embedded, on-going technology professional development programs rather than a focus on simply how to use the tools (Guzey \& Roehrig, 2012; Hutchison \& Reinking, 2011; Li, 2010; Levin \& Wadmany, 2008); (b) informal and formal access to expertise (Li, 2011); and (c) pacing delivery of instruction appropriately for each person (Levin \& Wadmany, 2008). Filters serving as potential obstacles within the knowledge and skills dimension include (a) a lack of opportunities to apply new information and skills (Finger \& Houguet, 2009; Petko, 2012); (b) a curriculum where there is too much to cover 
(Cuban, 1986; Diem, 2010; Hennessy et al., 2005); (c) unfocused professional development offerings or sessions with a cookie-cutter approach (Anthony, 2012; Hutchison \& Reinking, 2011); and (d) the inappropriate pacing of the delivery of instruction for each person (Levin \& Wadmany, 2008). These examples show how the content of what is to be learned and the way learning is approached can either facilitate teacher use of instructional technology, or present barriers to its use.

Filters affecting the environment dimension. Within the environment possible filters can include existing rules within locales, as well as the nature of the environment itself (e.g., nurturing, rigorous, or culturally relevant) (Wang, 2003). Facilitative filters include (a) a high amount of social capital (Anthony, 2012; Li, 2010; Zhao \& Frank, 2003), (b) numerous available scaffolds (Li, 2010; Luckin, 2010)), (c) the positive influence of colleagues through collaboration (Diem, 2010; Guzey \& Roehrig, 2012), (d) longer class periods such as block schedules (Hew \& Brush, 2007), and (e) school policies providing for access to resources (Finger \& Houguet, 2009; Petko, 2012). These facilitative filters provide the supports and structures needed to encourage instructional technology use within a school. At the same time, barrier filters such as (a) school policies restricting access to resources (Friedrich \& Hron, 2011); (b) a lack of social trust (Hoy et al., 2006; Li, 2011); (c) a legacy of negative school culture (Friedrich \& Hron, 2011); (d) the negative influence of colleagues (Diem, 2010); (e) an inflexible school day timetable (Hew \& Brush, 2007); (f) a lack of school planning related to technology use (Hew \& Brush, 2007); and (g) top-down policies that disempower teachers (Hennessy et al., 2005) present obstacles to the use of instructional technology tools. Most noteworthy about the environment dimension is the large number of filter-related examples found within the body of instructional technology literature compared to the other three dimensions. The existence of 
multiple barrier filters within any one dimension can have a negative impact on learners due to the interaction among the four dimensions (Luckin, 2010). For this reason, it is important to examine how the inter-relationship among filters affects the teachers' context.

Studying filters affecting technology use. Numerous studies have classified barriers to technology use according to either teachers' intrinsic (Wu et al., 2008) or extrinsic factors (Finger \& Houguet, 2009; Friedrich \& Hron, 2011; Howard, 2011), or they categorize barriers to change according to first-order and second-order barriers (Ertmer, 1999). Studies have also focused on teacher attributes without considering the teacher's context such as school culture. Hew and Brush (2007) provided a detailed analysis of the integration barriers, identifying 123 of them and categorizing them into six main categories: resources, knowledge and skills, institution, attitudes and beliefs, assessment and subject culture. Four of these areas, including teacher attitudes and beliefs, teacher knowledge and skills, the institution, and resources, directly affect teacher use; culture and assessment indirectly affect their use. While they offer strategies for overcoming barriers, they stress the need for additional studies to examine the dynamic relationship between barriers and strategies to overcome challenges.

Many studies illustrate success with improving teachers' use of instructional technology. Proven strategies for overcoming barriers can include conducting professional development (Hew \& Brush, 2007), reconsidering how we assess students (Hew \& Brush, 2007), and changing attitudes and beliefs through learning (Levin \& Wadmany, 2008). Additionally, time commitment to teaching and openness to change, combined with technology training were identified as predictors of teacher technology use (Vannatta \& Fordham, 2004). Levin and Wadmany (2008) observed teacher attitudes about technology change over the course of their intensive three year training program. While Levin and Wadmany’s longitudinal study 
examined the relationships among factors affecting teachers' use of instructional technology, the study itself was conducted while the teachers were participating in a special program where they were intentionally immersed in learning and technical support. We also need to study the relationship among factors while teachers are experiencing more typical day to day activities within their context. It is the teacher working within their normal educational setting where rich information can be found regarding the use of teachers' use of instructional technology tools. Teachers' situational context and personal influences combine to affect their instructional practices.

Complex interactions among dimensions and learners. Complex interactions exist among the learners, dimensions, and filters within the context (Levin \& Wadmany, 2008; Luckin, 2008; Luckin, 2010; Zhao \& Frank, 2003). A key factor is the strong, dynamic interaction between external influences such as school organization and culture with the internal cognitive and affective influences of each individual teacher (Levin \& Wadmany, 2008). In order to increase facilitative filters and reduce barrier filters to instructional technology use, we must develop a better understanding of how these complex interactions combine to affect teachers' instructional use of technology tools. It is an interactive relationship combining the personal factors from the individual with each of the four dimensions comprising the situational context. Examining it within Luckin's (2010) ecology of resources framework allowed me to focus on teacher's use of technology in relation to the context of teaching rather than in isolation. From a sociocultural perspective, intrinsic and extrinsic factors cannot be separated but rather should be examined together because it's about how historical, cultural and institutional factors combine to impact learners (Wertsch, 1991). 
Traditionally, students are the learners situated at the center of the ecology of resources framework, with the dimensions impacting them via a one-way relationship (Luckin, 2008). Learners who can exert influence on their educational context are capable of a two-way relationship between themselves and the dimensions (Zhao \& Frank, 2003; Luckin, 2008). Ertmer et al. (2012) found that teachers who use technology extensively in their instruction still experienced external barriers to technology, but they found ways to work around them, thereby overcoming the barriers, or minimizing those barriers. Teachers are capable of exerting influence regarding the educational context, engaging in a two-way relationship within this framework (Levin \& Wadmany, 2008; Li, 2010; Luckin, 2008; Luckin, 2010). Figure 1 shows sociocultural learning theory (John-Steiner \& Mahn, 1996) and the ecology of resources framework (Luckin, 2010) in its entirety. It illustrates the interactions among the dimensions with the teacher as learner, capable of exerting two-way influences among factors.

\section{Figure 1.}

Sociocultural Learning and the Ecology of Resources Framework.

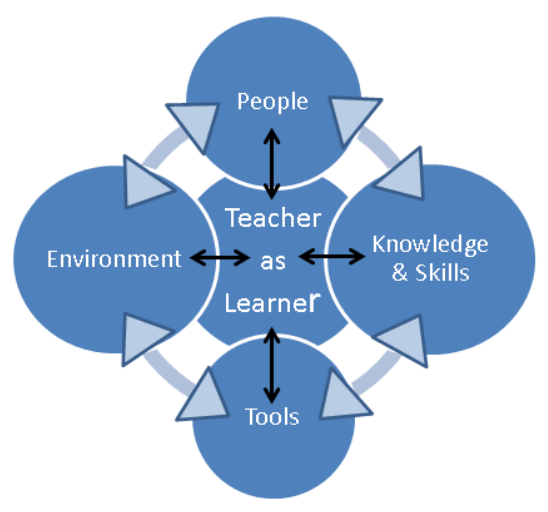

The two way relationship of factors in the sociocultural learning and ecology of resources framework aligns with the cyclical nature of the learning process within sociocultural theory (John-Steiner \& Mahn, 1996; Levin \& Wadmany, 2008). The learning process, or changing 
instructional practices with technology, must be viewed as being continuous in nature.

Consistent with adult learning theory, teachers progress from needing high levels of mediation from external influences towards relying more independently on internal influences as part of their learning continuum (Levin \& Wadmany, 2008). Personal factors within each teacher provide additional filters affecting each dimension (Finger \& Houguet, 2009; Howard, 2011; Levin \& Wadmany, 2008; Petko, 2012). We must therefore holistically explore the entire teacher context as it relates to their learning and the use of instructional technology.

Personal readiness to confront change, as opposed to resistance to change, affects teacher use of instructional technology (Levin \& Wadmany, 2008). Teacher beliefs about technology vary between belief and practice (Ertmer, 2005; Levin \& Wadmany, 2008). Their attitudes about technology are significantly related to their use of it in the classroom (Guzey \& Roehrig, 2012; Mueller et al., 2008). Teacher perception of risk costs and analysis of resource benefits also have been found to affect the use of instructional technology (Howard, 2011; Petko, 2012). Some perceived benefits of instructional technology as identified by teachers (Hennessy et al., 2005) include improving production, supporting processes, enhancing the appeal of activities, and fostering student independence and peer support. The learner centric ecology of resources framework (Luckin, 2010) helps to offer deeper insights about teacher perceptions regarding technology use, further investigating their perceived risks and benefits and the complex relationship among their contextual factors. It also helps us increase our understanding about how we can reduce barrier filters and increase helpful filters within the teachers learning context.

\section{Summary}

As found in the diffusion of innovation and adult learning literature, successful teacher learning and change must be voluntary, active, and collaborative in nature while occurring within 
an environment of social support and trust. Ideally, it is driven by need and individualized as much as possible to account for idiosyncrasies. As professionals, teachers are capable of making choices within their professional context. By combining major concepts from sociocultural learning theory (Lantolf \& Appel, 1994; John-Steiner \& Mahn, 1996) and Luckin’s (2010) ecology of resources framework, I explored teachers' cyclical nature of learning to use technology instructionally, while considering the teachers' context and the relationships among the dimensions and filters affecting it.

Teachers need to be able to reach and engage students within their digital environment (Luckin et al., 2012). Luckin et al. posit many researchers have studied factors affecting teachers' use of instructional technology, but few have situated teachers at the center of the context. Grounding this study within sociocultural learning theory (Mahn \& John-Steiner, 2002) helps to provide a deeper, more nuanced understanding of teacher experiences and beliefs related to the use of laptops in their science classrooms. Investigating teacher perspectives about instructional technology use as a continuous process within the ecology of resources framework allows for a more complete understanding of the factors within the teachers' personal contexts, as well as how they interact within the larger situational context (Mahn \& John-Steiner, 2002). “The development of a skilled, reflective technology-using teacher is a complex process” (Vannatta \& Fordham, 2004, p. 262), requiring a more specific, integrated examination of factors. Previous studies focused on stages of change or levels of technology use do not account for the cyclical nature of learning (Levin \& Wadmany, 2008). We need more studies examining instructional technology use as a continual process of mediation and internalization of learning (Levin \& Wadmany, 2008; Zhao \& Frank, 2003) in order to identify optimal approaches to facilitating teacher involvement in planning for technology and its use (Kotrilk \& Redman, 
2009). We also need to better understand how to help facilitate change in our schools and to develop teacher pedagogy related to instructional technology use (Fullan, 2012). Digital technologies offer opportunities to transform practice. However, new technologies themselves cannot improve learning for students (Luckin et al., 2012). When combined with pedagogy and content knowledge, technology tools can allow educators to personalize the context for learners, and help to integrate resources within time and space (Ertmer, 1999; Fullan, 2012).

In the following chapter I outline my research design and methods for investigating teacher use of instructional technology. This research was guided by major concepts from sociocultural learning theory and the ecology of resources framework, after building on concepts from the diffusion of innovation and adult learning literature. This study investigated the combination of the personal and situational influences on teacher instructional technology use. While this study focused on high school science teachers' whose classrooms received additional laptops, the term technology will be used synonymously to represent computers, laptops and technology tools in general. By considering the filters affecting teacher technology use, we can increase our understanding about how school leaders can most effectively address change in practices. Our schools need to experience an increased return on investment from the large sums of money being spent on technology (Cuban, 2001; Johnson, 2011). As Levin and Wadmany (2008) posit, it is time for school leaders and teachers to view technology as an integral tool for improving the learning environment. 


\section{Chapter 3: Research Design and Methodology}

The superintendent of the Midwestern school district examined in this study is placing an increased emphasis on digital electronic resources. In this district, printed textbooks are no longer purchased when it is time to replace them (personal communication, October 2, 2012). Consequently, large sums of money are available for the purchase of additional technology tools. The science coordinator was able to use these funds to acquire enough laptops for high school science teachers to achieve a two student to one device ratio in their classrooms, costing approximately a quarter million dollars (personal communication, December 10, 2012).

However, school district leaders have observed a wide variance among teachers’ instructional use of the laptops. Teacher use of the devices for instruction has been sporadic, or non-existent, according to the chief academic leader for the school district (personal communication, November 7, 2012). Issues of access to devices are not affecting the high school science teachers and the barrier of access and need for students to travel to the technology have been eliminated. After providing increased access to devices in high school science classrooms, district leaders wanted to better understand the difference between teachers who frequently use the laptops for instruction, and those teachers who use them minimally, or not at all. They also wanted to know what they can do as leaders to encourage instructional use of the additional technology tools.

As I sought to help this Midwestern school district better understand science teachers’ perspectives and pedagogy regarding the additional laptops, I used this study to investigate a problem from practice. This chapter explains the research questions, design, and methodology central to this investigation. I describe the rationale for participant selection, procedures and strategies for data collection and analysis, and plans for ensuring trustworthiness. In relation to 
trustworthiness I share my plans for addressing credibility, dependability, transferability, and confirmability. Ultimately, the purpose of this study was to develop an understanding of teacher perspectives related to the instructional use of the additional laptops in their high school science classrooms.

\section{Purpose of the Study}

The purpose of this study was to explore, understand, and describe high school science teachers' views regarding the use of technology in their teaching practices. It involved an exploration of teacher views rather than evaluation of them. Our students live in an ever changing digital environment and our schools need to be able to help prepare them for life within this information rich digital environment (Diem, 2010; Fullan, 2012; Luckin et al., 2012; McLeod \& Lehmann, 2012). Thus, it is imperative to help educational leaders deepen their understanding of teachers as learners and how they can help to facilitate use of $21^{\text {st }}$ century instructional methods among teachers, using technology resources as tools. For purposes of this study, it is important to clarify the focus is on laptop use because high school science classrooms received them in place of new textbooks. Also, within this study, the terms laptops, computers, and technology are being used synonymously.

This study seeks to understand high school science teacher perspectives and pedagogy regarding instructional technology and identifies potential facilitative and barrier factors (Luckin et al., 2012) influencing teachers’ instructional use of the additional laptops. Based on the findings of my study, I was able to provide recommendations for school district and building leaders about how to address teachers' use of the additional technology resources, especially as the school district increases its investment in digital instructional resources which I discuss in detail in Chapter 5. 


\section{Research Questions}

The research questions that guided this study were:

1. How does the implementation of laptops in one high school's science classrooms affect teacher instructional practices?

2. What factors do teachers identify as facilitating or hindering their learning and instructional use of laptops in high school science classrooms?

\section{Research Design}

The nature of this research problem and research questions lends itself to a qualitative research design (Roberts, 2010). Additionally, the qualitative researcher's view of the world as being holistic and complex fits with the sociocultural theory (Lantolf \& Appel, 1994; Wang, 2003) and ecology of resources framework (Luckin, 2010) informing this study (Merriam, 2009; Stake, 2010). Consistent with the qualitative research perspective, I am interested in the meanings people attach to their world (Denzin \& Lincoln, 2011; Merriam, 2009; Stake, 2010; Yin, 2014). Qualitative research facilitates studying the intricacies of social interactions within contexts (Merriam, 2009). As Merriam (2009) states, qualitative research is about the lived experiences of individuals and it is being used increasingly within the education field.

This study provides an in-depth investigation of a problem related to educational practice. By utilizing a qualitative case study, I sought to develop a deeper understanding from a holistic conceptual framework to address a problem from practice. My research addressed a current issue within a specific context related to teachers' use of instructional technology and the examination of teachers as learners. In seeking to maximize understanding of others' point of view, this research is subjective, interpretive, and situation-dependent (Stake, 2010). According to Stake (2010), the subjective nature of qualitative research can be simultaneously an asset and a 
detriment if proper methods are not taken to insure accurate observations and interpretations. Therefore, I must be explicit and formal in describing my research methods and procedures (Yin, 2014).

I employed a single case study method to investigate the complex social phenomena (Yin, 2014) regarding teacher use and non-use of the additional laptops as tools to transform their instructional practices for our $21^{\text {st }}$ century learners. A single case study was used for this study because it was focused on the science department as one unit within the high school. It is a method designed for gathering data related to multiple perspectives from multiple people (Stake, 2010). A case study is an ideal design to enable moving beyond previous research studies focused on classifying types or levels of technology use by teachers, or those describing characteristics of teachers who use instructional technology. It is the method best suited to investigating factors affecting instructional technology use, and for exploring the complex relationship among factors central to the teacher.

Because a wide range of laptop use by teachers had been documented, it was important to gather data from multiple teacher perspectives rather than designing a qualitative study focused on just one individual. According to Yin (2014), a case study design enables the examination of events and decisions too complex for survey or experiment methods. Additionally, a single case study design is appropriate for studying common, day to day events within their real world context related to people’s activity and practice (Yin, 2014). In this case, I expected to work with the teacher participants to bring their perspectives to light and to create understanding (Hatch, 2002).

As Levin and Wadmany (2008) posit, teachers are professionals who are critical thinkers and active constructors of knowledge. According to Argyris and Schon (1974), individuals learn 
from their experiences which result from their personal construction. I approached this study from the constructivist paradigm (Guba, 1990). Through constructivism, the aim of inquiry is creating understanding and authenticity as the researcher explores the realities existing within each person’s unique mental framework (Denzin \& Lincoln, 2011; Guba, 1990). Knowledge is socially and experientially based and local in nature, consistent with the sociocultural learning theory guiding this study. As the researcher, I partnered with the participants to bring their multiple realities to light as I worked to uncover patterns, noting similarities and differences among them.

When qualitative research is approached from the constructivist paradigm, theory serves as a way to focus the research, providing a way to record patterns from the observable data (Grix, 2004; Guba, 2009; Stake, 2010; Yin, 2014). Within this paradigm, conclusions emerge from the data in the form of patterns and relationships. They are often represented as part of the end point of the research after evolving from the data. As part of the research design process within this paradigm, I anticipated rival explanations within the data, choosing collection tools to assist with addressing them (Yin, 2014). The strategies must be geared towards triangulation of multiple methods in order to uncover and document different perspectives found within the research context (Denzin \& Lincoln, 2011; Merriam, 2009; Stake, 2010; Yin, 2014).

\section{Participants and Setting}

I selected the teacher participants based on their having received the additional laptops for use in their high school science classrooms. Previously, these teachers had no computing devices available within their classrooms. However, they received enough laptops to achieve a two student to one device ratio in lieu of new textbooks, thereby increasing reliance on digital resources. The high school science teachers have had convenient, ready access to laptops for 
their students for more than one school year. It was important to hear their thoughts about having and using the additional devices with their students, and about how they view their own learning in relation to making this change.

There are fifteen science teachers working in the high school I studied, teaching a variety of courses within the science discipline. The district science leader supervises the implementation of the science curriculum in conjunction with the science department chair for the school. Teachers provide instruction for basic, honors, and advanced placement courses. The high school principal hires and supervises the teachers in partnership with the school's leadership team of five individuals. The science department is one of fifteen curricular departments within this large, comprehensive high school. The school is known for its high level of academic achievement as evidenced by the large number of Presidential Scholars they have produced.

I chose this school setting because the science teachers had at least one full year of experience with the additional laptops and because district leaders have observed a wide range of use regarding the laptops. Although the school district has more than one comprehensive high school, and all high school science classrooms received the additional laptops, I intentionally chose to study science teachers from only one high school setting in order to allow for a more indepth investigation and understanding of their perspectives.

The setting for this study was a large high school located within a Midwestern college town experiencing constant population growth. The school district's enrollment increases by approximately two percent annually (personal communication, February, 6, 2014). At the time of the study, the high school's enrollment was over 1,600 students, according to its web site, and the school district enrollment is just over 18,000 students. It is a school district experiencing 
growth and change, especially as the superintendent has said funds should be directed towards digital resources when it is time to acquire new ones (personal communication, October 2, 2012). The school itself experienced change by adding ninth grade students the year of the study. As a result, there were some staff changes within the science department as teachers added ninth grade level courses to their science offerings. It was important to collect data in order to understand how all of the changes and dynamics within this Midwestern high school setting combined to influence the teachers' context and instructional practices.

\section{Data Collection}

As Denzin and Lincoln (2011) state, “...there is no clear window into the inner life of an individual” (p. 12). Therefore, using a collection of methods helped make the investigation more understandable and relatable for others (Stake, 2010; Yin, 2014). The multiple methods allowed for triangulation of teacher perspectives (Stake, 2010; Yin, 2014). For this case study, my primary data collection strategy was interviews using open ended questions consistent with my constructivist perspective. I listened closely in order to understand participant perspectives as clearly as possible and to build rapport with the interviewees (Hatch, 2002). Also, I asked clarifying questions when it was important to further my understanding of what was being said. I utilized classroom observations to corroborate the interview data and to provide additional insights regarding science teachers' use of the additional laptops. As part of my data collection strategy, I detailed my collection procedures and my plans for protecting my participants’ privacy. Finally, I used member checking and ongoing dialogue with the participants to further corroborate my interpretation of teacher perspectives.

Data collection tools. The goal for choosing data collection tools for a case study is to select multiple tools to gather evidence from multiple sources (Denzin \& Lincoln, 2011; Stake, 
2010; Yin, 2014). According to Yin (2014), there can be four main sources of tools for gathering data. They include reviewing artifacts and documentation, interviews, direct observations, and participant observations. For this study and its research questions, the teachers were the best source of data. Therefore, I chose the tools for this study based on which ones were most likely to provide the insights needed for me to obtain rich descriptions of teacher perspectives and pedagogy (Merriam, 2009). Using interviews and observations interactively to investigate the teachers' perspectives allowed me to get closer to the participant's point of view than other methods may have allowed (Denzin \& Lincoln, 2011). They facilitated my interacting with multiple participants as together we explored answers to the research questions.

Interviews. I performed one semi-structured interview with the seven science teachers who received the additional laptops and agreed to participate in the study. I followed the interview protocol I established (see Appendix A), which included background questions followed by more general, open-ended questions to allow for the interviewees to tell their story (Stake, 2010). The protocol also includes descriptive and structural interview questions (Creswell, 2009; Weiss, 1994) in order to investigate their perceptions about technology use. The descriptive questions were designed to explore participants' knowledge, experience, opinions, values, and feelings (Yin, 2014). Some questions were guided by suggestions from Hall and Hord (2011) in order to help solicit more detailed responses. Other interview questions were designed to further investigate findings from the research literature related to this study.

In developing and organizing the interview questions, I followed the dimensions from the ecology of resources framework (Luckin, 2010) and sociocultural learning theory (Lantolf \& Appel, 1994, Wang, 2003). The dimensions of people, tools, knowledge and skills, the environment, and the learner informed the interview questions because each dimension affects 
both the learning and work context for the teacher. It was important to hear their perspectives in relation to each dimension. When teachers shared the names of individuals, I substituted pseudonyms within the findings. I collected the data with the goal of identifying the filters within each of the dimensions, seeking to understand the interactions among the dimensions and the teacher learner.

The questions I framed around these dimensions served as a guide during the interview (Stake, 2010). I asked clarifying questions in response to the information each teacher shared in order to verify meaning and develop an understanding of the person's perspective as specifically as possible. I followed the semi-structured guide as much as possible to avoid reflexivity as described by Yin (2014) where a participant's answers or my questions may unintentionally influence one another. When interviewing, it is important to avoid leading questions (Yin, 2014). As Yin (2014) states, if the interview is performed as a guided conversation in an unbiased manner, utilizing questions such as "how" and "what" rather than "why", the interview can provide valuable perspectives. Before finalizing and using the interview questions, I field tested them with three teacher-colleagues of mine who were not part of the study. I also used these field tests as opportunities to practice my interview technique (Stake, 2010).

Each interview was approximately one hour in length. I conducted them face-to-face as one-on-one interviews in order to facilitate as much open and honest sharing from the participating teachers as possible. I audio taped each interview and transcribed the text from the interviews verbatim as soon after the interviews as possible. I also recorded my own reflections from the interviews immediately following them. Utilizing reflection notes helped me to 
understand how my own bias as a researcher might also have affected my interpretation and collection of qualitative data. These notes served as part of my research log, which was a valuable tool for documenting my research process (Stake, 2010).

Observations. As part of my research process, I performed two scheduled observations in each participating teacher's classroom during the spring semester of one school year. The goal was to use the classroom observations to corroborate information from the teacher interviews and to provide for triangulation of methods (Merriam, 2009). Observations are an important data collection tool because a case study is about everyday life and observing it directly is an essential component (Denzin \& Lincoln, 2011). Additionally, during my observations I monitored what I was observing, making sure it helped to support the themes emerging from the interview data. If it had not served that purpose, I would have modified my observation guide (Stake, 2010).

The semi-structured observation guide and questions served to orient and focus the classroom observations as outlined by Emerson, Fretz and Shaw (2011). As they posit, the researcher's point of view plays a key role in how observations are perceived and interpreted. During the observations I collected field notes using Wolfinger's (2002) salience hierarchy strategy of paying attention to what was most noteworthy and interesting. My observation protocol (see Appendix B) aligns with the ecology of resources framework (Luckin, 2010) which informed this study. It is focused around Luckin's four dimensions because it is important to witness how the possible filters within each dimension affect teachers' instructional use of the laptops. It included the teacher as learner to incorporate the sociocultural learning theory (Lantolf \& Appel, 1994; Wang, 2003) aspect of my conceptual framework.

While performing the observations, my objective was to remain as unobtrusive as possible to minimize interference and disruption of the instruction (Emerson, Fretz \& Shaw, 2011; Hatch, 
2002). I followed each observation by a conversation with the teacher in order to address any clarifying questions either of us may have had. I also recorded reflection notes about the observation as part of my research log (Stake, 2010). Throughout the observations I remained open minded as not all of my field note data may have seemed relevant on its own at the time. My field notes may have become relevant as I was analyzing the observations and combining the data with other data as part of my analysis process (Stake, 2010).

\section{Data Analysis}

I started data analysis early so it could inform my case study as it progressed. As part of an emergent approach to data analysis, interview questions may change as interviews transpire and the researcher seeks continuous improvement (Creswell, 2009). The goal with early and ongoing analysis, according to Creswell, is to have the opportunity to refine interview or observation protocols for improvement, making it a continual process of reflection and interpretation. Merriam (2009) describes qualitative analysis as moving between deduction and induction as part of the overall investigation. My aim for this research process was to pair analysis with data collection as closely as possible. I stored all data related to this study electronically and used a combination of manual analysis and computer software to store, analyze, and create audit logs of my analysis process.

According to Hatch (2002), “Data analysis is a systematic search for meaning.” (p. 148). Roberts (2010) says it is important to get a sense of the whole. I strove to make sense of all data as I transcribed my interviews and observation field notes. By combining findings from both the interviews and observations together during the analysis process, I achieved better triangulation of methods (Yin, 2014). Stake (2010) describes data analysis as a process of analysis and then synthesis where first the data is deconstructed and then reconstructed. I initially used a deductive 
typological analysis approach followed by inductive data analysis as I sought to move from specific ideas to general themes in the data (Creswell, 2009; Hatch, 2002). I identified similarities, discovering connections among the ideas, and ultimately building patterns as I constructed understandings in relation to my research questions.

To answer my research questions, I started by transcribing the recorded interviews myself, with this transcription process serving as my first reading and immersion into the data (Ravitch \& Riggan, 2012). I checked each transcription for errors by reading it while listening to the audio recording. Following advice from Ravitch and Riggan (2012), I noted contextualization cues such as pauses during responses or statements made with emphasis. Then I read and organized all of the transcribed data and field notes according to Luckin's (2010) general categories of facilitative and barrier filters, color coding the data according to Luckin's four dimensions of people, tools, knowledge and skills, and environment. There was also a category for concepts related to the teacher as learner perspective. My deconstruction of the data from the interview questions and observations was facilitated by their alignment with the framework's dimensions. This coding scheme served as the primary method for sorting and classifying the data (Grix, 2004). I consistently made sure all data I collected was coded, understanding as I coded new data, previous coding may have needed to be recoded (Stake, 2010). I sorted out the data and classified like items together to allow for themes to emerge.

After classifying and reclassifying the data, I reassembled the data to synthesize it into themes, seeking to answer my research questions (Stake, 2010). According to Stake, naming the themes with descriptive words would be a valuable part of this process, helping to focus the findings into patterns and also providing opportunities to uncover discrepancies. This part of my analysis process helped to develop themes around my research questions as I searched for and 
crystalized relationships among the information and categories. Merriam (1998) describes it as an inductive building process, while Denzin and Lincoln (2011) use the analogy of quilt making to describe this building process. An important component of this building process is to explore and address rival explanations (Yin, 2014). According to Yin, the more I can address and reject these rival explanations, the stronger my findings would be. As I described emerging themes and made sense of them, I used quotations as supporting evidence. I documented major assertions and conclusions with multiple forms of evidence (Stake, 2010; Yin, 2014). According to Yin (2014), it is vital to describe the themes and evidence so the reader of the study can experience them. Once I had descriptive conclusions, I had a peer review them to make sure I had interpreted meanings correctly. This process continued until my research questions were answered.

\section{Positionality}

My research perspective was from the constructivist paradigm. Multiple realities exist and together the researcher and participant can construct understandings (Stake, 2010), developing deeper understandings about their perspectives. Important individual perspectives exist as a result of unique experiences, and as a researcher I want to uncover and understand them from an empathic point of view (Stake, 2010). Additionally, as a qualitative researcher performing a case study, I also wanted to bring a degree of sensitivity to my interactions with the participants as I sought to develop a more nuanced understanding of their perspectives. Applying concepts of sociocultural learning theory (Lantolf \& Appel, 1994; Wang, 2003) with the ecology of resources framework (Luckin, 2010) enabled a more nuanced understanding of social interactions among teachers as learners, and the complex interactions among learners, dimensions, and filters (Friedrich \& Hron, 2011; Levin \& Wadmany, 2008; Li, 2010; Luckin, 
2010). This study presented an opportunity for teachers to think about the process of their own learning as it relates to their instructional practices. I had the opportunity to gather their multiple perspectives as part of my data collection process.

From the data I collected, I expected findings to emerge in the form of patterns and relationships (Denzin \& Lincoln, 2011; Grix, 2004; Merriam, 2009; Stake, 2010; Yin, 2014). My interpretations of the interview and observation data cannot be separated from my 20 years of experience with instructional technology (Denzin \& Lincoln, 2011; Hatch, 2002). As I interviewed the teachers, it was vital for me to clarify my role as a technology leader and to address and identify possible bias as a result of that role. Due to my associations with the school district, it was even more essential I emphasize to participants my desire to genuinely understand and describe their viewpoints as I was guided by the conceptual framework informing this research. I listened carefully and maintained an open mind at all times. I attuned to the context of the ecology of resources framework (Luckin, 2010) and sociocultural learning theory (Lantolf \& Appel, 1994; Wang, 2003) as opposed to my role in the school district. Ideally, my perspective as a technology leader benefitted how I attended to the data I collected, as well as to my analysis of it, because I remained conscientiously aware of my researcher bias and how it could affect both my research and the participants (Stake, 2010).

\section{Human Subjects' Protection and Other Ethical Considerations}

As a researcher working with human subjects, it was vital I adhere to all policies outlined by the University of Missouri Institutional Review Board (IRB), as well as the human subjects review process. It is of utmost importance that I protected the privacy of all research participants of my study and the confidentiality of the data I collected. As Fink (2009) states, my responsibility was to safeguard the information I was entrusted with by the participants. 
Therefore, wherever specific names were shared, I substituted pseudonyms. It is a primary ethical responsibility. Additionally, it was my obligation as a researcher to ensure this research had been reviewed and approved by the IRB, thereby demonstrating my compliance and adherence to the board's standards for conducting research with human subjects.

As part of working with human subjects and meeting IRB standards, it was vital I provided an informed consent letter for my participants to keep for their records, and had each person sign a participant consent form for my records, stating the ways in which their rights would be protected. These documents outlined key points such as participation is voluntary, it may be withdrawn at any time, and participants have the right to review transcripts. Also, as outlined by Hatch (2002), the informed consent document specified if any potential harm or discomfort may result from participation in the research study. I was also very clear about any potential conflict of interest I as the researcher may have had in relation to the study.

Additionally, as I was representing the University of Missouri as a doctoral student researcher, it was imperative I abide by ethics guidelines and display ethical behavior at all times, especially as it relates to intellectual property and plagiarism. The American Education Research Association's (AERA) Code of Ethics (2011) provides guidelines for research ethics related to scholarly and professional standards, misuse of expertise, non-exploitation, and competence, in addition to all of the considerations I have already presented. With all of these considerations and guidelines in mind, I remained diligently aware of protecting human subjects and all ethical considerations as a qualitative researcher.

\section{Trustworthiness}

Lincoln and Guba (1985) recommend qualitative results be evaluated using the standard of "trustworthiness," established by credibility and confirmability. My data includes the words 
reflecting the participants' opinions, thoughts, perceptions, and feelings. It is important I interpreted and described them accurately (Roberts, 2010). My data collection process and analysis procedures were designed to provide the assurance they were performed as objectively as possible (Bryant, 2004). Combined, they provide the "credibility factor" (Roberts, 2010, p. 161) needed for qualitative research.

Credibility. For this research study I utilized multiple data collection methods and repeated observations to insure credibility and dependability. It was important I checked the accuracy of my findings by employing many procedures. I had a peer review the themes and conclusions I developed. I have shared any data that ran counter to the themes, and used quotations to support the themes I identified. I also checked all interview transcripts for mistakes. My use of member checking and ongoing dialogue with participants also served to increase the credibility and trustworthiness of the data. My participant consent form informed the teacher interviewees of their rights as participants in this case study, while emphasizing how I would maintain confidentiality.

Dependability. I transcribed the interviews soon after conducting them. I also coded the data I transcribed as I completed each transcription, using typological analysis (Hatch, 2002). I organized my coding around the ecology of resources dimensions (Luckin, 2010) which helped frame this study, allowing me to analyze the filters evidenced within each of the dimensions and understand their effect on teachers' instructional use of the laptops. Along with the teacher as learner, they include people, tools, knowledge and skills, and the environment. The patterns I found in my coding allowed me to establish themes to answer my research questions. Remaining open and adaptive to new information throughout this process insured I was considering all viewpoints and possible conclusions while working to document different perspectives (Denzin 
\& Lincoln, 2011; Stake, 2010; Yin, 2014). My use of audio recordings from the interviews and my recording of the code definitions I used further enhanced the dependability of my qualitative data.

Transferability. I openly discuss any contradictory data evidence. My use of thick, rich descriptions promotes credibility, dependability, and transferability (Creswell, 2009). By providing the reader with as much detail as possible and bringing forth the voices of my participants, it was my goal to allow others to more successfully determine what elements of my study may be relevant and applicable to their own setting or situation. This approach is especially vital because I was performing a single case study limited to one school setting.

Confirmability. I have maintained careful records of all information related to my research under lock and key, including interview protocols, interview transcripts, observation guides and notes, researcher field notes, and researcher reflections. I kept a log of my activity as recommended by Stake (2010). All of my paper copies have been kept in a locked container, and I password protected all electronic transcripts and notes.

\section{Limitations}

As Hatch (2002) posits, "Participants are the ultimate gatekeepers” (p. 51). The researcher must earn the trust of their participants. My assumptions as a researcher and the manner in which the participants chose to involve themselves and share information could influence limitations of a research study, and ultimately, the quality of the study.

This study had several limitations. The design of this case study was somewhat limited because eight of the science teachers within the high school choose not to participate. I made every attempt to encourage teacher participation. Because the science coordinator for the school 
district supported this study, I asked the leader to encourage teachers to participate. In an attempt to prevent any possible limitations related to the interview settings, I offered the participating teachers the opportunity to select the interview location.

The quality of teacher participation and sharing may have been limited by the degree of trust I was able to develop with each interviewee. A limitation may also have resulted if the participants being interviewed did not share information openly and honestly with me as the researcher. Also, the quality of teacher interview participation may have varied depending on situational influences such as how they may have been feeling on the day of the scheduled interview, or how things had recently been going in their classrooms.

In relation to the classroom observations, they may have been limited by my inability to attend to everything occurring in the room simultaneously, as one observer of many. I used my observation protocol to guide my focus and attention. Also, my presence in the classroom could have inhibited or altered the observable data and participant behaviors. Having a researcher in the room taking notes may have been seen as intrusive, even though I tried to remain as unobtrusive as possible.

Additionally, the potential for researcher bias exists because I have worked with the school district involved in this study. And finally, with this study being limited to only one school site, its relevance to other sites will depend on how closely others can identify with it. I have included as many details as possible in order to offer increased opportunities for others to find similarities with their own sites. 


\section{Conclusion}

This study employed a single case study methodology to develop a deeper understanding of teacher views regarding the use of technology in their teaching practices. By utilizing a holistic context within the ecology of resources framework (Luckin, 2008) situated within sociocultural learning theory (Lantolf \& Appel, 1994; Wang, 2003), it allowed for a more nuanced understanding of teacher perspectives related to use of the additional laptops in high school science classrooms.

High school science teachers from one Midwestern high school were invited to participate in this study because they received additional laptops instead of new textbooks for their classrooms. I corroborated individual interviews with classroom observations as my data collection strategies. I analyzed my data with a typological approach, combining deductive and inductive coding methods to identify themes and relationships among patterns (Creswell, 2009; Hatch, 2002).

My findings could help leaders from one Midwestern school district to develop a better understanding of why some teachers embrace using the additional laptops, while others do not. Additionally, as this study addresses a problem from practice, it helped these leaders understand what they can do to reduce potential barriers to teachers' use of the additional laptops. Finally, this qualitative case study contributes a new perspective to the body of literature related to investigating teachers’ use of instructional technology. 


\section{Chapter 4: Findings}

This exploratory, single case study investigated high school science teacher views about their use of laptops in their science classrooms and how having additional laptops has affected their instructional practices. A second purpose was to identify factors facilitating or hindering teacher learning about using the laptops instructionally. I designed the interview questions and observation protocol around the ecology of resources framework (Luckin, 2008) and sociocultural learning theory (Lantolf \& Appel, 1994; Wang, 2003). The goal was to put the teachers at the center of the context as learners. Seven of the 15 science teachers from a large Midwestern high school participated in this study during the spring semester of one school year. The participating teachers represent an average of approximately 22 years' teaching experience.

These veteran teachers voluntarily and willingly responded to my email invitation to participate. They openly shared their thoughts and ideas with me. This case study allowed me the opportunity to gain an up close, more in depth understanding of the high school science teachers' context. As I noted in my research journal, the classroom observations provided me with a better understanding and insight into each teacher's day-to-day instructional context. The observations did more than just corroborate the interviews. They helped me develop a more thorough understanding of each teacher's context, their teaching, and their students.

I analyzed the findings according to the ecology of resources’ four dimensions and around the teacher as learner. Additionally, within each dimension I identified the facilitative and barrier filters reported by the teachers in order to identify what factors were hindering or facilitating the use of the laptops. Following the ecology of resources framework (Luckin, 2008) 
and sociocultural learning theory (Lantolf \& Appel, 1994; Wang, 2003), I grouped my findings as themes within the four dimensions of people, tools, knowledge and skills, and the environment as well as around the teacher as learner at the center of the context.

\section{The People Dimension}

The people dimension includes all those who exert influence on the educational setting. Five main facilitative themes emerged within the people dimension. Interview data for the people dimension was consistent among the teachers for the people dimension. The following themes all support the conclusion the teachers need multiple people supporting them in the use of the laptops, and they are not necessarily technical support staff, although those staff members play a vital role in support of teachers using laptops instructionally. The five main themes include: (1) technical support staff members are helpful, (2) colleagues and students help with strategies regarding laptop use, (3) collaboration facilitates successful use of the laptops, (4) expectations are implied through provision of resources to support laptop use, and (5) professional learning teams (PLTs) are instrumental in facilitating laptop instructional use.

Technical support staff members are helpful. Teachers reported feeling supported by both district and building level staff in their use of the laptops. Overall, their building technician and media center staff were praised for timely response, despite teachers experiencing issues with being able to log in to the network, or other hardware type issues. During the observations I witnessed both building and district level technical support staff working at the high school. Additionally, when the Internet went down during one observation, the district network staff had it back up within three minutes time. As teacher one shared they are, “...very willing to do whatever they can do to help us, so as we had login issues or this or that, you know, we'd go find whoever could help us, and that sort of thing.” Teacher two said, “There’s been a number of 
ways that people have helped, uh, between our media specialists who have helped with the hardware issues, login issues, those kinds of things.” Teacher three was also in agreement sharing, "And through our media specialists and so forth, the technology is, is good. And, and I think it's focused on what do the teachers need and let's see how we can get it there.”

The teachers feel they have the support and resources they need from the technical staff, even if it requires the staff to investigate answers and solutions for them. According to teacher four, “...we've got resources in people. Joseph (a media specialist) and Megan (the building computer technician) both are excellent. Um, if they don't know the answer, they'll get it, get on it...I wouldn’t say that people present barriers anymore.” Teacher five indicated the support from both building level and district level staff members has been very helpful:

I find that the folks here in the support part of the school are wonderful, Mara and Joseph and Tammy are on my side and they keep an open conduit, you know, through Jane and the media center staff that, you know, we need something, I mean usually before I'm off the phone with June, Joseph is in the room, you know, helping me out with it or giving me feedback or bringing up an extra machine if one of them goes kaput or something. So I've found that I've had a lot of support as far as building level, and of course Tom has been really helpful and Mark has been really helpful in terms of providing kind of those outside resources and access (from the district level IT staff) and working out glitches. So that aspect I've been real pleased with. It's gotten better. (Teacher 5)

In addition to a feeling of overall support from technical staff, one teacher also mentioned having an improved relationship with the technical staff, especially from the district support staff, “And I think there's a better relationship now than I would say even five years ago. It was very caustic." Along with this more positive, supportive relationship, the willingness of the technical staff to investigate solutions and to be readily available to the teachers supports the conclusion teachers report only helpful factors related to the technical support staff helping them with their use of the laptops, especially despite experiencing login issues and setbacks related to their use of the laptops. 


\section{Colleagues and students help with strategies regarding laptop use. Many of the}

teachers shared descriptions of how their fellow teachers helped provide ideas, strategies, and

tips about using the laptops:

And so it did take more of our time to do that (use the laptops) because learning about the process [laughs] and oh my gosh, this isn't working, then what can we do to fix it, figuring out that fix...but again, with that people piece of “Oh my gosh, don't do it like this, log on to your computer as guest”, you know passing that information along has really helped. (Teacher 2)

Teachers being confident in knowing colleagues can be relied upon to help with troubleshooting strategies is an important component of their being willing to use the laptops instructionally. For teachers who view using them as a risk, knowing your peers are there to help could be very reassuring. Additionally, receiving tips from other teachers helps to avoid pitfalls others may have experienced. According to teacher three:

Show me again how to do that? Cause it's just like teaching. It's that one on one piece. Okay, you did the, the big presentation how to do it. Now a teacher can walk around and go, oh, I see where you got stuck. Let's, let's fix that.

This teacher also shared how students help with the process, “And, and those kids teach me. So

I've learned quite a bit and I'm learning quite a bit to deal with this.” The experience and

knowledge about the laptops students demonstrate can be helpful to teachers when it comes to figuring out how to perform specific skills. Teacher five shared:

As new things came available I would have the AP kids explore and do them 'cause they're just so bright and tech savvy, and then I would switch those into using them once I learned how to do them. I'd switch them to the kids. There's only so many hours in the day...I don't have to know how to do it. I could say, 'Make a Claymation video using iMovie.' It doesn't matter that I haven't practiced this and don't know how to do it because my AP kids will do it and they'll make these great projects [laughs] and I'll figure out how they do it by circulating around, and then I can do it with my general kids 'cause I'll learn from them. 
Teacher five has developed a way to empower students by having them figure out details of new programs and applications, thereby freeing the teacher to concentrate on teaching. Relying on students to help also means this teacher is okay with not being the only expert in the classroom and is okay admitting not knowing how to do everything. Teacher four echoes the same point stating:

Don't be afraid of it. Get help from your friends and colleagues who know how to use it and get help from the students. Don't be afraid to say to the kid, "Hey, I am a PC person. I'm not an Apple person. How do you do this on an Apple," you know, or vice versa.

During multiple classroom observations I witnessed students helping other students with using a program. I also observed them helping each other figure out how to have a program work on an iPad like it does on a laptop computer. Interestingly, I also observed students in a physics classroom debate the benefits of laptops vs iPads in a very knowledgeable manner, knowing exactly what they wanted each device to be able to do for them.

The value of students who quickly grasp how to do things with technology tools cannot be overlooked. Students can be a rich resource when it comes to using different technology devices and programs in the classroom. Interview and observation data show evidence of teachers helping other teachers with usage tips and suggestions, as well as having students help support technology. An important component of support is being willing to ask for help from other teachers and students. Having colleagues and students who are readily available in the education setting can enhance the formal support provided by technical staff members. Expertise for using the laptops them becomes more of a shared responsibility. In addition to having peers and students help with strategies regarding laptop use, the participating teachers reported collaboration with colleagues also had an impact on their use of the laptops. 
Collaboration among the teachers facilitates successful use of laptops. Teachers

shared several examples of how collaboration with their fellow science teachers has helped them

use the laptops instructionally. Teacher one shared, “So that helped because if one of us didn’t

remember how to do something, maybe another one did, and that was good.” Teacher two

described how helpful this collaboration had proven to be:

...by being able to put our heads together on it it's been I think incredibly powerful, and I've seen such huge differences in my classroom on that student achievement piece. So it's really been exciting on being able to do that... and by being able to share ideas and stuff, the products that we're able to come up with are so much better I think than anything that I could come up with on my own because of the time involved, you know? Yeah I could come up with something fantastic if I had the time to be able to put into it, but when it comes down to the realities you just don't have those, that time to make that many changes and incorporate things like the technology piece. And being able to come up with some things that again have been just magically successful, you know, the first time around rather than taking - if you're working on your own it seems that those things take several years almost to get to that point because of, oh wow, this didn't work or I could have this in - and you know by able to putting in that time over those years versus now, wow, we can get this all and get to a really good spot very quickly that first year.

Teachers also shared how collaborating about laptop use has helped them improve their

instruction in a more timely and relevant way than before:

What we do, Jessie is a day behind me right now....but what I do immediately is after I did something I'll walk through there and I'll say "Okay, I did this. This didn't work, this did work. When you do it tomorrow, have that already on a Word document in the secure folder so the kids can just click on it because, um, it took 'em too long to type this in or this link didn't work. So have a live link there. (Teacher 5)

And teacher five elaborated further on the more dynamic lesson improvement the collaboration around the laptop use has facilitated:

Don't bother with this. It was just too tedious. Nobody cared, but this other thing really caught their attention to use it. So a way that people can get, keep that as a dynamic developing lesson when you come up with a resource that's not just something that you put in a folder and stick there and say "Use this next time", but it's something that you've been able to put some feedback in and, um, indicated how well it went over with the kids - what sort of improvements needs to be made to it.... But there needs to be that kind of 
collaborative piece, and I would really focus on that as one of your quadrants is that collaboration within the group of teachers that are actually teaching the lessons. I think that's essential.

The teachers also reported sharing across grade levels within their scientific disciplines.

Teacher six shared:

All the physics teachers are teaching the same thing. All the biology teachers are teaching the same thing. Now they throw their own twists in it, but it's the same formative assessments, the same summative assessments, the same activities. And the chemistry teachers are doing the same thing. So it's really kind of exciting to, to see that.

The value of sharing with each specific content area within science was echoed by another teacher. “It's content area specific. The biggest collaboration is within each subject area. And then the Physical Science or Physics and Chemistry will interact some, especially because they'll use Logger Pro in the lab as well for data collection and graphing...”(Teacher 1). Another teacher shared:

We collaborate and we pretty well operate and use the same curriculum and the same objectives and the same activities...And different teachers in our group spend time and surfing around and trying to find different apps and different, you know, animations and different video clips and they share that. (Teacher 7)

And teacher seven also shared:

I mean, you can even incorporate some of the technology into the lessons that you're already doing. It's just a matter of replacing that glass thermometer with a probe that takes better data quicker and graphs it for you while it's taking it. I think if people that have them and aren't using them were shown ways that it is being used by other people, and the results that they're getting from it are encouraging, positive, you know, if they were shown that's it's really not gonna create a lot more work for you.

This teacher was indicating how teachers are the best ones to show other teachers ideas for using laptops instructionally. Other teachers also referred to one teacher in particular who presents a barrier to their collaboration regarding both using the laptops with students, as well as to adopting the modeling instructional approach many of them are using.

Well, all of my colleagues except for one are very much into the whole pedagogy modeling and using technology. Not just for the sake of technology, so it's not just okay, 
we have a computer so we're going to pull it out every single day. But when is it going to provide a good learning experience for the kids, or a good data collection experience in the lab. So it's going to add to what we're trying to do.....He has not incorporated them at all into the other sections of Honors Chemistry that he teaches. And he does not collaborate with me in the Honors. He does his thing, and I do my thing. (Teacher 1)

Teacher four shared:

It seems like everybody fairly well interacts and supports each other... There - there's really only one, Mr. Baker.... Yeah, so the kids - students in that class, Honors Chemistry, they aren't getting the full utilization of the modern world and they walk into other classes and come out of other classes and go to other classes and they do use the modern world.

Teacher seven also referred to this colleague serving as a barrier:

I think for the most part, the people that I work with are facilitators, they encourage and help. We help each other develop how we're gonna use them. There are, I guess, a few examples of maybe people who are more, uh, I use the, you know, the, the cliché 'old school' where they would prefer just to do it the way they've always done it, and, it's worked fine without using graphic animations for years, and "I don't see why I should have to change." There are some of those, but for the most part, people are pretty positive about using it (technology).

Other teachers elaborated on this same barrier, "But, but the bottom line is there are some individuals who are not comfortable and they won't ever do it. Ah, but, but the rest of the folks are willing to try and they're willing to learn and so forth” (Teacher 3). When I asked him how we might work to reduce the barrier teacher three replied, "Retire. That's the way it will change." Other teachers also expressed some indications of frustration with having a colleague as a barrier to collaboration:

The peer pressure's there. I personally have walked in and said, "Here's something he and I used with mercury," as in mercury - [laughs] in glass tubes that kids could spill out on a lab bench. And it's a software simulator that you can do the same type data collection, get the chemistry data, et cetera, and it's all software... And - and it's really good and so I started using it and I just simply showed it to him. I said, "Look, a lot of time you should use this, have to use this." I just said, "Look how cool this works and you can download the data into Logger Pro, you can print off the graph, and so yeah, you can still have them hand graph other stuff but this lab, you don't have to have them hand graph so they can get used to technology and then technology graphs for you." And I showed it all to him. I said, "You need help, I'll be glad to help you." "Thanks. Thanks a 
lot." Boom. [Laughs] That was it. It didn't go anywhere. ""Look. Here's the way I'm going to do this lab. Here's the computer. Here's whatever. I'll show you how to use it." "No, thanks, but I'll do it the other way." (Teacher 4)

The department chairperson explained the situation with the teacher who will not collaborate with the other teachers, explaining the teacher has it just the way the teacher wants instruction and therefore will not alter his instructional approaches:

There, there are a few that feel like that they've got it exactly the way they want it and it's the right way to do it. And they're good teachers. And they're really pushing the kids. So they don't change much. And there are others, like myself, who just keep feeling there are always better ideas out there. And you gotta keep your ears open and listen. (Teacher 3)

However, one teacher feels so passionately about this teacher refusing to collaborate, or adopt the modeling pedagogy, or use the laptops, the teacher contacted me via email a few weeks after our interview. This teacher shared test results from the American Chemical Society. The message said:

Jaimie taught her 3 sections with a Modeling approach. Baker taught his with a "pour it down your throat" traditional approach as I discussed in the transcript. Below are the results on a national, standardized, end of year exam from the American Chemical Society. The results say it all. Modeling students scored at the 68 percentile while the Traditional students scored at the 29 percentile. Modeling blows away the traditional teaching approach!! For his students' needs Mr. Baker must change how he teaches.” (Teacher 4, $6 / 27 / 14)$

The test results show the students who had the chemistry teacher using modeling outperformed the students who had the teacher using traditional instructional methods by a 39 percent difference. Table one below shows the results teacher four sent via email. While these test results reflect student performance from just within the chemistry discipline, the teacher felt strongly they illustrate how this teacher, who refuses to collaborate with colleagues, is serving as a barrier to teachers as well as to student performance. 
Table 1.

Comparison of Student Test Scores of Modeling vs Traditional Instruction

SUMMARY Modeling vs Traditional with National Ranking June

2014

\begin{tabular}{llll} 
\# Students & $\begin{array}{l}\text { 80 Question Exam } \\
\text { Mean }\end{array}$ & $\begin{array}{l}\text { ACS National Percentile Rank } \\
\text { 2011 Exam }\end{array}$ \\
\cline { 1 - 3 } 48 Modeling & $61 \%, 49$ out of 80 & 68 & Mean 42.5/80; STD 12.1 \\
68 Traditional & $44 \%, 35$ out of 80 & 29 &
\end{tabular}

Overall, the teachers reported most of their colleagues helped facilitate their use of the laptops. The only barrier filter to this helpful collaboration is when some colleagues refuse to collaborate with the other teachers. Teacher four summed up this point:

So everybody tends to try to incorporate, use it, help each other...Colleagues also, you know, other science teachers or whatever, really use technology for the most part. A few don't, but most of them do, so they help each other. So it's a real collegial, help.

Expectations are implied through provision of resources to support laptop use. The teachers consistently shared they had not received explicitly stated expectations from either their building or district leaders about using the laptops instructionally. Rather, they felt the support was demonstrated through leadership actions, support, and respect for their professional judgment. Teacher four shared:

I don't think anybody leadership-wise, like principal, or department chairs, or anybody, has specifically said, "We expect this. Now there's this underlying theme of, "We want you to be on board with technology. We want you to try to interact in a new way, you know, whatever, so the kids are experiencing more what they might experience in the real world,

or when they go get a job, or when they go to somebody else's class." So it's, um, not a concrete expectation, or a statement, or whatever - but it's just an underlying, "We're all trying to move forward.” 
Speaking in relation to leadership at the school level, teacher one shared:

I don't even know that they know other than if they've been in class when we've used them. Yeah, I think they're kind of detached from that side of things. Maybe because we're kind of compartmentalized and as a district, you know, between the subject matter the subject area divisions and then the fact that we have an I.T. division or department that takes care of those things, I don't know how much the administrators within the building really are involved in any of it, or if they have to think about it or not a part of their paradigm or job description.

Teacher three also expressed a perception of the building leadership being detached from the laptop implementation saying, “From a building level there really isn’t a push at all.” Teacher

five spoke about leadership at the building level as well as in relation to the district science coordinator. This teacher indicated since the laptops were in place of new textbooks, the purchase of them implied an expectation they would be used:

Well I know that as far as the school, I know that they expect us to be able to help students gain access to content, understand the content, be able to think and problem solve, develop science skills and mastery of what they need to know to meet curricular expectations as far as the school. Um, as far as the district, um, Mack has let us know that, you know, because of this initiative they didn't want us to use textbooks, that this was supposed to be our substitute for textbooks.

Teacher six also referenced implied support from both the building leadership and the science coordinator:

I don't think there's a directive that says you have to use the SMARTboard, you have to use technology, you have to use the laptops. I think that's - and that's a good thing..." "So from that standpoint I don't see much directive from Tanya or from Mack to do it. They are supportive of, of that." "But I think he allows us to explore what we want to. But and he'll, do his best to support it if he can get the funds for it. So, I don't see anybody pushing saying you have to use it.

The teachers all spoke about leadership and expectations from their science coordinator when they were asked the general question about leadership from the district level. The 
participating teachers view the science coordinator as their district level leader. It is both the knowledge and financial support from their coordinator the teachers view as support for their using laptops instructionally. Teacher four compared the coordinator to the building leaders:

He's kind of the same way. He probably pushes a little more. "Here's a cool app. You know, here's what another teacher found. Here's the - the National Science Teacher Association's conference we just got back from and here's ten things we saw. And so he's more definitive, "Here's examples. Here's something. Here's a website, you know. Check into this." So he definitely keeps in - in that direction.

Teacher three, who is the science department chairperson, touched on the financial support and provision of resources the science coordinator provides:

So he was there supporting us with what we wanted and needed. He was never saying you must use technology. He was saying what do you need? How can I help you get it? And, and that's where the push has been.

Teacher two also appreciates the support from the science coordinator in relation to the modeling approach and using the laptops:

He was very willing to facilitate that. As we saw that need he was willing to make that happen because he saw that as a very important piece to designing those lessons and again making that content more relevant and up-to-date for students. So, very much a huge facilitator on getting us that stuff and making it workable, so, including things like, you know, the charging carts for several of the rooms and stuff like that.

And teacher one concurred, explaining the expectations were not explicit, but rather implied through the science coordinator's actions:

Hmm. None specifically that he articulated, however, he knows that we're doing modeling curriculum and that modeling curriculum relies on the use of simulations and models and data collection. So I think he probably didn't have to specifically say anything because it was going to happen based on what we were going to be doing.

During their interviews, all of the participating teachers mentioned the modeling approach in conjunction with using the laptops. Their responses often combined the topics integrated together. Because the modeling approach so clearly requires technology tools, the lack of explicitly stated expectations does not seem to matter for the participating teachers who show 
evidence of using the laptops instructionally. However, one has to wonder if part of the reason the one chemistry teacher is permitted to ignore the modeling approach to instruction is due to the lack of clearly stated expectations.

Finally, one teacher shared thoughts about their science department chairperson when responding to the question about expectations from their school leadership:

He's, he's very good about being an advocate for us. He's not a top down kinda guy. He comes to our rooms and finds out, "What do you want?" He asks, "What do you want?" And then, so it's he, he finds out from us what we want, and then he does whatever he can to get it. (Teacher 7)

Teachers appreciate leaders respecting their professional judgment. No one is explicitly telling the teachers what to do. When talking about expectations from building and district leaders, many of the teachers elaborated on the respect they feel from their leaders.

We're given a lot of latitude. We pretty well get to choose, like, you know, we're not told, "Here, this is the textbook that your use - that you're to use, or this is the software that you're to use or these -" you know, we are able to go out and find whatever it is we wanna use, and then in some cases purchase licenses to, um, use software. (Teacher 7)

As the subject area expert, teacher seven values being able to make decisions about instructional resource purchases. Teacher one echoed the same message:

Paying attention to what the experts in that subject area say they need. You know, and you have to of course, know that these people know what they're talking about. And if you're not sure, then sitting down with them and really listening to why do you feel that.

Teacher one referred to the importance of being heard, and having a voice regarding decisions about instructional resources.

Offering advice about leadership expectations for the use of technology in general, teacher five shared:

School leaders try to, school leaders just need to try to get out of the way and let people explore and experiment and use it and not put a lot of guidelines, restrictions, um, oh gosh, please don't make us collect data and report back. That's the fastest way to kill enthusiasm 
for anything because then it becomes this tedious chore and you don't even wanna bother, but if they just get out of the way and let us do it.

The science teachers appreciated their building and district leaders' show of support rather than their mandating of expectations, or accountability measures. Teacher six offered this advice when asked about leadership expectations:

So I think there shouldn't be a directive that says you have to use laptops, you have to use iPads, you have to do this. But at the same point in time, those resources do need to be available and encouraged to use.

Encouragement, respect, and support from both district and building leaders is what the participating science teachers have experienced in relation to using the laptops with their science instruction. The teachers’ positive comments regarding the respect and support they feel indicates they feel their professional judgment is valued, thereby leading to a positive feeling of job satisfaction, although this aspect was not explored as part of this study. The teachers also want to be listened to, and feel they have input into decisions being made about their instructional resources.

\section{Professional learning teams (PLTs) are instrumental in facilitating laptop}

instructional use. Every teacher participating in this study discussed positive aspects of their PLT without any prompting during the interviews. The teachers described their professional learning teams, also known as professional learning communities, or PLCs, as being the strongest facilitating factor within the people dimension. Approximately two to five teachers participate in each PLT according to their specific discipline within the science department. The teams meet to plan for and evaluate student learning. Teachers described their team time as being valuable for enhancing their use of the laptops. Teacher three shared, "So that collegiality has just bonded., and made us both better instructors. And I think and really enhanced our use of the technology.” Teacher two shared: 
As far as ways to use them our PLT has just been really phenomenal as far as incorporating different things. As we are with science this year we're incorporating the new NGSS (Next Generation Science Standards) into our curriculum, and so we've really reworked the whole biology curriculum with the new NGSS standards in mind and within doing that, that technology piece I think has been a pretty big one. It's allowed us to incorporate several kinds of new key activities and stuff that have really made the content that we're teaching in biology more relevant to our students' lives.

The collaborative nature of the PLTs allows for teachers with technology knowledge and skills to work with their colleagues who may not possess the same level of skill. According to teacher four, “That's truly - truly collaborative and going, you know, that kind of doesn't work that way and, you know, you can tell me that and I can tell you that and everybody accepts each other.” And as teacher five shared:

I've got some experience on the electronic platform side of it and so I can help with that but I think giving people that collaborative time to work and develop stuff, not fake stuff but real stuff that they're gonna use, and it needs to be done within a PLT I think so that they can get feedback right away.

Teacher one shared frequently about how the modeling approach relies heavily on teachers using laptops instructionally. Teacher PLT time for this teacher involved becoming better at the, “modeling curriculum” (Teacher one), as well as using the laptops within that curriculum:

Well, within the PLT we definitely talk about the lessons, and we plan lessons together. And so especially with the modeling, but even before you know, so it'd be like whoever was the lead person. Oh, this didn't go very well and we did this. Or we need to change this example and we'll talk about it.

Other teachers indicated their PLT experience facilitates both improving their lessons, of which using the laptops is just a part of the tools they use:

Professional Learning Team meetings....for us to collaborate with members of our content area... to collaborate and the, some of those, some of that time is spent actually using the probes, doing the, doing the labs that the students are gonna do, so that we know, we have first-hand experience of what they're gonna have to do when they do the lab and what kind of expectations we should have for the data that they're going to be collecting, and, so, yeah, we have quite a bit of time to collaborate. (Teacher 7) 
And teacher five shared:

Um, our PLT is phenomenal and so we do all of our planning collaboratively for biology. Um, I don't have a PLT for AP, but I do have one online because I use the AP Central electronic discussion board and then I have a lot of side conversations with my friends from AP all over the country, so we basically have a virtual PLT.

The overall value of the PLT's effect on facilitating the use of laptops within the people dimension was emphasized repeatedly. The science department chair explained, "My main focus now is, is not evaluating teachers. It's to try and help the PLTs become more effective PLTs...And most are functioning at a really good level....and they're saying thank you for this.”

(Teacher 3). And teacher five shared this advice regarding how people can facilitate teachers using laptops instructionally:

So as you're looking forward to trying to help others incorporate more of this technology into the classroom, what has helped us immensely in science is having these PLT times where we've actually pulled out the stuff and worked on it and come up with things together.

\section{The Tools Dimension}

The tools dimension includes the physical artifacts and digital resources available to support learning. Within the tools dimension, eight themes emerged from the interview and observation data. Five of the themes relate to facilitative factors, and three of the themes consist of barrier factors related to hardware issues, compatibility issues, and students not having Internet access at home. The facilitative themes include (1) laptops improve overall delivery of instruction, (2) laptops eliminate the need to schedule access to computers, (3) laptops facilitate inquiry based and student centered instruction, (4) effective instruction is facilitated by print and electronic tools, and (5) the availability of multiple technology tools enables active learning.

Laptops improve overall delivery of instruction. The teachers shared how both the modeling approach to instruction and the implementation of the Next Generation Science 
Standards (NGSS) were important to their instruction. Having the additional laptops facilitates

both of these goals. Teacher six explained:

Looking at all that and the NGSS it's like this has modeling written all over it. So we jumped on that bandwagon in chemistry. And it's a philosophy of students understand how to model. And they do that modeling by active engagement....And but a lot of it was computer driven.

Teacher two also referred to how the laptops have facilitated implementation of the NGSS:

We're incorporating the new NGSS standards into our curriculum, and so we've really reworked the whole biology curriculum with the new NGSS standards in mind - that technology piece I think has been a pretty big one. It's allowed us to incorporate several kinds of new key activities and stuff that have really made the content that we're teaching in biology more relevant to our students' lives...So it really gets at, at part of the things that our new NGSS standards are going for. So it's really hand-in-hand; these things that we're finding with our technology are really kind of hand-in-hand with that critical analysis piece that we're trying to build in.

This teacher finds the laptops help to facilitate the critical analysis component of the NGSS.

Teacher one also stressed the purposeful use of the laptops for instruction:

Not just for the sake of technology...But when is it going to provide a good learning experience for the kids, or a good data collection experience in the lab. So it's going to add to what we're trying to do.

Teacher one understands the purposeful use of the laptops as tools to accomplish objectives, rather than simply using them because they are available.

Teacher one went on to emphasize how having the laptops facilitated the modeling approach to instruction, and how important that approach has proven to be, “...we decided we needed the computers because the modeling curriculum that we started last school year has data collection with several of its labs.” Following our interview together, this teacher sent an email to me supporting the modeling approach. The teacher stated, "For 23 years. Modeling Instruction has helped teachers attain knowledge and skills needed to benefit their students.” This teacher finds the laptops vital to successful delivery of the modeling approach to 
instruction. Because the laptops are integral to the way this teacher delivers curriculum, they are used regularly in teacher one's chemistry classroom. These findings show the science teachers' curricular goals are being supported by technology to improve overall instruction.

Other teachers reported the laptops have improved their instruction. According to teacher three:

Having the devices right in the back of the room has been, phenomenal... when we say go to course sites, they immediately get up and go to the back to the room and get their computer. And they can get started... They have been valuable to allow students to explore like actual scientists do and help achieve our goal of educating students to be, "scientifically literate."

During the classroom observations I saw the participating teachers using the laptops for a variety of purposes, including having students use them for scientific simulations, showing video clips illustrating everyday applications of scientific concepts, providing access to information, data entry and analysis, and graphing of data. Teacher seven said:

It's changed the way we collect data in some of our labs. It's changed the frequency of the numbers of times that we used the Internet to access information. It's changed the ways that, I guess it's increased the number of ways that students are able to view or access or receive information. I mean, it's added that, that element of interactive graphic animations and things like interactive software that they're able to manipulate and kind of get feedback from.

This teacher realizes the availability of the laptops results in increased access to informational resources, enriching the overall learning experience in the classroom in myriad ways.

Having this access to laptops has implications for what teachers need to teach. Teacher one has changed to putting an emphasis on how students use information rather than memorizing it:

So part of my change has been you know, you can look that up, and you don't even have to have paper anymore to look it up. So I'm not worried about you memorizing that anymore. You have to know what to do with it once you look it up, and that's what the important part is. 
Teacher five has also experienced an improvement to planning for instruction as a result of having the additional laptops:

I can easily find something interesting the night before 'cause I get all these updates for science in the news, and I find something interesting that applies to our class, so I'll pull together a few links, check them out, make sure that they're appropriate for the kids, put them together into a short web quest, post it on Angel or Blackboard, and they can sit down and, and start doing it immediately. It means that I can translate something that comes out on the news services last night and have it in the class next day...timely delivery, real time delivery, you know, science in the news becomes science in the classroom within eight hours turnover time, you know, eight hours because I stay up way too late and look at stuff on the iPad. I say, "I'm gonna use that tomorrow!"

Teacher five’s sharing how, “science in the news becomes science in the classroom” illustrates

how the laptops as tools can make learning more relevant to students' daily lives. Teacher two also shared how the laptops have helped make science more relevant to students' lives:

I think that relevancy piece is definitely there on being able to bring that technology in...one of the examples that was just so - I really thought was really powerful this year was we did a couple of different things. One was researching chromosomal disorders and having the students make some presentations on those. It got at so many different I think levels for the students, but that - technology piece of having those laptops available was able to make that happen. You know we didn't have to schedule time downstairs and do all of that, you know, and again it just really made it relevant to their lives.

While the additional laptops have improved access to information, planning, and relevancy of instruction, the teachers also reported how the laptops have enabled additional instructional benefits. Teacher four summed it up saying, "So it’s just given a lot more opportunities and a lot more tools to do the job.” Teacher six shared how the mobility of the laptops has been beneficial to instruction:

By having mobile laptops, now I can pick up and we can go outside if, you know we want to do a running activity with a motion detector. So for a science class that mobility is very helpful. And so it allows me to engage more in the environment. 
Teacher six went on to explain how this mobility is more closely related to how scientists work.

This teacher also echoed the relevancy factor shared by other teachers, as well as how having the laptops has enabled the teacher to illustrate complex concepts:

The technology just makes it easier on a lot of things to allow kids to see models or to access resources, to do independent projects and stuff like that. Because

all learners learn at different rates, I do think that's where the power of the technology can come in. It's not gonna replace the teacher...I can teach without the technology but to me it's a godsend to have it - Because it just allows me to connect more... So I think it helps them to visualize how the theory of physics at least is being used out there into the real world. So I think it's a great way for me to do that. It also is another way to get extra practice out for kids.

According to this teacher, the additional laptops have provided tools to enhance instruction by providing opportunities to connect concepts for students, and to illustrate complex ones, making them relevant to students' lives. These same points were echoed by all of the participating teachers in relation to how the laptops in their classrooms have improved their instructional delivery. The science department chair summed up the effect the additional laptops have had towards improving overall instruction and the implementation of the NGSS:

We've been teaching a lot of content in science, very shallow. And they want us to decrease the amount of content and increase the depth. And what they mean by that mainly as I see it is they want kids doing more experiments. They want them analyzing data. They want them making, like we're trying to do with this global warming for tomorrow, make a claim but provide the evidence behind it and convince me, communicate to me, to the class, that you're right.

Laptops eliminate the need to schedule access to computers. The teachers work in a large high school with over 1600 students. They described previous challenges related to plan for using computers because they have multiple sections of the same class. Being able to schedule access to computers for each of their class sections was often not possible. According to teacher two:

You know, we've got the computer labs here. We've got three main computer labs, a smaller lab downstairs with the media center, but booking time in those has always been 
difficult 'cause we've got so many other teachers and so many other students, so that part has been difficult and the laptops have just been wonderful for that....It would've been very difficult if we would've tried to schedule all the different biology classes into the computer labs on the same days. That would've presented a huge barrier.

And teacher three explained how eliminating the scheduling factor has facilitated timely use of technology:

And in the past it was very tough to say, you know I'm going to be on gas laws. I have a really great gas law activity on the, ah, PHET website. And I want them to do it. But I can't do it four days from now and that's the only day the computer's open. Well, we'll just not do it this year.

Teacher five also eluded to the scheduling factor and its effect on instruction:

Well it's really nice because before we had to have a real concerted effort to plan ahead, make sure we had a reservation, you know, vet any sources well in advance, make sure that they were gonna load, make sure they were gonna work... It doesn't take a week lead time to reserve the cart, bring it to the class, make sure all of the connections are working, all of those.

And Teacher one explained:

We've always done some simulations, but we had to go to the computer lab, and it was difficult sometimes to go to the computer lab, so it's nice to have them in your room, and if you want to do something that maybe only takes thirty minutes on the computers, it's easy to do. You feel guilty for taking up a lab for the whole period when you're only going to do a short activity.

Having the additional laptops available in the science classrooms has eliminated scheduling challenges related to accessing computers. It has eliminated the need for figuring out how to plan for each of their class sections to use technology, and to allow for the extra lead time it required to reserve time in a computer lab within a large high school. The teachers can now more readily plan and provide activities using the laptops without advanced scheduling.

Laptops facilitate inquiry based and student centered instruction. The additional laptops have provided the tools to enable the teachers provide inquiry driven instruction. Teacher five explained: 
In science we want the investigation to be immediate, to be able to evaluate sources and be able to, you know, be student inquiry driven rather than just canned, and so it really enables more of that spontaneity and immediacy of feedback.

Teacher one discussed the positive effects the laptops have had on instruction because the laptops have:

Helped it become more student centered, more constructivist. More inquiry based. And just I think giving kids ways to learn things in a modality that they may be more accustomed to, because they're such technology babies. They're almost born with a device and a screen.

For teacher one the laptops have provided tools for meeting today's students where they are as, “technology babies” who are, “almost born with a device and a screen”, acknowledging the importance of considering the students' perspectives and for making instruction more student centered. Teacher two also shared the importance of the student centered approach:

Yeah, and being able to research it again on their own rather than just us having to tell them "Okay, these are some of the things that could happen." So again more of that student centered approach rather than "Okay, here's the information."

Allowing students to explore information rather than having the teacher deliver the content by lecturing is facilitated by having the laptops. Teacher one explained:

It has been great having them because we can have students do exploratory pieces that would have been more difficult in the past without the computers...So when it's an exploration kind of thing, I think it can be a good thing because they really don't know what's going on, and they're going to try to figure it out together.

The additional laptops have provided tools to facilitate the delivery of instruction in a more inquiry based manner and has enabled student centered instruction. Both of these goals align closely with the modeling approach adopted by the participating teachers, as well as with the NGSS adopted by the school district as their official science curriculum (Personal Communication, M. Baker, June 3, 2014). The laptops have provided the tools to help achieve these instructional goals. 
Effective instruction is facilitated by print and electronic tools. All of the participating teachers mentioned the fact they need both print and electronic tools. Several shared concerns about not having at least some textbooks for delivering certain content, despite having the laptops and access to the activities and tools they provide. As teacher three shared:

There's some value in print -- to actually have it in front of you for the kids really who struggle too sometimes, there's some value there. And so I think we should always have some available if a kid wants to check them out... So honestly, for us it was a no-brainer. I mean we get computers. Absolutely. We'll go that route...I think the kids have high motivation using the technology as opposed to the standard materials. I don't - what I've learned is don't use it exclusively.

Teacher one also explained the dilemma of not having a textbook because students in Advanced

Placement (AP) courses were also given iPad Minis instead of textbooks:

So I'm torn between if I - do I get a paper textbook or do I just - I mean, I don't even know if they're going to let me get a paper textbook at this point because the AP kids have an iPad. But when you're looking at a Science textbook and you've got a graph, and you've got this iPad mini, how do you look at the graph and read the accompanying text or the problem question at the same time on such a small screen whereas when you have a textbook, and you can look at two pages at once.

Teacher five shared a suggestion for this dilemma:

If I could have my wish and what I would really want is the electronic textbooks and the laptops, because then if I had a kid that needed some I could print off the page off of the electronic textbook. I can get a PDF of it. I could print it off if they needed a physical copy to mark up and they actually would have the textbook to read and they'd have the associated resources in those online learning, um, centers that go with almost every textbook, so that's what I would want.

Teacher four brought a textbook to the interview to illustrate how much easier it is to view complex diagrams and scientific charts from a printed resource rather than a laptop or iPad. This teacher had also performed an informal survey on this topic and shared:

So how's it going this year, and so do you guys have iPads or books, and so tell me about it. The secretary said in our - in our main workroom, "Teachers are coming in here and asking me to copy the entire chapter and print it on the copy machine," [laughs] "'cause the kids want a book in their hand in class." Paper, there's always going to be a need for. Pencils? There's always going to be a need for. We can't throw them away. So books - 
so I did bring this 'cause look... - I can see this whole page. I can see the equations that take me to reading the examples and understand them. I can see the diagram. I can see everything. I can just go boom, boom, boom, boom with my brain.

In order to avoid purchasing expensive AP textbooks to accommodate increased student enrollment in AP courses, iPads were given to students taking AP classes during the second year of the teachers having the additional laptops. This decision was made by the school district level administration without input from the AP teachers. Due to these additional tools becoming available, there was an increased focus on what type of devices are used. As I noted in my research journal after conducting several of the teacher interviews, it seemed to me some of the teachers were motivated to participate in this research so they could advocate for the laptops instead of iPads. Teacher five said:

The iPads don't work with a lot of the interactive stuff. Our textbooks have the things where you drag the things and right now they're not HTML5 so you can't use them on the iPads. You can use them on the laptops, but it's much more interactive with the students if they can touch it and move it around. So we use the smart board a lot.

However, the science teachers all have the additional laptops in their classrooms, despite the AP science teachers also having access to use iPads. Teacher one summed it up saying:

Well I think a big thing that we were happy about was that we had - we were given the option that we really wanted computers rather than iPads...And so I think that's a big deal. Paying attention to what the experts in that subject area say they need. You know, and you have to of course, know that these people know what they're talking about.

The science teachers reported the laptops as being valuable tools, but they do not think the devices can completely replace print resources for delivery of some content. In some instances, the teachers found having the laptops at a ratio of two students to one laptop is sometimes not enough, and a one to one ratio is needed.

Effective instruction sometimes requires a device for each student. During the interviews some teachers described situations where having one laptop for two students to share 
was not ideal. According to teacher one, "If it's a practice, then each person really needs to have their own... So if they're practicing something, then probably I'd like the one-on-one because then I know that each kid is doing it.” And teacher six shared, "And so I think you get more engagement if it's a one to one versus even a one to two.” However, because the teachers work closely together, some described how they are able to achieve a one to one student to laptop ratio when necessary:

There's been a couple different instances where there's been individual projects, but by having two per class, you know, 12 per classroom, we've been able to say "Oh, can I borrow your set for today?" and been able to work it out like that. (Teacher 2)

And so, like in our classroom they're used most days. Somebody's using them. And it's not, you know all 12 come out. There are some days we want every kid to have them. And we go over and get the ones out of the anatomy room if they're not using them today and we have 24. (Teacher 3)

The teachers have found ways to make sure they have the tools for effective instruction, whether it involves having a combination of both print resources and laptops, or whether they need additional laptops for students to use for activities when sharing devices is not ideal. Overall, the teachers would not trade having the laptops in place of printed textbooks, but they did acknowledge the need for both types of tools.

\section{The availability of multiple technology tools enables active learning. During}

observations in the teachers' class rooms I witnessed use of short video clips to engage students, increase understanding, and make the concepts relevant to students. I also saw web resources and simulators being used, Logger Pro software, data analysis tools including Microsoft Excel, interactive games, Google Docs, and Web Quests. Other non-electronic tools such as flash cards and whiteboards were also being used. I often observed students using ear bud headphones. During the observation in teacher five's classroom, students utilized multiple laptop station activities the teacher created to replace what she said had been a, "boring video". (Personal 
Communication, P. Clancy, 4/29/14). During interviews teachers mentioned also using the Bozeman Science web site and Khan Academy. Multiple tools are being used by the participating science teachers. This fact was echoed during some of their interviews. Teacher three shared:

So we've got the smart boards. We have the iPads. We have the computers. We have access to getting kids to see, you know a media-rich environment, in an interesting environment...Computer, smart board, data projector, computers that the kids can use. Their cell phones. Um. Probeware....we have microscopes... I'll tell you the district has been phenomenal at providing the resources when we need and we ask for them.

Teacher five, who is also an AP science teacher, explained:

They have iPads, and you have a lot of the affluent kids have smart phones. Some kids don't, and so if you're using things like Socrative where they can do the online quizzes or the Edupuzzle or those kinds of things, having the Netbooks means every kid has access to something, and so most of these tools that are available online [clears throat] like Edupuzzle or all of these, work across all these devices.

Teacher five introduced the idea of allowing multiple devices in the classroom in order to provide access to additional tools. Teacher three shared a powerful learning moment when the teacher realized some students are better able to use cell phones to which they have become very accustomed. The teacher described the experience:

You know what? I actually had a kid in my remedial class who Googled on the computer last year. And most of them are special ed students who, who are a little bit, you know, maybe reading at a lower level and so they have a hard time picking out stuff. But she was having a hard time Googling. I said, you know can you Google on your cell phone? Cause she's always on her phone. Yeah. Why don't you just Google the Keystone oil pipeline on there and get your information from that? "Can I?" Yeah. She's a, there it is. How about this? Will that work? - And then she started using it. It's like, okay, well, we, we'll roll with that technology.

Technology tools encompass a wide array of applications and types of devices. The teachers reported using the laptops along with other types of devices such as probeware tools for collecting and analyzing data. Some have allowed their students to use the device most 
comfortable for them where they can be more successful. During the classroom observations, it was evident a wide variety of tools provided opportunities for more active learning opportunities within the science classrooms.

However, during the classroom observations, some barriers to using the laptops were observed, and some were reinforced by the teachers during the interviews. Barrier themes within the tools dimension include (1) long log in times, or difficulty logging into the district computer network, (2) software and device compatibility issues, and (3) students who do not have access to the Internet or devices from home. In particular, the barrier of long log in times and difficulties logging in to the computer network were shared by all of the teachers during their interviews and were observed during each of the fourteen classroom observations.

Teacher five explained the login challenges:

There is a problem with how long it takes to get the machines up and going. With those I often will tell them we're gonna use the Netbooks, so get one and get it logged on now because in ten minutes we're gonna use it. So we've gotten around it. Generic logins we use for the general classes a lot, um, and then we just build in a little time for them to get logged on.

And teacher one said, "So the login issue is a big one because sometimes it could spin for a long time. Now granted we figured out okay, if it’s spinning for a long time, grab another one.”

While these two teachers have found ways around the long log in times, sometimes other teachers may not. According to teacher two:

For some reason it's always the struggling students that they get the computer that's not logging in and they've tried four times, and they're still having problems, and you've got the other half of the class, which has already finished with the assignment. So yeah, there's been several that we've just had to kind of scrap and say , "Okay, I'm not doing that for my other classes.”

Teacher three builds in the additional time needed to log in the laptops:

Sometimes a barrier is you gotta remember that if you're gonna use the laptops sometimes I gotta remember to tell the kids ten minutes beforehand... And, and that sometimes I 
forget and then we gotta - cause the kids sit, it's talk time cause they're waiting for their computer to load.

Teacher six reported seeing the situation improve during the time since they have had the additional laptops, “And some computers would never log in. Some would. And, you know we, you know hopefully we've gotten that sort of minimized now.” And teacher six continued:

We're always pushing on the IT department because, you know we expect things now and, you know we don't like it when the network goes down. Um, which it really hasn't this year, knocking on wood. But, you know, we look at it is, in, in terms of my perspective, we're, we're looking at it as, well, you expect us to use these tools and, and, you know things are always gonna happen. But I, I think the environment's gotten better.

The long log in times have not proven to be an insurmountable barrier for the participating teachers, but as teacher six shared, it is important the technology infrastructure be reliable when teachers' instructional delivery methods depend upon it. During one classroom observation, the entire computer network went down for a period of five minutes. Teacher seven had students performing a web quest at the time. When the network became unresponsive, students calmly let the teacher know. The teacher in turn remained calm, first checking a couple of students' laptops, and then inquiring with a teacher next door. Once the teacher confirmed the network was down, teacher seven directed the students to a reading activity. Within a couple of minutes, the network was available, and the students went right back to work on the web quest. When I asked the teacher about the experience after class, teacher seven said you learn to always have an alternative plan when you are using technology (Personal Communication, G. Gray, May 27, 2014).

Students who do not have access to the Internet or devices at home. Teachers reported a second barrier of not consistently being able to assign work outside the classroom because some students would be unable to complete the work without having access to the Internet, or to 
a computing device. Teacher two explained, “They don't have Internet access at home.

Whatever it is, there's those barriers that it's not gonna be a legitimate type of assignment to be able to send them home with.” Teacher five shared the need to provide access to the school outside of normal class hours to help those students with limited or no access:

I don't want those kids not to be able to rise to the same level as the others, because at home they don't have access to, uh, the Internet, and that is a big issue, and we all act like it isn't, but it is a big issue and we are hurting our kids by not having open hours in the evening in the schools.

And teacher six explained even though students may not have access to a computer at home, having it at school at least provides the experience of using laptops instructionally for students:

I could probably teach my classes without the aid of a textbook. So I would have probably have taken the laptops. Because I, I feel that that would give kids that don't have technology at home, which is now becoming smaller, but there are still those. At least some advantage of seeing some of the things and playing with some of the things. Whereas, you know there again if it was just me and I said, okay, well, here's this nice little app that we found out here on Google and, and I'm now showing it to you and the, the link is up there on Angel and you can play with it on your own leisure. Well, some of those kids aren't gonna be able to do it.

While a lack of access to the Internet or devices at home does not provide a barrier to teachers using the laptops instructionally, it does present them with special considerations when it comes to their being able to assign work requiring a computer outside of the classroom. The findings show some of the participating teachers maintain a level of sensitivity to this particular challenge.

Software and device compatibility challenges. The third barrier evident within the tools dimension relates to the teachers reporting occasional challenges with software versions, as described by teacher four:

So typically, everything works real well. Software works well. Sometimes there's compatibility issues like Java updates a new version and all of a sudden, your software is not doing what it's supposed to. Most people don't know how to mess with it. Most people get frustrated and I just say, "Okay, I have to figure it out," boom, and fix it. 
Teacher four indicated being comfortable figuring out the incompatibility issues and is able to fix them for others. Teacher one offered advice for using the laptops despite knowing occasional issues may arise:

Practice before you give it to the kids. Make sure it's all going to work. Don't assume. Don't assume anything is going to work the way you think it's going to work. Especially with different platforms in terms of you know, the iPads not - you have to have Puffin for the Flash, or the Java, or you know.

And teacher six referred to the challenge of software changing, especially after teachers become accustomed to using it:

And I think when things software wise start to normalize, because right now there are teachers out there like, you know I have always been on the bandwagon of, okay, I'm using Blackboard. Oh, it's too clunky. It does this. Hey, we moved to Angel and hey why don't you - oh, it's too this. Well now it's sort of that, well, they're gonna change it in three years. So why should I even bother? And I'm sitting there going, yeah, don't say that. But yes, it is, you know. So there, there is that piece where there has been so much change going on.

The challenges shared by the teachers relate to the constant change occurring with technology. The compatibility between software versions and device capabilities do not present barriers the teachers are unable to overcome. However, the constant change requires their awareness and attention in order to be able to effectively use the laptops with students in their classrooms.

Overall within the tools dimension, the teachers reported finding the laptops to be valuable tools for improving their overall teaching, for providing readily available access to powerful computing devices, and for allowing them to provide more inquiry driven, student centered instruction. All of these themes were viewed by the teachers as facilitative factors within the tools dimension. From what the teachers indicated, the facilitative factors outweigh the barriers they experience with the laptops. Issues of slow connectivity, students not having access to 
devices or the Internet outside of school, or software compatibility issues related to constantly changing technologies do not present insurmountable barriers for the participating teachers.

\section{The Knowledge and Skills Dimension}

The knowledge and skills dimension includes what is known as well as the content of what is needed to be mastered. Within this dimension, three facilitative themes emerged. No barriers related to teacher knowledge and skills were shared during the teacher interviews or witnessed during classroom observations. Overall, the teachers' statements indicated they feel they have the knowledge and skills to use the additional laptops instructionally. During the classroom observations I also witnessed the participating teachers' skill with using the laptops instructionally. I observed the teachers advising students about how to work around the login challenges which arose. I also watched them successfully handling and managing the logistics related to laptop power length and proper storage of the devices. Sometimes teachers had the laptops setup and ready for class use, and other times students accessed the devices from the storage carts, depending on how and when they were being used during the class period.

Their knowledge and training in relation to the modeling approach to instruction was mentioned frequently when the teachers were asked to discuss facilitating or barrier factors related to their knowledge and skills and the use of the laptops with students. Within the findings, the three themes within this dimension include (1) some teachers prefer learning about technology independently while others learn from their colleagues, (2) knowledge about the modeling approach facilitates using laptops instructionally, and (3) teacher knowledge is sufficient, but they are open to learning more.

Teacher knowledge and skills vary according to their personal interests and experiences. 
Some described being very adventurous and interested in learning about technology in general, and others prefer to wait and learn what they need to know about technology from their colleagues. Teacher four shared:

Well, my knowledge and skill is I'm a scientist nerd, so technology doesn't faze me. I tear it apart, fix it, break it, fix it again, set it up, have it do things nobody else thought to have it do, so it's not any hindrance at all. It takes time to mess with it to make it work, so that the user-learner, in this case students, get something out of it and they aren't bogged down in just the technology... I spend way more time than I should. [Laughs] I work for free a lot.

And teacher six echoed the same type of interest level:

I mean I've always liked technology so, I mean back when I was a kid I, you know, took computer programming as a sophomore and had an Apple IIe...So I, I've been very spoiled and I've kept up with the technology to a certain degree. I mean my programming days are probably way gone. And I enjoy that, and so I think it does help...I see possibilities and I don't like to be limited.

Teacher five described how technology has played a role in the teacher's prior career, and her interest in technology has continued to the point the teacher furthers her personal knowledge using Twitter and blogs (Personal Communication, P. Carney, May 7, 2014). Teacher five explained:

I worked for a bio tech company and so they were, you know, using email, uand the Internet before it really became part of the general lexicon. So I was probably in on the ground floor with a lot of that, some of the earlier, earliest World Wide Web in town were done in the database project, which I was working in at the time. So it's always been just, you know, it's a tool just like a pencil and it's there and I'm gonna learn how to use it as much as I can....but as far as it's not something I'm afraid of... I'm always anxious to learn, and so I have taken probably everything you guys have ever offered through the PD (professional development) here. Um, I've also just finished, I finished a big MOOC (Massive Open Online Course), um, through MIT... So I'm pretty comfortable and I'm really not too afraid to play around and try things and see.

Teacher one also indicated a comfort level with computers, and a high enough level of interest to work with them during personal time:

Because we bring the probes home and attach them to the computer at home, and mess with them and collect data and figure out how it's going to work, or do it on the weekends. 
So not being afraid of computers and not worrying about I'm going to break it, or I'm you know, going to mess something up. And just you know, taking the time to fool with it before you do it with the kids obviously is important. And so my knowledge base helps a lot.

Teacher three indicated his knowledge was furthered and impacted by attending a national conference and being motivated by a keynote speaker. Teacher three described the experience with enthusiasm during the interview:

A year ago, thanks to the district who paid for all the department chairs to go to San Antonio for the, ah, ah, National Science Teachers Convention. I got to watch a gentleman named Paul Anderson who was a keynote speaker... he has a YouTube channel that he calls Bozeman Science. Come to find out, no, he's a phenomenal AP and regular biology teacher in a little, in a community that has high poverty. He's in Bozeman, Montana. And he has not flipped his class but had a blended class, and I'd never heard of that. And so I came back here and shared that with our science department. Because what he said was it's not about you making the videos on your iPads or your Macintoshes. And, and just making them watch it at home. It's not about that. It's about freeing up your time so that you're not always at the front of the room talking to a group and not knowing if the group is paying attention or not.

Teacher three has taken this knowledge and is applying it to biology instruction, using the additional laptops to facilitate this blended flipped approach to instruction (Personal Communication, D. Martin, May 20, 2014).

Teacher three also shared some advice about those teachers whose interest and knowledge are not the same when it comes to instructional technology use, advising, "I would give the technology tools first to the teachers who are anxious to use them... And so those teachers can then become your professional development.” Teacher seven, when explaining a willingness to use the laptops, but not having as much time or willingness as other teachers to spend personal time developing the knowledge, shared an appreciation for colleagues being willing to share knowledge, "And different teachers in our group spend time experimenting and surfing around and trying to find different apps and different, you know, animations and different video clips 
and they share that.” Teacher two concurred sharing:

So, being able to build those pieces of technology in again both for delivering information, that content, and then assessing it through technology... you know, having other teachers that have used it and applied that type of stuff I think, is probably more of that ideal... that teacher delivered piece is just, is huge.

The teachers' varying levels of interest and some being willing to further their knowledge about technology through experimentation and investigation has enabled all of the participating teachers to benefit and develop sufficient knowledge regarding the instructional use of the additional laptops.

\section{Knowledge about the modeling approach facilitates using the laptops instructionally.}

When teachers were discussing the knowledge and skills they have about using the laptops, the modeling instructional approach was frequently mentioned as being a facilitative factor. Teacher one explained:

So all the teachers, we've all had at least three weeks of training. Some of us have had up to five weeks of training in the modeling curriculum. And so through that we had some training with the Vernier software Logger Pro. That's the software used for data collection and graphing. So we had some training in our summer training program through modeling. So that helped because if one of us didn't remember how to do something, maybe another one did, and that was good.

The knowledge acquired during modeling training involved software the teachers use with the laptops. A majority of the participating teachers adopted the modeling approach to instruction just prior to the laptop implementation. During many of the classroom observations, I observed the students using the Logger Pro software to enter and analyze scientific data. This analysis aspect is facilitated by the laptops, and integral to the modeling method. As teacher seven explained:

We adopted the modeling curriculum in chemistry last year, and we used it last year, and then we went to a seminar again this summer, and we used it again this year, you know, obviously with modifications and things we learned the first year... I think, what we try to do with it is to have students develop their understanding based on evidence, and then to, 
rather than just give them the answers, and have them memorize, and, regurgitate, it's, it's show them evidence and then... have them try to make sense out of it, of an explanation that fits their observations... So I think it works well... my feeling is, is that students have a better understanding of what they're learning rather than just being able to plug numbers into an equation and get a correct answer. They actually understand what the numbers represent.

And teacher four described how the modeling approach is being used by chemistry and physics

teachers. Teacher four was a leader in bringing this approach to their science department:

"We need to start doing something like modeling", I said. And, well, chemistry hadn't come around yet, either, -- countrywide--, so the modeling people started with physics, then they started moving to chemistry, and so finally there's some training that could happen in modeling. So one teacher went one summer, one went another summer, and I'm sitting there going, "It's time to change."...So last year, chemistry went modeling at our school... as an instructional method.

Teacher one, when speaking about using the laptops within the modeling curriculum, shared,

“I’ve actually used it - used them more this year than I did the first year I think partly because

it's my second year teaching the curriculum.” This second year of having the laptops has

complemented the additional knowledge and skills the teachers have about the modeling

curriculum and its instructional approach.

Teacher knowledge is sufficient but they are open to additional learning. The teachers

indicated being comfortable with the knowledge and skills they have for using the laptops. Their

background knowledge provides them with enough skill to use the laptops with students.

Teacher two explained:

I think most of us are pretty comfortable with the PC-based stuff... the laptops have been really good as far as that transfer of knowledge on our part on how easy are they to use, how much extra effort do I have to put in to come up with some assignment that they can do on there.

Teacher one concurred, "Having had the training ahead of time with the software in the summer was very valuable because then you don’t have to figure everything else - everything out at once.” As did teacher seven stating, "We have obviously more background knowledge than the 
students have going in, so we know more what to expect, and kind of questions to ask that students may not have 'cause they don't have the background knowledge..." Sharing how he has learned to try things first himself teacher seven also explained:

We always try to make sure that we experience - we do the lab that we're gonna ask the students to do ourselves first, so that we have that experience, that we know, "Oh, well, in order to get it to work properly"... so we, we have that experiential knowledge going in.

And teacher five advises:

So you just got to have a plan B because the Internet might go out and then you don't have anything, so you've always got to have some backup, something to pull out your back pocket... So have a low tech backup, but don't be afraid to try new stuff, and let the kids help. The kids can solve problems.

During my observation in teacher seven's classroom when the Internet connectivity was completely lost, I witnessed the teacher putting plan B into practice, having the students smoothly and efficiently switch to a reading activity related to the lesson.

Teacher five has learned developing knowledge in bits and pieces works best, and offers this advice:

You don't have to have the perfect lesson and don't try to make the whole perfect lesson you've scripted out all-together. Just do a little piece of it, you know? Have a little bit of it here, have a little piece of it there. After a while you'll find what works.

And teacher two shared a need for developing additional knowledge:

I would love to be able to incorporate and I need to be able to incorporate ways to assess ways to get information to students, to be able to do interactive things in online webs, labs on the web for those AP students because I just physically can't do that in the classroom as a class within a class type of situation... So, being able to build those pieces of technology in again both for delivering information, that content, and then assessing it through technology.

Teacher six offered advice about increasing teachers’ knowledge using PLT (Professional

Learning Team) time to do so. Teacher six recommended the exchange of knowledge during this time should flow naturally and not be prescribed or mandated. (Personal Communication, P. 
Stone, May 21, 2014). This advice means school leaders who want PLT time used for teachers learning about technology would need to insure there are teachers on each team who can enhance the knowledge exchange within the team as it relates to technology. It also means PLT time potentially could be an optimal time for teachers to increase their knowledge and skill about technology, learning from their peers within the context of lesson planning and evaluation.

\section{The Environment Dimension}

The environment dimension encompasses the educational setting, including the norms, expectations, and interactions comprising the school culture. Findings within this dimension centered on the teachers' PLT time, the school's new block schedule, and an overall collaborative, respectful school culture dedicated to improvement. Four facilitative themes emerged within this dimension, including (1) professional learning team (PLT) time enables teacher collaboration and learning, (2) block scheduling facilitates learning and laptop use, (3) school culture represents sharing, diversity and academic excellence, and (4) professional development (PD) opportunities encourage learning and sharing. No barrier filters where shared by the teachers in regards to the environment dimension.

\section{Professional Learning Team time enables teacher collaboration and learning.}

Teacher three explained teachers have an average of 90 minutes set aside every other day for PLT time. The participating teachers shared only positive comments about this time. According to teacher three, "So that collegiality has just bonded, and made us both better instructors. And I think and really enhanced our use of the technology.” Teacher two elaborated on using PLT time for learning from colleagues about not only the tools, but also about improving instruction:

By learning from other teachers you learn not just that hardware piece if you will, you learn all the other things that go with it, and you learn more background on whatever topic it is. You learn again different ways that you could have it. You learn ways that you can differentiate that instruction for, you know, your students that are doing well. You 
learn so many other things in addition to that one piece. So not only do we come up with, say, you know, a common assessment, but we also benefit in so many other ways...

So, yeah. So if you can reinforce how beneficial those are. [Laughs]

This teacher was advocating for the school district to make sure this time continues. Teacher three explained how valuable PLT time is:

Unless we happened to have lunch shift together, we never had the same planning period. You know we are on different schedules, different activities. And, and we didn't plan together much. So now everything we do is the same. We meet constantly. We are developing formative assessments...I have to say we're pretty protective over that PLT time that we have...it's just been so wonderful being able to have that time. It's just so productive.

Other teachers discussed how some teachers are more interested in learning about technology than others, and the time set aside for professional learning teams allows time for those less willing to learn about technology to learn about it from their colleagues:

And different teachers in our group spend time experimenting and surfing around and trying to find different apps and different, you know, animations and different video clips and they share that (during PLT)... there's, the time element is always, well, oftentimes is the critical factor, you know, I'd like to do that. I'm motivated to do that. I have the equipment to do that, but I don't have the time. (Teacher 7)

Teacher two concurs, "Um, again, you know, having other teachers that have used it and applied that type of stuff I think, um is probably more of that ideal... but I think that, that teacher delivered piece is just, is huge.” Not only is the PLT time valuable for learning together, but when it comes to learning about using technology instructionally, this teacher indicated a preference for learning from colleagues.

In addition to leaning from each other, the teachers described how PLT time also allows them to extend their learning into developing lessons and activities to improve and extend learning during PLT time. According to teacher seven, "We have used PLT time to kind look at animations and see, "Okay, we'll compare them. We'll look at that one and look at this one and 
look at this one. Which one do we wanna use?” Teacher seven shared, "the people that are in the biology PLT will find video clips from YouTube that fit with the lesson...” Teacher five, who is a teacher willing to learn about technology independently shared:

I seek out a lot of PD on this stuff but have very little time to actually develop products and work with things and work with others to develop products. So as you're looking forward to trying to help others incorporate more of this technology into the classroom, what has helped us immensely in science is having these PLT times where we've actually pulled out the stuff and worked on it and come up with things together, giving people those opportunities to do that, not just "Here's a quick workshop how to use this tool."

Teacher six summed up a common theme shared during the interviews with all of the participating teachers, "PLT time has now become very sacred...” And teacher five offered some ideas for extending the use of PLT time for teacher learning:

It might be a matter of going around and doing some things in the PLTs with people who actually do work together who are developing lessons to use in their classrooms and they're a team already...Y Yeah, or maybe the PLT be able to go down and work with the media specialist or somebody, you know, during their PLT and learn how to do something together as a group, so that if you didn't catch something somebody else can catch it and be able to use these tools to their potential...I think giving people that collaborative time to work and develop stuff, not fake stuff but real stuff that they're gonna use, and it needs to be done within a PLT I think so that they can get feedback right away.

When I inquired about taking time to observe colleagues in their classrooms, teacher two offered this assessment of learning from other teachers, “I don't think, we don't, typically we don't have the chance really to get into anybody else's classroom. Now could we? Yes... I think time is better spent again doing that PLT and that curriculum development piece.” This teacher finds the PLT time more valuable than classroom observations of other teachers.

The participating teachers report PLT time as being valuable in multiple ways. The time has provided opportunities for teachers less inclined to use the laptops to get ideas and support from their colleagues who are more comfortable with using them instructionally. PLT time has facilitated teachers learning from their peers. The teachers' comments show evidence of the 
participating science teachers using the time for critical reflection and collaboration about

teaching their lessons. Collaborative planning, and most importantly, a shared sense of responsibility for student success, are also results of the PLT time the teachers have.

Block scheduling facilitates learning and laptop use. For the past two years, this high school schedule has consisted of four block periods per day, including time built in during those periods for advisory time with students. The participating teachers generally found the extended, block periods helpful for collaboration. Teacher two shared:

So by going to block schedule and again so many things dovetail here, but going to block schedule has really allowed us to build it, that collaboration time, and that's been huge. Us in the biology department, we have collaborated in the summers traditionally every year, meaning whether that's once a week or for a week's chunk of time or whatever to be able to share both lessons, activities, planning time, those kind of things but it's, it's really been beneficial as far as what we have been able to do and how we have been able to do it as far as realigning our curriculum with the new NGSS, with designing common assessments, common objectives, putting us more on the same page as far as those activities and by being able to put our heads together on it. It's been, I think, incredibly powerful, and I've seen such huge differences in my classroom on that student achievement piece.

For teacher five, not only is collaboration among teachers important, but also with students. The block schedule allows for advisory time, and one teacher has found the additional laptops helpful for advisory students:

Collaboration as far as helping kids, individual kids, helping their achievement, keeping track of their work, helping them develop study skills, we do a lot of that with our advisories... And I will have to say my kids in my advisory in this room feel like they have landed, you know, in a gold mine because I have the Netbooks here.

Other teachers have found the block schedule allows them to go in to more depth and to include activities requiring more time, especially those with the laptops:

The block I think helps because sometimes in a fifty-minute period it would have been hard to do the intro, do the activity, do the graphing, and then come back in and talk about it and not run out of time. So the block period helps, especially if we have any login problems. (Teacher 1) 
Teacher two elaborated on how the longer periods allow for going into more depth:

The block periods I think have really helped. I personally am a big fan because I think that they really allow the students to go more in depth. They allow students that time to explore on their own, do more of that inquiry piece versus we have such a short amount of time, let me give you that information. So that I think has really helped so we can build in some of these longer assignments and stuff and have that bigger chunk of time to deal with.

While some of the participating teachers found the block periods a facilitating factor to implementing the Next Generation Science Standards (NGSS) standards, one teacher viewed the block schedule as neither helping nor detracting from using the laptops. Teacher five explained the block schedule is a, "neutral piece”, but having the laptops to use has helped with overcoming the challenge presented by the longer class periods. This teacher shared:

We like to shift gears about every 20 minutes, and moving to the laptops is a way to shift gears, and so kind of sets up a different environment, educational environment. So if they had to do this for the one topic then we switch to this and do the other topic and I maybe won't lose them as easily as I would have if I was trying to do that in the second half of a 90-minute block... This has been one of our tools to try to overcome that.

During almost all of the classroom observations, I observed much of what these teachers described. During some chemistry classes for example, the teachers had ample time for introducing the day's objectives, reviewing content, implementing the learning activities, and concluding the learning with follow-up discussion.

Overall, the participating teachers find the high school block schedule to be a facilitating factor when it comes to implementing their curriculum and using the laptops as instructional tools to enhance learning activities for students. The longer block periods allow more time for in-depth exploration and learning activities as well as valuable discussion time. The extended periods also enhance opportunities for teacher collaboration and learning.

School culture represents sharing, diversity and academic excellence. All of the participating teachers described their school culture as being open to diversity. They view their 
school's diversity and emphasis on academic excellence as facilitating factors to their

environment. Teacher five described the school's culture explaining:

I see our schools as an extraordinarily diverse school and we're very enriched by that, and our kids leave here with an understanding of how the world works... we celebrate everybody's differences and contributions and enthusiasms and we recognize that nobody has to be the same but that we all have something to bring to the table.

Teacher three explained how this school year environment and culture is different than in the past because ninth grade students have joined the high school. According to teacher four it has been a, "big change year", and having the PLT time built into this high school schedule for the teachers has been very helpful and helps the new teachers become a part of the school culture (Personal Communication, M. Blake, June 3, 2014). This teacher went on to share about a ninth grade teacher who joined the faculty this year:

She's really happy to be in an open, democratic, PLT. That's truly - truly collaborative and going, you know, that kind of doesn't work that way and, you know, you can tell me that and I can tell you that and everybody accepts each other... That's truly - truly collaborative and going, you know, that kind of doesn't work that way and, you know, you can tell me that and I can tell you that and everybody accepts each other.

Other teachers also described the openness and focus on academic excellence:

I think we've always had a really good culture. Um, I think we value both the academic side of things, the person as a whole as a student, um, as well as valuing the individuality and diversity of people. Um, I think we've always had really high standards, uh, for both, um, you know, behavior stuff and the academics...” (Teacher 2)

And teacher four shared some adjectives about the school culture, "Open, diverse, collegial, um, a little more, uh, academic and better than it used to be ...just really got a culture that's cool... Diverse, accepting, um, it's just really a good place all around for everybody.”

In addition to being open to diversity and having a focus on academic excellence, teachers described a sense of openness towards each other and a shared sense of responsibility for student success. Teacher three, who serves as the department chair, shared: 
And the culture of the building is we're doing this because your kids are my kids. So if your kids are struggling and I've got them in an advisory, let's get this down where I can help them and get them going. And if my kids are in your class, move them around. And so we're, we're sharing kids...they all belong to us.

Teacher one also discussed this sense of openness and sharing:

Everybody's really open to having people in their rooms, so at any time if you want to, you can... And we do that, and so we've always been very open to that sort of thing, so if someone's new teaching a subject, you know, they'll come and sit in. (Teacher 1)

Teacher six talked about the culture across departments within the school:

I think we have good camaraderie between the departments. You know we're, we're still big but yet people interact. We, you know, there's always gonna be people that are gonna have some type of conflict here or there. But I think as a whole it's a good working environment.

Teacher six went on to explain, "I think it's very trusting type of environment and we know that, you know whatever we say or do, yeah, there might be decisions based upon it, but I think we feel safe enough to share those ideas.” The culture of sharing and being open to diversity and academic excellence facilitates teachers' collaboration and learning from each other, supporting the other themes found within the environment dimension.

\section{Professional development (PD) opportunities encourage learning and sharing.}

When I asked the teachers about collaboration across departments within their school, they all stated there was little time for sharing with others outside the science department. However, overall, they find collaboration with teachers in other departments is facilitated by district wide PD opportunities. Teacher five explained, "I'm talking about your district PD stuff that you have. So that's my opportunity there to talk to people across disciplines... we talk back and forth across the room what people are doing...”

Teacher seven recalled incentives for district wide PD and the opportunity to share with other teachers: 
Well, like I said, they have in-services for training teachers how to use technology and they offer in-service credit for it most of the time. There's a - teachers are encouraged to share what they have learned and used. And they, they're given incentives to do that. They're offered, you know, double hours or whatever of inservice credit for doing that.

Teacher seven went on to explain specifically about district wide PD for the science department, and how the support for it has been beneficial:

He (one of the original developers of the Modeling Instruction) came here last summer and was here for three weeks and taught 20-some science teachers from this district and from other districts that came even from other states, and our district paid for that and they paid us to go. So, I mean, the, there's a lot of support and encouragement, I think, from, from our district and from our department to use the technology and to stay up on what's going on, and help improve what we're doing in the classroom.

In terms of implementing the Next Generation Science Standards and the modeling approach to instruction, one teacher shared how allowing for more cross departmental collaboration would be beneficial:

I know as we're trying to incorporate with our new NGSS standards more writing, more analysis of things, it would be really nice to be able to talk to the language arts folks and, you know, we're not, we're not really taught how to teach writing or how to necessarily interpret things in some ways. Or what strategies are they learning in their language arts classes when they read a piece of non-fiction to pull out key ideas? Those types of strategies so that we can be reinforcing those strategies that they're already learning instead of kind of fumbling over here and coming up with our best ideas on ways that they can do that. So that would be a really nice piece to be able to see, that kind of cross-curricular stuff. Also in the math field as we're bringing more math into our biology curriculum, being able to again be reinforcing and building on what they're doing in math.

Other advice from the participating teachers regarding district wide professional development includes the importance of incentives. The department chair recalled being paid for attending modeling training during the summer (Personal Communication, D. Marks, May 20, 2014). And teacher seven summarizes the message, "Um, continue to offer training....and incentives to instructors, to teachers.” 
The teachers reported facilitative factors related to their environment, with their professional learning team (PLT) time being a major contributing factor to facilitating their effective use of the additional laptops in their instruction. Additionally, their school culture consisting of trust and openness to sharing contributes positively to using the laptops to further the school's emphasis on academic excellence. Finally, the teachers appreciate and benefit from the school district's professional development opportunities. The PD allows teachers to collaborate across disciplines and schools, and in some cases, school districts. However, the teachers made it clear the incentives offered to attend district wide professional development are viewed as a necessary facilitating factor of their willingness to participate in those offerings and they would like to have more cross curricular professional development opportunities.

\section{The Teacher as Learner}

At the center of the ecology of resources framework and sociocultural learning theory guiding this study is the teacher, situated as the learner. The teacher can exert a two way influence on the four dimensions comprising their context, while within each context the filters can facilitate or inhibit teacher learning. A large part of the interviews with the participating teachers focused on the teachers as adult learners, including what motivates them to learn and how they learn best. Findings from the interviews reflect much of what the adult learning literature concludes; as adult learners, teachers are motivated to learn because they want to do their jobs better, and the way they learn best varies by individual preferences.

Teachers want to improve and challenge themselves. The participating teachers consistently shared a desire to improve their work and to keep things challenging and interesting 
at the same time. Teacher two said, “...most of us aren’t ever satisfied with leaving things the way they are and we're constantly changing and improving stuff.” Teacher seven echoed the same motivation:

I'm always looking for a better way to do something. If it's - I don't think - I've been teaching 19 years now and I don't know if I've ever done it twice... have an open eye and an open ear of what's going on out there.

And teacher four said, "Uh, you change with life. You change to get better." Teacher four went on to explain how a song lyric best captures his thinking about change and improvement:

Melissa Etheridge..."Nothing stays the same except change."... Nothing stays the same. But change keeps changing. That stays the same, so I just kind of was brought up that way by my parents. You know, accept life, it changes, grow, learn, learn from your mistakes, learn from your good things, whatever.

Teacher three said simply, "So what motivates me to change? I want to do a better job.” Teacher one thinks if teachers don’t think forward, “We wouldn’t be where we are.” And teacher six said, “I don’t want to consider myself to be a stagnant teacher. And so I am always trying to improve things from the year before.” Teacher three summed up what is common in adult learning literature about the concept of relevance, and its importance as a motivating factor for adults, "I learn when I'm ready to learn. When I'm needing to know something, I hear something new that ties into what I already know but extends it, I learn. And I, I'm interested.” In terms of making adult learning relevant for teachers, many of the participating teachers indicated a primary factor to their motivation for learning is to improve learning experiences for their students because they care about them.

Teachers want to improve instruction for their students. The participating teachers indicated a strong motivation to make their instruction better for students by making it interesting, fun, and more student-centered because they care about their learning experiences. Teacher one said, “...caring about students, wanting them to succeed...That's where my 
personal passion is...feeling like you made a difference to somebody's life.” And teacher three stated, "I try to look at everything through the lens of what’s the best thing I can do for my students.” Teacher seven reflected, "I guess that motivates me, just wanting to make, just wanting it to be better for, for the kids. I want 'em to enjoy it and get something out of it.” And teacher one touched on making learning interesting and fun stating, "I figure if I'm more excited about it, the kids are going to be more excited about it.” Teacher one went on to explain how a teacher's engagement and excitement about the learning activities affects how students respond because they can sense it from the teacher. This teacher shared how a colleague who had been having a difficult time in her personal life during one school year noticed it affected her students' performance for the school year (Personal Communication, J. Murphy, April 4, 2014). Teacher five echoed the same sentiment, "I love science...So I want them to have fun with it...My goal is to get them to do and think and be excited about science, and understand it.”

Teacher five went on to explain the importance of student centered, inquiry based instruction, “....in science we want the investigation to be immediate, to be able to evaluate sources and be able to be, um, you know, student inquiry driven rather than just canned.” And sharing how taking the time to learn about modeling instruction, teacher one explained the motivation, "We were looking for something that was more student centered, less teacher directed. Less direct instruction. And that would hopefully help students gain a deeper understanding." And teacher four summed up why continuous learning and improvement is important as a teacher:

Students should feel successful and be learning what you want them to learn. So whatever can happen to make that happen without coddling, holding hands, or whatever, 'cause they do have to be independent learners as they will in real life or as adults, whatever. 
Teacher four touched on students becoming adults who will hopefully one day be independent learners. Teacher three related the same idea, "We're just like the kids." Teachers are grown up kids who reflect different learning styles and preferences.

The findings about how the participating teachers learn best reflect a variety of individual learning preferences, including learning by doing and learning through collaboration and observation of colleagues.

Learning by doing. Consistent with adult learning literature, many of the participating teachers expressed a preference for active learning. They prefer to learn through practice. Teacher seven's response to the question, "How do you learn best” reflects the importance of active learning. Teacher seven, who learns best by doing, said:

Um, by doing it. You know, it's always been the way I've, I've learned best... as far as really something, uh, becoming, oh, I don't know, so, something that I feel I know it very well, I have to do it.

Teacher two also learns best by actively doing and practicing, "By doing. [Laughs] Definitely, and I'm a very, I'm a very big, um, visual type person as far as I've got to, I've got to see it, I've got to write it.” And teacher three said, "I learn best by somebody showing me and giving me some time to go out and explore.”

When talking about learning and exploring new technology, teacher five shared, “I’m really not too afraid to play around and try things and see.” And teacher four, another teacher who indicated an interest in experimenting with technology independently, shared, "I go try it after reading it, read some more, interact with whatever I'm trying to learn...I read and actually practice.” Teacher one said simply watching someone else is not enough:

I'm the kind of person that likes to get in there and do it, and try it. And if I'm just watching somebody else do it, I'm not going to learn as well as if I'm doing it myself. 
And teacher six described optimal learning as, "Hands on.” This teacher went on to share, "I believe you do learn best by doing things. And if you fail at it, that's fine...I do believe the active part of learning takes place when you are directly involved.”

The significance of active learning opportunities for teachers cannot be overlooked. Providing relevant learning opportunities allowing teachers to try experiment is what these science teachers value. These findings are consistent especially with findings from Fullan (2008) and Schon (1983), where reflection and action are vital components for learning and change to occur.

Learning through collaboration and observation. Four of the seven participating teachers indicated a preference for learning from modeling others and through collaboration. According to teacher one, "I think it helps my learning when I see something graphically, see animation, see models, and can simulate things... Interacting with people is big for me” And teacher two discussed learning in a manner reflective of how the teachers have been learning from each other during their PLT time:

See, it takes that one person that'll, a couple people that'll really start playing with it. And then they go, hey, if they're willing to share it, you know this, check out what I did and I think this would be a great thing to do. If you have a receptive learning team...”

Teacher five continued to emphasize the importance of learning from other teachers who are teaching the same content:

My best education was the first year I was hired to teach... I was learning from teachers, and ever since then I've sought out teachers. I don't like to go to a room with some expert who wrote a book, you know, some expert from out of town with slides. I want to learn from people who've been in the classroom recently, know what they're talking about, deal with real teenagers.

And teacher seven offered advice about how to encourage teachers to want to learn about using 
the additional laptops. Teacher seven's advice reinforces teachers learning from observing their colleagues and through observing them, "I think that once they saw how, how slick it is, and how easy it is to use, I think it might get, you might get more people to buy in.”

When it comes to teachers learning and using additional technology tools with students, the participating teachers indicated a strong motivation towards wanting to improve their work, which means improving instruction for their students. The teachers' preferred learning styles vary by the individual.

\section{Summary}

Overall, the findings of the study suggest the participating teachers have found the additional laptops in their classrooms to be useful tools for implementing the Next Generation Science Standards (NGSS) and for delivering their instruction in accordance with the modeling approach they have adopted especially in the chemistry and physics disciplines. The teachers reported only facilitative factors related to the people with whom they work. Support from colleagues and technology staff members was widely praised by the teachers. The learning community within the school, including students and teachers, provides as much support as the technology staff at the building or district levels. Within the tools dimension, the teachers indicated a need for additional, multiple varieties of tools, including both print and electronic resources. While the laptops are viewed as effective tools for delivering their curriculum, according to the teachers, they cannot fully replace printed textbooks. According to the teachers, we should be adding tools, not replacing tools. The teachers also report observing a need for students to select the technology device of their choice, depending on what they are most comfortable with. However, when it comes to more specialized learning activities required by 
the science area such as using probeware and special software programs, the device does matter because it must be compatible with the peripheral tools. The subject area experts should advise on the tools, according to the teachers.

Some barriers of a logistical nature were reported by the teachers because the laptops often have login or connectivity issues. However, those barriers do not affect the teachers' willingness to use the laptops instructionally because they are seen as effective tools for engaging students in the inquiry based, student centered approach emphasized within the NGSS and the modeling approach to instruction. The barrier of some students not having access to the Internet and devices outside of school impacts the type of activities teachers can assign for homework. Teachers reported having sufficient knowledge about using the laptops, primarily because they learned to use them as part of learning about the modeling approach.

The teachers did not need explicitly stated expectations from their leaders to use the laptops because the leaders' stated expectation is to improve instruction by engaging students in relevant learning activities. The laptops are simply tools to accomplish this goal. Additionally, leadership support by providing those tools and incentives for teacher learning was reported by the teachers as being the more important factor. Overall, the teachers describe their school's environment as a collaborative one known for its focus on academic excellence as well as a prevalent respect for diversity of thought and culture.

The teachers' responses clearly indicated a preference for learning from those with whom they work. Teachers with a natural interest regarding technology reported being willing to learn about it from outside resources such as district staff, online tools, and conference attendance. All of the teachers indicated a preference for learning from their peers. They reported being 
motivated to learn by a desire to improve instruction, making it exciting and relevant for both themselves and their students. As adult learners, the teachers' learning preferences vary by individual, just as they do for students, including active learning as a vital component.

The finding which was reflected in teacher responses repeatedly, spanning across multiple dimensions within this study's framework, is the importance of professional learning teams (PLTs), also known as professional learning communities (PLCs). In analyzing the findings, the PLTs serve multifunctional purposes for the teachers. The teachers report their PLT time as very valuable for learning about the laptops, planning for and improving use of the laptops for learning activities, collaboration and promoting a shared sense of responsibility for student success, and finally, for problem solving, including sharing strategies for overcoming the logistical barriers reported with using the laptops instructionally. Teachers who are willing to learn and experiment with using technology with students reported sharing their knowledge with their peers during PLT time. Likewise, the teachers who do not want to spend extra time learning about technology reported being willing to learn about it during their PLT time within the context of planning for and improving instruction. Overall, the participating teachers reported a willingness to change and improve when they see a relevant, compelling reason reinforced by their curriculum and instructional approach, and when their peers endorse technology tools which align with their instructional delivery.

In the following chapter, I will discuss these findings and their relationship to the relevant literature, as well as conclusions about this overall study and its implications for future research and practice. 


\section{Chapter 5: Discussion, Conclusions and Implications}

The purpose of this single case study was to explore and understand high school science teachers' views regarding the use of laptops they received for their classrooms in place of printed textbooks. This study also explored teacher perspectives about themselves as adult learners, and how they learn best. In essence, it investigated high school science teachers’ personal and situational influences affecting their instructional use of laptops.

Using the ecology of resources (Luckin, 2008) framework situated within sociocultural learning theory (Lantolf \& Appel, 1994; Wang, 2003) allowed for a holistic exploration of teachers' educational context and the impact of the inter-related dimensions affecting teachers’ learning and use of laptops as instructional tools. Through this lens I combined teacher interviews and multiple classroom observations in order to identify the facilitative and barrier

factors affecting teacher instructional use of laptops in their high school science classrooms. The goal of this study was to help further understanding of how school district leaders can help teachers reach and engage students within their digital environment. The findings indicate several facilitating factors as well as some barrier factors affecting teacher instructional use of the laptops. This chapter presents discussion of the findings in relation to the relevant literature, conclusions, and implications for further research and for practice. To help frame this discussion, the major conclusions of this study include laptops enhance curriculum implementation, professional learning teams facilitate adult learning and use of the laptops, and successful use of laptops requires a community effort.

\section{Discussion}

This research was framed by major concepts from sociocultural learning theory and the ecology of resources framework, building from concepts from the diffusion of innovation and 
adult learning literature. Placing the teachers at the center of their educational context as adult learners allowed for a deeper understanding of the dynamic relationship among the factors and filters affecting technology use and teacher learning. Findings indicate several facilitating factors affecting instructional technology use and teacher learning as well as some factors hindering these goals.

\section{Facilitating Factors}

There are several factors the high school science teachers identified as facilitating their learning and instructional use of the laptops. I discuss these factors within the following categories: (1) the role of professional learning teams, (2) the value of peer relationships, (3) technology as a tool for learning, (4) the educational context, and (5) the role of explicit expectations.

Professional learning teams (PLTs). Literature concludes adult learning should be active, collaborative, and relevant (Cranton, 1994; Even, 1987; Knowles, 1970; Mezirow, 1991). The level of motivation for adult learners varies (Guzey \& Roehrig, 2012), with adults primarily motivated to learn in order to improve job performance (Beder, 1985; York-Barr, Sommers, Ghere, Montie, 2006). Consistent with the research, the high school science teachers reported their main motivation for learning is to improve instruction and learning for their students.

The teachers also reported they learn best through active practice and collaboration while differing in their level of motivation to learn about technology. The findings show teachers who are motivated to learn about technology independently can teach their peers who are less inclined to learn on their own time. The use of professional learning team time (PLT), also known as professional learning community (PLC) time, facilitates valuable collaboration among teachers. The PLT time helped to alleviate extra time outside the work day required by teachers to learn 
technology. This value cannot be over stated as time is a precious commodity in education settings (Cuban, 1986; York-Barr et al., 2006). Findings show the high school science teachers greatly value their PLTs. As teacher six shared, "PLT time has now become very sacred..." and teacher five said, “I want to learn from people who've been in the classroom recently, know what they're talking about, deal with real teenagers.”

Teacher learning and professional growth needs to be individual in nature (Clarke \& Hollingsworth, 2002). Findings from this study showed the teachers have different learning preferences and we must respect the role of their experience in relation to their professional learning. The literature also shows adults need to immediately apply their learning (Beder, 1985). Findings from this study demonstrated how teacher PLT time allows for the teachers to immediately apply what they learn from their peers regarding instructional use of the laptops. The teachers overwhelmingly reported highly valuing their PLT time because it was directly related to their planning for instruction while they were learning strategies from their peers for using the laptops instructionally and for overcoming barriers they encountered.

Value of peer relationships. Research literature finds social support and social, purposeful peer interactions are conducive to adult learning, including peer coaching (Ertmer \& Ottenbreit-Leftwich, 2010; Fullan, 2008; Levin \& Wadmany, 2008; Zhao \& Frank, 2003). Findings from this study collaborate, showing the teachers prefer to learn from their colleagues who are teaching within the same setting as they are. This desire showed to be especially strong for teachers more reluctant to use technology, as they shared the value of learning from their peers who demonstrate more interest in learning about technology outside the work day. They appreciate the tips and strategies their fellow teachers provide, and the overall support from their peers. 
Findings from this study also showed teachers in the PLTs combine their strengths and areas of interest in order to teach and support each other. These findings are consistent with the social nature of learning and sociocultural learning theory (Lantolf \& Appel, 1994; Wang, 2003), especially related to the role of the PLT or PLC, which fosters peer collaboration and continuous learning (Evans, 1996; Marquardt, 2011) vital to successful adult learning and change.

The high school science teachers reported their collaboration has facilitated successful use of the laptops in their classrooms during the second year of implementation. The findings also showed teachers' collaboration resulted in more timely improvements to instruction. The PLT time, combined with the high school's block schedule of extended periods, facilitate collaboration and professional learning. Teachers also reported the districtwide professional development (PD) opportunities enable collaboration across departments which typically do not interact together. Also, incentives for attendance at district PD outside the work day are important, especially because the findings show not all teachers are willing to dedicate time outside the school day for their professional learning. Consistent with adult learning literature, adult learning must be voluntary in terms of participation and acquisition (Knowles, 1970; Wolcott, 1977) in order for adults to be open to learning. When learning is forced, it is much less likely to result in positive learning outcomes.

Teachers appreciate their colleagues who are motivated to learn about technology voluntarily on their own time or by attending district professional development sessions. The district wide PD is also important because the teachers reported the PLTs meet within content areas, but not across content areas, although the teachers expressed an interest in doing so, but not at the expense of their collaboration time within their curricular area. 
Technology as a tool for learning. Research finds technology must be viewed by teachers as curriculum embedded, rather than as an add-on to instruction for most effective use (Hutchison \& Reinking, 2011). The participating teachers reported the laptops are valuable tools for implementing the Next Generation Science Standards (NGSS), recently adopted by the school district as part of their science curriculum. The teachers also reported the laptops support the modeling approach to instruction which is inquiry based and student centered and has shown positive student learning results from the American Chemical Society (see Table 1). Both of these priorities proved to be primary facilitating factors for the teachers' instructional use of the laptops.

The teachers also reported the laptops have improved the delivery of instruction, making it more active as well as providing for improved access to technology devices, and in turn, information. Research concludes technology tools enrich learning experiences by facilitating critical thinking, inquiry and discovery (Diem, 2010; Fullan, 2012). The teachers reported needing multiple tools to provide active, relevant, inquiry based instruction, including both print and digital resources. The teachers said education leaders should not replace print resources with electronic ones, but rather they should adjust the quantities to reflect the growing importance and reliance upon digital resources. Additionally, the teachers indicated students also have individual preferences for using print or electronic resources, and specific device types, depending on the subject matter and individual comfort level. Likewise, the teachers shared great interest and opinions about the types of devices and tools available to them, and they wanted their opinions valued by educational leaders. This finding aligns with those from Levin and Wadmany (2008) who stress the importance of listening to teachers and valuing their 
opinion. They found teachers are the key players in changing the educational world, and valuing their opinions is a critical component to that effort. We need to pay attention to the conditions affecting the teachers' educational context.

As a tool for learning, research shows technology is redefining literacy (Diem, 2010) and requiring the need for information literacy (Hennessy, Ruthven, \& Brindley, 2005; McLeod \& Lehman, 2012). Findings from this study showed ready access to information is transforming the role of the teacher who is no longer the only source of information in the room, as well as the skills students need to successfully navigate and use information. The teachers reported the increased access to devices has enabled more relevant, timely access to information, while also bringing a new level of need for information literacy for both students and teachers within the education setting. While the science teachers reported having the knowledge and skill to use the laptops instructionally, not all of them felt entirely prepared to help students evaluate the authenticity of information.

The educational context. The diffusion of innovation literature shows the importance of systems for diffusion of innovations, including intentional linkages (Anthony, 2012; Fullan 2008; Fullan, 2012; Park \& Ertmer, 2008), with all networks acting in concert together (Evans, 1996). Anthony (2012) found instructional technology efforts often exist outside district or school wide improvement efforts, leading to contradictory system linkages. In this case study's findings, the science teachers' PLTs are serving as systems for diffusing laptop use among the teachers in the science department despite the lack of explicit expectations from their school leaders. The teachers expressed the need for collaboration outside their department as well, but there is limited time or opportunity to do so. For example, science teachers could collaborate 
with language arts teachers or media specialists in order to further develop their information literacy skills. There is no system to make this collaboration possible and cooperation among departments appears to be fragmented.

Research literature identifies informal help and cooperative associations are valuable to teacher learning and collaboration in schools (Li, 2010; Zhao \& Frank, 2003). Additionally, Schon’s (1971) findings emphasize the role of formal and informal networks. Li (2010) also found informal networks instrumental in facilitating teacher receptiveness to innovations. Findings from this study show teachers report positive support within their educational setting, including technical staff, fellow science teachers, and students. Additionally, research illustrates the importance of a collegial environment and culture for helping teachers use technology in the classroom (Finger \& Houguet, 2009; Friedrich \& Hron, 2011; Levin \& Wadmany, 2008). The teachers in this study reported their school culture promotes a shared sense of responsibility and expectations for student success, including having positive relationships with technical staff.

The role of explicit expectations. Park and Ertmer (2008) found a lack of explicit expectations was a barrier to technology use. Teachers participating in this case study reported experiencing implicit expectations regarding laptop use rather than explicit expectations. It is surprising the teachers reported finding the implicit expectations sufficient, and in some ways appreciated. They appreciate the encouragement and respect they have received from district and building leaders. Teachers reported their leaders set forth the explicit expectation the teachers will engage students and produce successful learning results. They appreciate what they viewed as respect for their professional judgment by being given a great deal of latitude in their instructional pedagogy. 
However, many of the teachers expressed frustration with one other teacher's refusal to participate in a PLT, despite their indicating, "The peer pressure is there.” (Teacher 4) It may be the lack of explicit expectations is what permits this teacher to decline participation in a PLT, despite the clear frustration of the science teachers participating in this study. Teacher one has no other teacher to collaborate with within these teachers' content area due to the one teacher's resistance. As teacher one said, "He does his thing, and I do my thing.” This separation clearly presents a barrier to effective collaboration within this content area of the science department.

\section{Barrier Factors}

There are some factors the high school science teachers identified as barriers to their learning and instructional use of the laptops. I discuss these factors within the following categories: the digital divide, and issues of connectivity and compatibility.

The digital divide. Literature shows the digital divide results in equity issues for students as well as their parents (Diem, 2010; Machado-Casas \& Ruiz, 2012). When parents do not understand students' use of technology tools for learning, the technology can become a communication barrier, creating a “multigenerational rift” (Machado-Casas \& Ruiz, 2012). Likewise, students who do not have technology devices at home with which to practice can show a lack of skill at school. Teacher five shared, “I don't want those kids not to be able to rise to the same level as others.”

In this study, the teachers reported needing to be careful about assigning work requiring access to technology resources from home. The lack of student access from home to the Internet challenges assignments outside class. Additionally, student lack of access to devices while at 
home presents an equity issue, the teachers report. However, technology tools have the potential to bridge these digital divides, if education leaders continue to explore creative methods for accomplishing this goal (Machado-Casas \& Ruiz, 2012).

Issues of connectivity and compatibility. Findings in this study showed technical issues of device and software compatibility exist for the science teachers. The high school science teachers expressed a great deal of concern their special software and probes work on their laptops. The constant changes and updates to software versions present challenges for the teachers. Consistent with the technology literature, technology is evolving at an exponential pace (Best \& Kellner, 2001; Fullan, 2012; McLeod \& Lehmann, 2012). Therefore, it will continue to present compatibility challenges for teachers and technical staff members.

Also wireless connectivity and long computer login times present challenging barriers to instructional laptop use. However, during classroom observations, the teachers demonstrated knowing how to have alternate plans when technology issues present barriers. This finding was collaborated by the teacher interviews, and is consistent with findings of Levin and Wadmany (2008). Through their three year study, they concluded issues of connectivity and compatibility will exist, and teachers need to be willing and confident to overcome these challenges (2008).

Collaborative planning, sharing and learning among teachers within content areas, as well as regular feedback from team members, has allowed the teachers to improve their instruction in a timelier manner than before. When peers teach each other, sharing relevant strategies, successes, and failures, they can quickly adjust their lesson plans, leading to improved instruction. 


\section{Conclusions}

This case study was designed to answer two research questions: (1) How did the implementation of laptops in one high school's science classrooms affect teacher instructional practices, and (2) What factors did teachers identify as facilitating or hindering their learning and instructional use of laptops in high school science classrooms? A wide variety of findings have come from the data collected for this research, leading to three main ideas which can provide helpful information about teacher learning and their use of instructional technology: 1) laptops enhance curriculum implementation, 2) professional learning teams facilitate adult learning and use of the laptops, and 3) successful use of laptops requires a community effort.

During the second year of laptop use, teachers endorsed the laptops as valuable tools for implementing both the Next Generation Science Standards and the modeling approach to instruction adopted by many of the participating teachers. The laptops were viewed as tools to accomplish learning objectives, rather than add-ons to what teachers needed to do. Additionally, they helped the teachers engage students, who teacher one described as, "technology babies who are almost born with a device and a screen.” To allow for active, inquiry based learning, multiple tools are required and the teachers emphasized they need a combination of teacherapproved print and digital resources. Sometimes having students share devices is sufficient, other times students need their own device, depending on the learning objective. If we give teachers the right tools to meet curricular goals, which work when teachers need them to work, and time to plan, collaborate and experiment together, technology enhanced instruction can provide valuable benefits for student learning, provided teachers are knowledgeable about how to use the devices. 
Probably the most important take-away from examining teacher perspectives about using laptops was the vital role their professional learning teams (PLTs) played in helping teachers learn how to successfully use the laptops. This study took place during the course of a regular school year, when no extra resources were available to provide training for using the laptops instructionally. During the second year of implementation, the teachers reported having the knowledge and skills to use the laptops with their students because of the time they spent collaborating, planning and learning from each other on a regular basis. PLTs, also known as professional learning communities (PLCs), provide regular, on-going opportunities for teachers to break out of the isolation often found in teaching within their own separate classrooms (Johnson, 1990; Schon, 1983). Regular PLT time has reduced the barriers to teacher learning, collaboration, and reflective practice. Teachers reported regularly discussing substantive issues related to curriculum, their lessons, and laptop use during their PLT time, fostering ongoing collaboration and learning.

Interestingly, teachers who more reluctantly use technology tools with their students were observed using them after they learned strategies from their more technology adventurous peers. Upon collaborating with their peers, they were able to explore and investigate ways to better engage students, using the laptops as tools. The PLT time provides teachers time to collaborate as professional learners and contributors to teams who work to combine and share their strengths.

A shared sense of responsibility for supporting teachers is required from all areas of school districts. This study found in order to successfully use laptops, or other technology tools, multiple people and multiple tools are required for optimal results. It requires the entire education community working in a cohesive, systemic manner, with the right curriculum, tools 
and support systems in place. Teachers reported receiving good support from colleagues, technical staff, and their students, who are much more versed in the use of technology than many teachers; however, the science teachers also expressed a need for additional collaboration and support from those outside their science department. District departments used to working in isolation must integrate their support efforts.

Barriers related to infrastructure and device and software compatibility continue to exist for teachers. School districts must work diligently to minimize those barriers, trying to keep pace with the rapid changes occurring in the technology field (Brynjolfsson \& McAfee, 2011). The work is complicated and wrought with on-going challenges requiring the willingness to overcome and work through the barriers. Additionally, consistent expectations from the school community for the use of technology tools are necessary. The teachers reported interpreting the provision of the laptops and other technology tools as the expectation they be utilized to support the explicit expectation they engage and produce successful learning results for their students.

Our schools can benefit from a holistic, ecological approach to supporting teachers' use of technology. It requires complex planning to make all pieces function effectively as a unit, including curriculum, technology, and operations, especially because school districts often operate in isolated units (Schon, 1983). Research documents the need for educational leaders to understand the inter-related resource elements and influences affecting the educational context (Guzey \& Roehrig, 2012; Lin, 2011; Luckin, 2010; Luckin et al., 2012; Zhao \& Frank, 2003). Anthony conceptualizes technology implementation as a network of planning and implementation activities carried out by the school community. This study showed the teachers 
feel excellent support from multiple individuals, including students. However, there is evidence the teachers feel a separation from their building leaders in terms of the leaders' level of knowledge, involvement, support, and expectations regarding instructional technology.

The school district's leaders reported observing a wide range of use during the first year of the laptop implementation. Thanks to the peer network formed through the PLTs, use of the laptops has spread to include all of the participating teachers, yet, evidence shows at least one teacher remains outside the peer network. Until the entire community effort is cohesively dedicated to supporting and encouraging laptop use, there will be teachers who do not participate in the shared vision. While the laptops have proven to be valuable tools for implementing the science curriculum at this large Midwestern high school, and professional learning teams have proven to provide optimal learning opportunities facilitating collaboration and reflective learning among teachers, barriers related to people and technology challenges continue to exist.

\section{Implications for Future Research and Practice}

This study contributes to the current literature regarding teacher perspectives about using laptops as instructional tools, as well as how teachers perceive of themselves as adult learners. This single case study design using a holistic framework, allowed the researcher to understand and describe teacher views from a more nuanced perspective, with the teachers situated as learners at the center of their educational context.

However, this study's findings and conclusions suggest opportunities for further areas of research. There is a need to further explore the role of PLTs as a way to facilitate teacher learning to use technology instructionally and investigate how effectively PLTs are being used in schools, especially as a way for delivering professional development to teachers. There is also a need to further explore the role of implicit versus explicit expectations in regards to teachers 
using technology as a tool. For instance, is having the expectation students will be engaged in active, inquiry based instruction a sufficient expectation for teachers to use technology tools instructionally?

As students are demonstrating the ability to help support technology use in the classroom, we need to develop a better understanding of how teachers view their changing role in the classroom as they are no longer the only expert in the room. It could be valuable to explore what teachers can learn from students who are so much more in tune with technology than teachers may be. How can their knowledge about technology apply to the academic setting? Also, it is important to hear from students in relation to their perceptions of having teachers who approach instruction from a student-centered orientation versus a teacher directed approach, allowing researchers to compare the modeling approach to the traditional approach, which was described by one teacher as a, "pour it down your throat" method. (Teacher 4)

Additionally, within the science discipline, we should investigate how technology tools can best facilitate the implementation of the Next Generation Science Standards. This study did not evaluate technology use by the teachers as it only sought to understand teacher perspectives about its use. Also, as schools increase their reliance on technology tools and digital resources to support curriculum, there is a need to explore how schools are addressing issues related to the digital divide resulting from the increased reliance on digital resources not available to all students at home, and to the parents who are supporting those students.

Since supporting technology efforts is a complex endeavor requiring integrated support systems, we must explore how school districts can establish integrated systems of people and 
infrastructure to fully support the use of technology tools for enhancing instruction. Case studies could be conducted investigating school districts who have achieved system integration among their departments and support processes.

While this study investigated only one large Midwestern high school, the insights gained regarding how laptops have affected instructional practices, and the advice from the high school science teachers regarding factors enhancing or hindering their use of the laptops may be applicable to a variety of school settings. This study can offer some helpful implications for practice in order to facilitate the use of instructional technology tools and to reduce barriers to their use.

PLTs can be used to help teachers develop the knowledge and skills needed for using technology devices instructionally. Through the use of PLTs, learning for teachers becomes job embedded, timely, and relevant to improving their professional performance. Building leaders need to purposefully hire and assign teachers to PLTs, making sure they include teachers who prefer to learn about technology on their own time as part of each PLT. They can also provide increased direct district level support to the PLTs, especially to those who do not have teachers interested in learning about technology outside the work day. Also, technical staff and education leaders should consider providing professional development via PLTs in addition to offering district wide sessions. Educational leaders cannot ignore teachers’ strong preference for learning not only from other educators, but from their peers with whom they are currently teaching.

Education leaders need to set expectations regarding participation in PLTs, requiring it for all teachers, especially within departments. Also, these leaders need to communicate a shared vision, including expectations for how curricular goals will be achieved using technology tools. 
They must insure they adopt curriculum requiring active, inquiry based learning and encourage instructional approaches similar to the modeling approach so technology tools are used purposefully.

We must continue to provide technology devices as well as print resources and digital resources, but we can provide those in varying quantities with print materials serving a smaller, supporting role. Listening to teachers in order to fully understand what tools they need is important. Then educational leaders can best support teachers by providing those resources after hearing from the subject area experts.

Today's school district leaders must actively explore creative solutions for addressing the digital divide and provide equitable access to digital resources for students outside school. Additionally, resources are needed to address ongoing device and software compatibility issues inherent with technology tools' rapid advancements. Having dedicated IT staff at the school is helpful, but this support needs to be in addition to teachers and students working together to support each other. As teacher collaboration becomes the norm within departments, education leaders should explore ways to provide intentional cross-curricular PLT time for teachers. And finally, school district departments working in isolation must be strategically unified in order to provide integrated support systems working cohesively to support technology use in today's $21^{\text {st }}$ century classrooms. These lessons may be helpful to school leaders as they strive to understand how to facilitate the use of technology tools in which they have invested large sums of money.

\section{Summary}

In conclusion, this study examined factors facilitating and hindering instructional technology use in high school science classrooms. It also explored teacher perspectives about their own learning, and how use of the laptops affected their instructional practices. The 
participating teachers provided data through individual interviews and classroom observations. The findings of this case study suggest the use of PLTs can be valuable for facilitating teacher learning to use instructional technology tools. This study found PLTs to be a main facilitative factor for encouraging teacher learning and collaboration throughout the school year. Additionally, the whole school community, working as an integrated system rather than in isolation, is needed to most effectively support technology in education. Each school needs a building and district level community working in a coordinated effort in order for teachers to successfully use technology tools in support of educating students to be $21^{\text {st }}$ century learners. 


\section{Appendix A: Teacher Interview Protocol}

\section{Introduction}

Can you tell me a little bit about yourself? What courses do you teach? How long have you been teaching? How long at this school?

1. Please describe for me your experience with the additional laptops over the course of the past year and a half.

2. Think about the people you work with at your school and the school district. Describe how the People you interact with in your work encourage your use of the laptops with students and in what ways they may present obstacles.

3. Describe how the Knowledge and Skills you possess facilitate your use of the laptops with students and in what ways they may present obstacles.

4. Describe how the Tools you have available to you at work encourage your use of the laptops with students and in what ways they may present obstacles.

5. Describe ways your work Environment encourages your use of the laptops with students and in what ways it may present obstacles.

6. As a teacher, how do you learn best?

7. Please describe the values that guide your work as a teacher.

8. What expectations does your school leadership have for the use of the laptops? How about your curriculum coordinator?

9. How often do you collaborate with other teachers in your department? Within your school?

10. Some researchers say teachers don't use instructional technology because of legitimate concerns about it that outweigh the risk. What are your thoughts about that idea?

11. How would you describe your school culture?

12. In what ways have the additional laptops affected your instructional practices?

\section{Bringing the interview to a close:}

- Based on your experience, what advice do you have for other teachers who have additional technology tools in their classrooms? What about for school leaders?

- What questions or advice do you have for me about this research?

Thank you for participating in this interview. 


\section{Possible extra questions:}

1. What motivates you to try new instructional strategies?

2. How would you have responded if you had been given a choice about taking the laptops? What if you had to choose between the laptops or new text books?

3. How often do you look in on colleagues' classrooms, or do others look in on yours? How often do you discuss it afterwards?

4. How do you decide which tools and resources to use for teaching your curriculum content?

5. How does your school promote or encourage innovation in instructional practices?

6. How would you describe the level of trust among staff in your building? 


\section{Appendix B: Classroom Observation Guide}

Observation/Teacher:

Date/Time:

Class:

I. Classroom Observation Notes:

\section{Summary:}

\begin{tabular}{|l|l|}
\hline $\begin{array}{l}\text { People: What they are doing and how are they } \\
\text { interacting? }\end{array}$ & \\
\hline $\begin{array}{l}\text { Knowledge and Skills: Content being learned } \\
\text { and skills being demonstrated; type of } \\
\text { instruction being used: }\end{array}$ & \\
\hline $\begin{array}{l}\text { Tools: Tools being used? Tools available but } \\
\text { not in use? }\end{array}$ & \\
& \\
\hline $\begin{array}{l}\text { Environment: How is class organized? } \\
\text { Decorated? Managed? What is overall climate } \\
\text { of the class? Are any rituals observed? }\end{array}$ & \\
\end{tabular}

III. Follow up clarifying questions: 


\section{References}

American Educational Research Association. (2011). AERA Code of ethics. Retrieved from http://www.aera.net/Portals/38/docs/About_AERA/CodeOfEthics(1).pdf

Anthony, A. B. (2012). Activity theory as a framework for investigating district-classroom system interactions and their influences on technology integration. Journal of Research on Technology in Education, 44(4), 335-356.

Argyris, C. \& Schon, D. (1974). Theory in practice: Increasing professional effectiveness. San Francisco, CA: Jossey-Bass.

Avila, J., \& Moore, M. (2012). Critical literacy, digital literacies, and common core state standards: A workable union? Theory into Practice, 51(1), 27-33.

Beder, H. (1985). The relationship of knowledge sought to appropriate teacher behavior in adult education. Lifelong Learning, 9(1), 14-15.

Belland, B. R. (2009). Using the theory of habitus to move beyond the study of barriers to technology integration. Computers \& Education, 52(2), 353-364.doi:http://dx.doi.org/ 10.1016/j.compedu.2008.09.004

Best, S., \& Kellner, D. (2001). The postmodern adventure: Science, technology, and cultural studies at the third millennium. New York, NY: Guilford Press.

Betrus, A. (2012). Historical evolution of instructional technology in teacher education programs: A ten-year update. TechTrends, 56(5), 42-45.

Bielefeldt, T. (2012). Guidance for technology decisions from classroom observation. Journal of Research on Technology in Education, 44(3), 205-223.

Boles, S. R. (2011). Using technology in the classroom. Science Scope, 34(9), 39-43.

Bolman, L. G., \& Deal, T. E. (2008). Reframing organizations: Artistry, choice, and leadership (4th ed.). San Francisco, CA: Jossey-Bass.

Brown, A. L. (1992). Design experiments: Theoretical and methodological challenges in creating complex interventions in classroom settings. Journal of the Learning Sciences, 2(2), 141-178.

Bryant, M. T. (2004). The Portable Dissertation Advisor. Thousand Oaks, CA: Corwin Press.

Brynjolfsson, E., \& McAfee, A. (2011). Race against the machine: How the digital revolution is accelerating innovation, driving productivity, and irreversibly transforming employment and the economy. Lexington, MA: Digital Frontier Press, eBook. 
Caffarella, R. S. (2002). Planning programs for adult learners: A practical guide for educators, trainers, and staff developers (2nd ed.). San Francisco, CA: Jossey-Bass.

Center for Digital Education. (2013). Technology innovation in education. Retrieved from http://www.centerdigitaled.com/reports/

Cervero, R. M., \& Wilson, A. L. (1994). Planning as a process of negotiating interests. In R. M. Cervero \& A. L. Wilson. Planning responsibly for adult education: A guide to negotiating power and interests (pp. 13-32). San Francisco, CA: Jossey-Bass.

Christensen, C. M., Horn, M. B., \& Johnson, C. W. (2008). Disrupting class: How disruptive innovation will change the way the world learns. New York, NY: McGraw Hill.

Clarke, D., \& Hollingsworth, H. (2002). Elaborating a model of teacher professional growth. Teaching and Teacher Education, 18(8), 947-967.

Cohen, D. K. (1987). Educational technology, policy and practice. Educational Evaluation and Policy Analysis, 99(20), 153-170.

Cole, A.L. \& Knowles, J.G. (2000). Researching teaching: Exploring teacher development through reflexive inquiry. Needham Heights, MA: Allyn \& Bacon.

Cranton, P. (1994). Understanding and promoting transformative learning: A guide for educators of adults. San Francisco, CA: Jossey-Bass.

Creswell, J. W. (2009). Research design: Qualitative, quantitative, and mixed methods approaches ( $3^{\text {rd }}$ ed.). Thousand Oaks, CA: Sage.

Cuban, L. (1986). Teachers and machines: The classroom uses of technology since 1920. New York, NY: Teachers College Press.

Cuban, L. (2009). Oversold and underused: Computers in the classroom. Boston, MA: Harvard University Press.

Cuban, L., Kirkpatrick, H., \& Peck, C. (2001). High Access and Low Use of Technologies in High School Classrooms: Explaining an Apparent Paradox. American Educational Research Journal, 38(4), 813-834. doi:http://dx.doi.org.proxy.mul.missouri. edu/10.3102/00028312038004813

Dalton, B. (2013). Multimodal composition and the common core state standards. Reading Teacher, 66(4), 333-339.

Dani, D. E., \& Koenig, K. M. (2008). Technology and reform-based science education. Theory into Practice, 47(3), 204-211. 
Davenport, J. (1984). Adult educators and andragogical-pedagogical orientations: A review of the literature. Journal of Adult Education, 12(2), 9-17.

Deci, E. L. (1975). Intrinsic motivation. New York, NY: Plenum Press.

Deci, E. L., \& Ryan, R. M. (1985). Intrinsic motivation and self-determination in human behavior. New York, NY: Plenum Press.

Denzin, N. K. \& Lincoln, Y. S. (Eds.). (2011). The SAGE handbook of qualitative research. Thousand Oaks, CA: Sage.

Diem, R., \& Berson, M. J. (Eds.). (2010). Technology in retrospect: Social studies in the information age, 1984-2009. IAP.

Donnelly, D., McGarr, O., \& O’Reilly, J. (2011). A framework for teachers’ integration of ICT into their classroom practice. Computers \& Education, 57(2), 1469-1483.

Drew, S. V. (2013). Open up the ceiling on the common core state standards: Preparing students for 21st-century literacy--now. Journal of Adolescent \& Adult Literacy, 56(4), 321-330.

Emerson, R. M., Fretz, R. I., \& Shaw, L. L. (2011). Writing ethnographic fieldnotes. (2 ${ }^{\text {nd }}$ ed.). Chicago, IL: University of Chicago Press.

Ertmer, P. A. (1999). Addressing first- and second-order barriers to change: Strategies for technology integration. Educational Technology Research and Development, 47(4), 4761.

Ertmer, P. A. (2005). Teacher pedagogical beliefs: The final frontier in our quest for technology integration? Educational Technology Research and Development, 53(4), 25-40.

Ertmer, P. A., \& Ottenbreit-Leftwich, A. (2010). Teacher technology change: How knowledge, confidence, beliefs, and culture intersect. Journal of Research on Technology in Education, 42(3), 255-284.

Ertmer, P. A., Ottenbreit-Leftwich, A. T., Sadik, O., Sendurur, E., \& Sendurur, P. (2012). Teacher beliefs and technology integration practices: A critical relationship. Computers \& Education, 59(2), 423-435. doi:http://dx.doi.org.proxy.mul.missouri.edu/10.1016/j. compedu.2012.02.001

Evans, R. (1996). The human side of school change: Reform, resistance, and the real-life problems of innovation. San Francisco, CA: Jossey-Bass.

Even, M. J. (1987). Why adults learn in different ways. Lifelong Learning, 10(8), 22-25. 
Federal Communications Commission. (2013). Universal Service Program for Schools and Libraries. Retrieved from http://www.fcc.gov/guides/universal-service-program-schoolsand-libraries

Finger, G., \& Houguet, B. (2009). Insights into the intrinsic and extrinsic challenges for implementing technology education: Case studies of Queensland teachers. International Journal of Technology and Design Education, 19(3), 309-334.

Fink, A. (2009). How to conduct surveys: A step-by-step guide. (4th ed.). Thousand Oaks, CA: Sage.

Fleischer, H. (2012). What is our current understanding of one-to-one computer projects: A systematic narrative research review. Educational Research Review, 7(2), 107-122.

Friedman, A. M., \& Hicks, D. (2006). The state of the field: Technology, social studies, and teacher education. Contemporary Issues in Technology and Teacher Education, 6(2), 246248.

Friedrich, H. F., \& Hron, A. (2011). Factors affecting teachers' student-centered classroom computer use. Educational Media International, 48(4), 273-285.

Fullan, M. (2007). The new meaning of educational change (4th ed.). London, ENG: Teachers College Press.

Fullan, M. (2008). The six secrets of change: What the best leaders do to help their organizations survive and thrive. San Francisco, CA: Jossey-Bass.

Fullan, M. (2012). Stratosphere: Integrating technology, pedagogy, and change knowledge. Don Mills, CA: Pearson Canada.

Galvan, J. L. (2012). Writing literature reviews: A guide for students of the social and behavioral sciences (5th ed.). Glendale, CA: Pyrczak.

Garcia, A. (2012). Trust and mobile media use in schools. Educational Forum, 76(4), 430-433.

Gray, L., Thomas, N., \& Lewis, L. (2010). Teachers' use of educational technology in U.S. public schools: 2009. First Look. NCES 2010-040, 70.

Grix, J. (2004). The foundations of research. New York, NY: Palgrave.

Guba, E. G. (Ed.). (1990). The paradigm dialogue. Newbury Park, CA: Sage.

Guzey, S. S., \& Roehrig, G. H. (2012). Integrating educational technology into the secondary science teaching. Contemporary Issues in Technology and Teacher Education (CITE Journal), 12(2), 162-184. 
Hall, G. E. (2013). Evaluating change processes: Assessing extent of implementation (Constructs, methods and implications). Journal of Educational Administration, 51(3), 264-289.

Hall, G. E., \& Hord, S. M. (2011). Implementing change: Patterns, principles, and potholes. (3 ${ }^{\text {rd }}$ ed.). Upper Saddle River, NJ: Pearson.

Harris, J. B., \& Hofer, M. J. (2011). Technological pedagogical content knowledge (TPACK) in action: A descriptive study of secondary teachers' curriculum-based, technologyrelated instructional planning. Journal of Research on Technology in Education, 43(3), 211-229.

Harris, J., Mishra, P., \& Koehler, M. (2009). Teachers' technological pedagogical content knowledge and learning activity types: Curriculum-based technology integration reframed. Journal of Research on Technology in Education, 41(4), 393-416.

Hatch, J. (2002). Doing qualitative research in education settings. Albany, NY: State University of New York Press.

Hattie, J. (2009). Visible learning for teachers: A synthesis of over 800 meta-analyses relating to achievement. London, England: Routledge.

Hedberg, J. G. (2011). Towards a disruptive pedagogy: Changing classroom practice with technologies and digital content. Educational Media International, 48(1), 1-16. doi:10.1080/09523987.2011.549673

Hennessy, S., Ruthven, K., \& Brindley, S. (2005). Teacher perspectives on integrating ICT into subject teaching: Commitment, constraints, caution, and change. Journal of Curriculum Studies, 37(2), 155-192.

Hew, K. F., \& Brush, T. (2007). Integrating technology into K-12 teaching and learning: Current knowledge gaps and recommendations for future research. Educational Technology Research Development, 55, 223-252.

Holden, H., \& Rada, R. (2011). Understanding the influence of perceived usability and technology self-efficacy on teachers' technology acceptance. Journal of Research on Technology in Education, 43(4), 343-367.

Howard, S. K. (2011). Affect and acceptability: Exploring teachers’ technology-related risk perceptions. Educational Media International, 48(4), 261-272.

Hoy, W. K., Gage, C. Q., \& Tarter, J. C. (2006). School mindfulness and faculty trust: Necessary conditions for each other? Educational Administration Quarterly, 42(2), 236255.

Hsu, S. (2010). The relationship between teacher's technology integration ability and usage. Journal of Educational Computing Research, 43(3), 309-325. 
Hutchison, A., \& Reinking, D. (2011). Teachers’ perceptions of integrating information and communication technologies into literacy instruction: A national survey in the United States. Reading Research Quarterly, 46(4), 3-12-333. doi:10.1002/RRQ.002

International Society for Technology in Education (ISTE). (2008). National educational technology standards for teachers, 2nd edition. Eugene, OR: International Society for Technology in Education. Retrieved from http://www.iste.org/standards/ nets-for-teachers/nets-for-teachers-2008.aspx

Janis, I. L. (2011). Groupthink: The desperate drive for consensus at any cost. In J. M. Shafritz, J. S. Ott, \& Y. S. Jang (Eds.), Classics of organization theory ( $7^{\text {th }}$ ed., pp. 189-196). Boston, MA: Wadsworth. (Reprinted from Psychology today (5), pp. 43-76, 1971, New York, NY: Sussex)

John-Steiner, V., \& Mahn, H. (1996). Sociocultural approaches to learning and development: A Vygotskian framework. Educational Psychologist, 31(3/4), 1-17.

Johnson, D. (2012). Stretching your technology dollar. Educational Leadership, 69(4), 30-33.

Johnson, S. M. (1990). Teachers at work. New York, NY: Basic Books.

Jones, A., \& Moreland, J. (2004). Enhancing practicing primary school teachers’ pedagogical content knowledge in technology. International Journal of Technology and Design Education, 14(2), 121-140.

Jung, C. (1971). Psychological types. Princeton, NJ: Princeton University Press.

Keller, T. E., \& Pearson, G. (2012). A framework for K-12 science education: Increasing opportunities for student learning. Technology and Engineering Teacher, 71(5), 12-18.

Killian, J. E., \& Killian, R. L. (1983). Computer competency: Tailoring inservice to professional needs. Lifelong Learning, 7(1), 21-23.

Kitchenham, A. D. (2009). School cultures, teachers, and technology transformation. Canadian Journal of Learning and Technology, 35(2), 16.

Knowles, M. S. (1970). The modern practice of adult education: Andragogy versus pedagogy. New York, NY: Association Press.

Kopcha, T. J. (2010). A systems-based approach to technology integration using mentoring and communities of practice. Educational Technology Research and Development, 58(2), 175190. 
Kotrlik, J. W., \& Redmann, D. H. (2009). Analysis of teachers' adoption of technology for use in instruction in seven career and technical education programs. Career \& Technical Education Research, 34(1), 47-77.

Lajoie, S.P. (ed.). (2000). Computers as cognitive tools: No more walls, Vol. 2. Mahwah, NJ: Lawrence Erlbaum Associates.

Lantolf, J.P., \& Appel, G. (Eds.). (1994). Vygotskian approaches to second language research. Norwood, NJ: Ablex.

Lantolf, J. P. (2006). Sociocultural theory and L2: State of the art. Studies in Second Language Acquisition, 28(1), 67-109.

Lantolf, J. P., \& Beckett, T. G. (2009). Sociocultural theory and second language acquisition. Language Teaching, 42(4), 459-475. Retrieved from http://search.proquest.com/docview /61814172?accountid=14576

Lawless, K. A., \& Pellegrino, J. W. (2007). Professional development in integrating technology into teaching and learning: Knowns, unknowns, and ways to pursue better questions and answers. Review of Educational Research, 77(4), 575-614.

Lei, J., \& Zhao, Y. (2007). Technology uses and student achievement: A longitudinal study. Computers \& Education, 49(2), 284-296.

Levin, T., \& Wadmany, R. (2006). Teachers' beliefs and practices in technology-based classrooms: A developmental view. Journal of Research on Technology in Education, 39(2), 157-181.

Levin, T., \& Wadmany, R. (2008). Teachers' views on factors affecting effective integration of information technology in the classroom: Developmental scenery. Journal of Technology and Teacher Education, 16(2), 233-263.

Li, S. C. (2010). Social capital, empowerment and educational change: A scenario of permeation of one-to-one technology in school. Journal of Computer Assisted Learning, 26(4), 284295.

Lin, C. (2011). A learning ecology perspective: School systems sustaining art teaching with technology. Art Education, 64(4), 12-17.

Lincoln, Y. S., \& Guba, E. (1985). Naturalistic inquiry. Beverly Hills, CA: Sage.

Luckin, R. (2008). The learner centric ecology of resources: A framework for using technology to scaffold learning. Computers \& Education, 50(2), 449-462. doi:http://dx.doi.org/ 10.1016/j.compedu.2007.09.018 
Luckin, R. (2010). Learning contexts as ecologies of resources: A unifying approach to the interdisciplinary development of technology rich learning activities. International Journal On Advances in Life Sciences, 2(34), 154-164. Retrieved from http://www.thinkmind.org/index.php?view=article\&articleid=lifsci_v2_n34_2010_9

Luckin, R., Bligh, B., Manches, A., Ainsworth, S., Crook, C., \& Noss, R. (2012). Decoding learning: The proof, promise and potential of digital education. London, England: NESTA. Retrieved from http://www.nesta.org.uk/library/documents/DecodingLearningReport_ v12.pdf

Machado-Casas, M. \& Ruiz, E. (2012). Globalized students vs. unglobalized families: Limiting family participation in education. Association of Mexican-American Educators (AMAE) Journal, 6(2), 4-12.

Mahn, H., \& John-Steiner, V. (2002). The gift of confidence: A Vygotskian view of emotions. In G. Wells \& G. Claxton (Eds.), Learning for life in the 21st century: Sociocultural perspectives on the future of education. (pp. 46-58). Malden, MA: Blackwell Publishing.

Marquardt, M. J. (2011). Building the learning organization: Achieving strategic advantage through a commitment to learning ( $3^{\text {rd }}$ ed.). Boston, MA: Nicholas Brealey.

McLeod, S., \& Lehmann, C. (Eds.). (2012). What school leaders need to know about digital technologies. San Francisco, CA: Jossey-Bass.

Merriam, S. B. (1998). Qualitative research and case study applications in education. San Francisco, CA: Jossey-Bass.

Merriam, S. B. (2009). Qualitative research: A guide to design and implementation. San Francisco, CA: Jossey-Bass.

Mezirow, J. (1991). Transformative dimensions of adult learning. San Francisco, CA: JosseyBass.

Moeller, B., \& Reitzes, T. (2011). Integrating technology with student-centered learning. Quincy, MA: Nellie Mae Education Foundation.

Mueller, J., Wood, E., Willoughby, T., Ross, C., \& Specht, J. (2008). Identifying discriminating variables between teachers who fully integrate computers and teachers with limited integration. Computers \& Education, 51(4), 1523-1537. doi:http://dx.doi.org/10.1016 /j.compedu.2008.02.003

Park, S. H., \& Ertmer, P. A. (2008). Examining barriers in technology-enhanced problem-based learning: Using a performance support systems approach. British Journal of Educational Technology, 39(4), 631-643. doi:http://dx.doi.org.proxy.mul.missouri.edu/10.1111/j.14678535.2008.00858.x 
Petko, D. (2012). Teachers' pedagogical beliefs and their use of digital media in classrooms: Sharpening the focus of the 'will, skill, tool' model and integrating teachers' constructivist orientations. Computers \& Education, 58(4), 1351-1359.

Podeschi, R. L., \& Pearson, E. M. (1986). Knowles and Maslow: Differences about freedom. Lifelong Learning, 9(7), 16-18.

Ravitch, S. M., \& Riggan, M. (2012). Reason \& rigor. Thousand Oaks, CA: Sage.

Roberts, C. M. (2010). The dissertation journey: A practical and comprehensive guide to planning, writing, and defending your dissertation. $\left(2^{\text {nd }}\right.$ ed.). Thousand Oaks, CA: Corwin.

Rogers, E. M. (1962). Diffusion of innovations. New York, NY: The Free Press.

Sample, J. A., \& Kaufman, R. (1986). A holistic program development model for adult educators. Lifelong Learning, 9(4), 18-23.

Schein, E. H. (1985). Organizational culture and leadership: A dynamic view. San Francisco, CA: Jossey-Bass.

Schein, E. H. (1992). Organizational culture and leadership. ( $2^{\text {nd }}$ ed.). San Francisco, CA: Jossey-Bass.

Schmoker, M. (2006). Results now: How we can achieve unprecedented improvements in teaching and learning. Alexandria, VA: Association for Supervision and Curriculum Devleopment.

Schon, D. A. (1967). Invention and the evolution of ideas. London, England: Social Science Paperbacks.

Schon, D. A. (1971). Beyond the stable state. New York, NY: Random House.

Schon, D. A. (1983). The reflective practitioner: How professionals think in action. New York, NY: Basic Books.

Sheffield, C. (2011). Navigating access and maintaining established practice: Social studies teachers' technology integration at three Florida middle schools. Contemporary Issues in Technology and Teacher Education (CITE Journal), 11(3), 282-312.

Shieh, R. S. (2012). The impact of technology-enabled active learning (TEAL) implementation on student learning and teachers' teaching in a high school context. Computers \& Education, 59(2), 206-214. 
Shim, J. E., \& Li, Y. (2006). Applications of cognitive tools in the classroom. In M. Orey (Ed.), Emerging perspectives on learning, teaching, and technology. Retrieved from http://projects.coe.uga.edu/epltt/

Spires, H. A., Oliver, K., \& Corn, J. (2011). The new learning ecology of one-to-one computing environments: Preparing teachers for shifting dynamics and relationships. Journal of Digital Learning in Teacher Education, 28(2), 63-72.

Stake, R. E. (2010). Qualitative research: Studying how things work. New York, NY: The Guilford Press.

Taylor, F. W. (1911). The principles of scientific management. New York, NY: Harper and Brothers.

Tripp, T. R., \& Rich, P. J. (2012). The influence of video analysis on the process of teacher change. Teaching and Teacher Education, 28, 728-739.

Underwood, J., Smith, H., Luckin, R., \& Fitzpatrick, G. (2008). E-science in the classroom towards viability. Computers \& Education, 50(2), 535-546. doi:http://dx.doi.org/10.1016/ j.compedu.2007.07.003

Vannatta, R. A., \& Fordham, N. (2004). Teacher dispositions as predictors of classroom technology use. Journal of Research on Technology in Education, 36(3), 253-271.

Vygotsky, L. S. (1978). In Cole, John-Steiner, M., Scribner, S., \& Souberman, E. (Eds.) Mind in society: The development of higher psychological processes. Cambridge, MA: Harvard University Press.

Wang, H. (2003). Vygotsky and his theory. Journal of Educational Computing, Design, \& Telecommunication, 3(1). Retrieved from http://coe.ksu.edu/jecdol/Vol_3/

Weiss, R.S. (1994). Learning from strangers: The art and method of qualitative interview studies. New York, NY: The Free Press.

Wertsch, J. V. (1991). Voices of the mind: A sociocultural approach to mediated action. Cambridge, MA: Harvard University Press.

Wertsch, J. V. (1994). Mediated action in sociocultural studies. Mind, Culture, and Activity, 1(4), 202-208.

Wolcott, H. F. (1977). Teachers vs technocrats. Walnut Creek, CA: AltaMira Press.

Wolfinger, N. H. (2002). On writing fieldnotes: collection strategies and background expectancies. Qualitative Research, 2(1), 85-93. doi:10.1177/1468794102002001640 
Wu, W., Chang, H., \& Guo, C. (2008). An empirical assessment of science teachers' intentions toward technology integration. The Journal of Computers in Mathematics and Science Teaching, 27(4), 499-520.

Yin, R. K. (2014). Case study research: Design and methods. (5 ${ }^{\text {th }}$ ed.). Thousand Oaks, CA: Sage.

York-Barr, J., Sommers, W. A., Ghere, G. S., \& Montie, J. (2006). Reflective practice to improve schools: An action guide for educators. ( $2^{\text {nd }}$ ed.). Thousand Oaks, CA: Corwin.

Zhao, Y., \& Frank, K. A. (2003). Factors affecting technology uses in schools: An ecological perspective. American Educational Research Journal, 40(4), 807-840. 
Dr. Chris Diggs is a professional in the field of educational leadership. She received her doctor of philosophy degree in Educational Leadership and Policy Analysis from the University of Missouri-Columbia in December 2014. For the past 19 years she has worked for Columbia Public Schools, most recently as the Director of Technology Services, where she received the Outstanding Administrator Award for the 2013-2014 school year. Prior to her role as director, she worked as an Instructional Technology Coach, providing professional development to teachers. Previously, she worked as a Media Specialist, where she was named Outstanding Elementary Educator of the Year. Dr. Diggs also has experience in sales and management prior to her working in the education field. She received her Master’s degree in Information Science and Learning Technologies from the University of Missouri-Columbia, and her Bachelor's degree in Elementary Education from the University of Florida where she was recognized as an Outstanding Woman Leader. Dr. Diggs and her husband have five daughters and they reside in Columbia, Missouri. 